\title{
Pure spinor computation towards open string three-loop
}

\author{
I.Y. Park \\ Department of Physics, Kyoto University, Kyoto 606-8502, Japan \\ High Energy Accelerator Research Organization (KEK) \\ Tsukuba, Ibaraki 305-0801, Japan \\ Department of Natural and physical Sciences, Philander Smith College ${ }^{1}$ \\ Little Rock, AR 72223, USA \\ inyongpark05@gmail.com
}

\begin{abstract}
Using the recent results in the pure spinor formulation, we lay out a ground-work towards the full momentum space amplitudes of open superstrings at three-loop. After briefly reviewing the one-loop amplitude, we directly work out the two-loop amplitude and reproduce the result that was obtained by a symmetry argument before. For the three-loop, first we use the two-loop regulator as a warm-up exercise. The result vanishes. We then employ the regulator that has been recently proposed by Aisaka and Berkovits. It is noted that the terms in higher power in $\frac{1}{\lambda \lambda}$ that render the two-loop regulator disqualified for the three-loop do not contribute. This with a few other indications suggests a possibility that the AB regulator might also lead to a vanishing result. Nevertheless, we argue that it is possible to acquire the three-loop amplitude, and present a result that we anticipate to be the three-loop amplitude.
\end{abstract}

\footnotetext{
${ }^{1}$ Home institute
} 


\section{Introduction}

D-brane physics $[1,2,3]$ has brought many groundbreaking results in the recent developments in string theory and phenomenology. In particular, it has opened up a new route to understanding gravitational physics through gauge degrees of freedom $[4,5,6,7,8,9]$. At the heart of this phenomenon lies the fact that a D-brane admits two different descriptions, one as a flat hyperplane on which open strings move with their end points attached, and the other through a solitonic solution of closed string theory.

Most of the studies so far have been devoted to analyzing how the gauge/gravity correspondence works and discovering more examples. While those are meaningful tasks, an equally important task would be to understand why there is such a correspondence. Once unraveled, the underlying mechanism is likely to lead to a derivation from the first principle of the correspondence in its precise form. At the very least it will hint at a route to a derivation. The derivation is especially important since there are some outstanding questions. For example, are there circumstances where it is necessary to take into account the massive modes of an open string on top of the massless modes, the SYM? Another question is, does the conjecture remain valid in the non-planar regime?

A close interplay between an open string and a closed string must be responsible for the correspondence. ${ }^{1}$ We believe that the mechanism underlying the gauge/gravity duality should be sought [11] in the unity of degrees of freedom between an open string and a closed string $[12,13,14]$. If true, it would imply that the physics associated with an open string would be closely related to that of a closed string. A conjecture that may be viewed hinging on such an interplay was put forward in [15] and verified in the subsequent work [16] at one-loop. The two-loop extension was also discussed in [17]. There it was demonstrated that the open string loop divergences on a D-brane may be removed by a vertex operator constructed out of the curved geometry of the D-brane.

As part of the endeavor to extend the conjecture to the three-loop, we study the three-loop amplitudes of open strings. Unlike the one- and two-loop cases, no full momentum space amplitude has been computed at this level. Therefore it is necessary to compute the amplitudes themselves before attempting to extend the conjecture to the three-loop. A multi-loop computation in superstring theory is a complicated task. The complete two-loop amplitudes have been computed only fairly recently [18, 19]. Since the three-loop computations will be much more complicated, it is very important to use an effective method. To our view, the pure spinor formulation [20], which was developed by using some of the ingredients in [21], may provide such a tool. Also see $[22,23,24,25,26,27,28,29,30,31]$ for the further developments of the formulation. For example, compared with the RNS formulation, it requires far less amount of algebra

\footnotetext{
${ }^{1}$ In relation to this, we find the works of [10] interesting.
} 
to obtain the two-loop amplitude by direct computation as we show in section 3 below. ${ }^{2}$

For the three-loop order, first we use the two-loop regulator as a warm-up exercise. The result vanishes. We then employ the regulator that has been recently proposed by Aisaka and Berkovits (AB). It is noted in section 3 that the terms in higher power in $\frac{1}{\lambda \lambda}$ that render the two-loop regulator disqualified for the three-loop do not contribute. This with a few other indications suggests a possibility that the AB regulator might also lead to a vanishing result. This might be a signal of incompleteness of the AB regulator. ${ }^{3}$ To determine whether the entire three-loop indeed vanishes, all of the overall numerical coefficients of the individual terms must be kept track of. As will be fathomed in the subsequent sections, it is an extremely tedious task ${ }^{4}$ that may require some efficient method of organization. Our main goal is to determine the form of the three-loop amplitude, and we argue that it is possible to acquire the three-loop amplitude without using a precise and complete regulator. The basic idea is to rely on a pattern that the computations reveal. We make an observation on the pattern starting with the two-loop case below. Observed in a few chosen cases of the three-loop as well, the pattern is expected to persist in the higher loop orders.

The rest of the paper is organized as follows. In this paper, the four-point amplitude is analyzed. In the beginning of the next section, we briefly review the one-loop case to set the notations and convention. The two- and three- loop computations involve lengthy algebra. We present a summary of the results with a discussion of the pattern that plays an important role in determining the three-loop amplitude. In section 3 , we directly calculate the two-loop amplitude. The result is expressed in terms of the SYM multiplets and the covariant derivatives, thereby reproducing the result that was first obtained by a symmetry argument. The simplified measure introduced in [33] significantly reduces the amount of algebra that has be carried out. At three-loop a complete gauge invariant regulator is not currently available, at least not in a simple form. In section 4, we carry out our analysis using the regulator that has recently been proposed by Aisaka and Berkovits [34]. After carrying out [ds]- and $d d$ - integrations, we evaluate a few terms. We propose an expression, eq.(103), that we anticipate to be the correct three-loop amplitude. We end with future directions in the conclusion where few necessary checks and/or works are listed either to strengthen or to confirm our three-loop result.

\footnotetext{
${ }^{2}$ After we finished our computation, a work [32] had appeared that had some overlap with our two-loop computation.

${ }^{3}$ We believe that there is a room for a better understanding of a three-loop regulator. It deserves a separate study. We will further comment on this in the section 4 and the conclusion.

${ }^{4}$ In spite of the expected tediousness, tracking precise numerical coefficients for an amplitude in general should be an executable task. However, it would be more meaningful to carry out the task for a regulator that is free of any subtlety. The task is best left to future efforts after further progress on the regulator issue is made.
} 


\section{Summary of results}

Since the sections 3 and 4 on the loop-computations involve lengthy and tedious algebras, it may be useful to summarize the results before we embark on the computations. We start in section 2.1 by briefly reviewing the non-minimal formulation and the one-loop computation. This will set our notations and convention. In section 2.2, a summary of the two- and three- loop results is presented. There we ponder on the pattern that the two- and three- loop computations reveal. The three-loop amplitude, (25) (quoted from eq.(103) in section 4), has been obtained based on the pattern.

\section{1 review of non-minimal formalism and one-loop}

There are two versions of the pure spinor formulation:the minimal and the non-minimal. In this paper we use the non-minimal version. Our notations and some useful identities are summarized in Appendices A and B. We review several ingredients of the formulation that we will need in the calculations in sections 3 and 4 . For more details, we refer to $[24,30]$.

The unintegrated and the integrated vertex operators that represent the massless modes are respectively given by

$$
\begin{aligned}
V & =\lambda^{\alpha} A_{\alpha} \\
U & =\partial \theta^{\alpha} A_{\alpha}+\Pi^{m} A_{m}+d_{\alpha} W^{\alpha}+\frac{1}{2} N^{m n} \mathcal{F}_{m n}
\end{aligned}
$$

where

$$
\Pi^{m}=\partial X^{m}+\frac{1}{2} \theta^{\beta} \gamma^{m} \partial \theta
$$

The SYM multiplets satisfy

$$
\begin{aligned}
A_{m} & =\frac{1}{8} D_{\alpha} \gamma_{m}^{\alpha \beta} A_{\beta} \\
W^{\beta} & =\frac{1}{10} \gamma_{m}^{\alpha \beta}\left(D_{\alpha} A^{m}-\partial^{m} A_{\alpha}\right) \\
\mathcal{F}_{m n} & =\frac{1}{8} D_{\alpha}\left(\gamma_{m n}\right)^{\alpha}{ }_{\beta} W^{\beta}=\partial_{[m} A_{n]}
\end{aligned}
$$

and

$$
\begin{aligned}
& -D_{\alpha} A_{\beta}-D_{\beta} A_{\alpha}+\gamma_{\alpha \beta}^{m} A_{m}=0 \\
& D_{\alpha} A_{m}-\partial_{m} A_{\alpha}-\gamma_{m \alpha \beta} W^{\beta}=0 \\
& -D_{\alpha} W^{\beta}+\frac{1}{4}\left(\gamma^{m n}\right)_{\alpha}{ }^{\beta} \mathcal{F}_{m n}=0 \\
& \lambda^{\alpha} \lambda^{\beta}\left(\gamma_{m n}\right)_{\beta}{ }^{\gamma} D_{\alpha} \mathcal{F}^{m n}=0
\end{aligned}
$$


where the 16 by 16 gamma matrices, $\gamma_{m n}$, satisfy

$$
\eta_{m n} \gamma_{\alpha(\beta}^{m} \gamma_{\gamma \delta)}^{n}=0
$$

Using $D_{\alpha} \mathcal{F}^{m n}=2 k^{[m} \gamma_{\alpha \beta}^{n]} W^{\beta}$ and $-D_{\alpha} W^{\beta}+\frac{1}{4}\left(\gamma^{m n}\right)_{\alpha}{ }^{\beta} \mathcal{F}_{m n}=0$, one can show

$$
\begin{aligned}
& D_{\beta} D \alpha \mathcal{F}_{m n}=-\frac{1}{2} k_{[m}\left(\gamma_{n]} \gamma^{t u}\right)_{\alpha \beta} \mathcal{F}_{t u} \\
& D_{\delta} D_{\gamma} D_{\beta} D \alpha \mathcal{F}_{m n}=\frac{1}{4} k_{[m}\left(\gamma_{n]} \gamma^{t u}\right)_{\alpha \beta} k_{t}\left(\gamma_{u} \gamma^{v w}\right)_{\gamma \delta} \mathcal{F}_{v w}
\end{aligned}
$$

from which it follows

$$
\begin{aligned}
D_{\alpha_{1}} D_{\alpha_{2}} W^{\beta_{1}} & =\frac{1}{2} k^{m}\left(\gamma_{m n}\right)_{\alpha_{2}}{ }^{\beta_{1}}\left(\gamma^{n}\right)_{\alpha_{1} \gamma} W^{\gamma} \\
D_{\alpha_{1}} D_{\alpha_{2}} D_{\alpha_{3}} W_{(1)}^{\beta_{1}} & =-\frac{1}{8}\left(\gamma^{l_{1} l_{2}}\right)_{\alpha_{3}}{ }^{\beta_{1}} k_{l_{1}}^{(1)}\left(\gamma_{l_{2}} \gamma^{v_{1} v_{2}}\right)_{\alpha_{2} \alpha_{1}} \mathcal{F}_{v_{1} v_{2}}^{(1)}
\end{aligned}
$$

In an abelian case, one has

$$
\gamma_{\alpha \beta}^{m} \partial_{m} W^{\beta}=0
$$

It implies on shell

$$
\gamma_{\alpha \beta}^{m} k_{m} W^{\beta}=\not k W=0
$$

which is used in the two-loop computation in section 3. The pure spinor formulation provides algorithms for $N$-loop amplitudes. Up to and including the three-loop, they are given by

$$
\begin{aligned}
& \mathcal{A}_{1-\text { loop }}=\int d \tau<\mathcal{N}_{1}(y) \int d w \mu(w) b(w) V_{1}\left(z_{1}\right) \int d z_{2} U_{2}\left(z_{2}\right) \cdots \int d z_{N} U_{N}\left(z_{N}\right)> \\
& \mathcal{A}_{2-\text { loop }}=\int d \tau_{1} d \tau_{2} d \tau_{3}<\mathcal{N}_{2}(y) \prod_{s=1}^{3} \int d w_{s} \mu\left(w_{s}\right) b\left(w_{s}\right) \int d z_{1} U_{1}\left(z_{1}\right) \cdots \int d z_{N} U_{N}\left(z_{N}\right)> \\
& \mathcal{A}_{3-\text { loop }}=\int d \tau_{1} d \cdots d \tau_{6}<\mathcal{N}_{3}(y) \prod_{s=1}^{6} \int d w_{s} \mu\left(w_{s}\right) b\left(w_{s}\right) \int d z_{1} U_{1}\left(z_{1}\right) \cdots \int d z_{N} U_{N}\left(z_{N}\right)>
\end{aligned}
$$

where the ghost operator, $b$, is given by

$$
\begin{gathered}
b=s^{\alpha} \partial \bar{\lambda}_{\alpha}+\frac{\bar{\lambda}_{\alpha}\left[2 \Pi^{m}\left(\gamma_{m} d\right)^{\alpha}-N_{m n}\left(\gamma^{m n} \partial \theta\right)^{\alpha}-J_{\lambda} \partial \theta^{\alpha}-\partial^{2} \theta^{\alpha}\right]}{4 \bar{\lambda} \lambda} \\
+\frac{\left(\bar{\lambda} \gamma^{m n p} r\right)\left(d \gamma^{m n p} d+24 N_{m n} \Pi_{p}\right)}{192(\bar{\lambda} \lambda)^{2}}-\frac{\left(r \gamma_{m n p} r\right)\left(\bar{\lambda} \gamma^{m} d\right) N^{n p}}{16(\bar{\lambda} \lambda)^{3}}+\frac{\left(r \gamma_{m n p} r\right)\left(\bar{\lambda} \gamma^{p q r} r\right) N^{m n} N_{q r}}{128(\bar{\lambda} \lambda)^{4}}
\end{gathered}
$$


The $\mathcal{N}_{g},(g=1,2,3)$ in $(10)$ is the $g$-loop regulator. Each field has different number of zero modes: for the bosonic fiilds,

$$
\begin{array}{ccccc}
x^{m} & \lambda^{\alpha} & w_{\alpha} & \bar{\lambda}_{\alpha} & \bar{w}^{\alpha} \\
10 & 11 & 11 g & 11 & 11 g
\end{array}
$$

and for the fermionic fields,

$$
\begin{array}{cccc}
\theta^{\alpha} & d_{\alpha} & r & s \\
16 & 16 g & 11 & 11 g
\end{array}
$$

For the two- and the three- loop amplitudes, we will make frequent use of the 32component Fierz rearrangement lemma, which is reviewed in Appendix B. Because of this, we embed the 16-component notation in the 32-component notation. Let us define

$$
\lambda_{u}=\left(\begin{array}{c}
\lambda^{\alpha} \\
0
\end{array}\right), \quad \lambda_{d}=\left(\begin{array}{c}
0 \\
\lambda^{\alpha}
\end{array}\right)
$$

and similarly for other fields. Our conventions are summarized in Appendix A. The 32 by 32 gamma matrices are

$$
\Gamma^{m}=\left(\begin{array}{cc}
0 & \left(\gamma^{m}\right)_{\alpha \beta} \\
\left(\gamma^{m}\right)^{\alpha \beta} & 0
\end{array}\right),
$$

The 16-component relation

$$
\left(\lambda \gamma^{m} \ldots\right)\left(\ldots \gamma_{m} \lambda\right)=0
$$

translates into the 32-component relation

$$
\left(\lambda_{u} \Gamma^{m} \ldots\right)\left(\ldots \Gamma_{m} \lambda_{d}\right)=0
$$

The following combination of $\lambda$-fields appears as a part of the $[d s]$-integration measure

$$
\left(\lambda \gamma_{m}\right)_{\kappa_{1}}\left(\lambda \gamma_{n}\right)_{\kappa_{2}}\left(\lambda \gamma_{p}\right)_{\kappa_{3}} \gamma_{\kappa_{4} \kappa_{5}}^{m n p}: \text { anti-symmetric in } \kappa^{\prime} \mathrm{S}
$$

It is is totally antisymmetric in the $\kappa$-indices: as we will see, it provides a very powerful freedom in various index contractions. Throughout the computations we do not precisely record the overall numerical coefficients and some of the irrelevant non-numerical factors. ${ }^{5}$

\footnotetext{
${ }^{5}$ Not recording precise coefficients is a common practice in the pure spinor literature. In a high order loop computation such as two- or (especially) three- loop, there are many terms that need to be computed term by term. Many of these terms actually vanish. Therefore it is important first to efficiently identify the terms that lead to finite results. In addition, we suspect that there is some subtlety in the $\mathrm{AB}$ regulator. The upshot of section 4, can be put that one may obtain the three-loop amplitude by deliberately not tracking the accurate coefficients. This is because the AB regulator might produce a vanishing amplitude due to pair-wise cancelations. Eventually one should include not only the numerical coefficients but also the functions of moduli parameters. For the two-loop, this was done in a recent paper by Gomez and Mafra [32].
} 
The original one-loop computation was relatively simple. What makes it even simpler is the introduction of new measures for, $[d r]$ and $[d s]$ in [33],

$$
\begin{aligned}
& {[d r] } \doteq\left(\bar{\lambda} \gamma^{m}\right)^{\alpha_{1}}\left(\bar{\lambda} \gamma^{n}\right)^{\alpha_{2}}\left(\bar{\lambda} \gamma^{p}\right)^{\alpha_{3}}\left(\gamma_{m n p}\right)^{\alpha_{4} \alpha_{5}} \epsilon_{\alpha_{1} \ldots \alpha_{5} \delta_{1} \ldots \delta_{11}} \partial_{r}^{\delta_{1}} \cdots \partial_{r}^{\delta_{11}} \\
& {[d s] \doteq \frac{1}{(\lambda \bar{\lambda})^{3}}\left(\lambda \gamma^{m}\right)_{\alpha_{1}}\left(\lambda \gamma^{n}\right)_{\alpha_{2}}\left(\lambda \gamma^{p}\right)_{\alpha_{3}}\left(\gamma_{m n p}\right)_{\alpha_{4} \alpha_{5}} \epsilon^{\alpha_{1} \ldots \alpha_{5} \delta_{1} \ldots \delta_{11}} \partial_{\delta_{1}}^{s} \cdots \partial_{\delta_{11}}^{s} }
\end{aligned}
$$

These measures reduce to a great extent the amount of the algebra involved in the twoand three- loops. For one- and two- loops, we use the regulator given in [30]

$$
\mathcal{N}_{2}=e^{-\bar{\lambda} \lambda-r \theta-\bar{w} w+s d}
$$

instead of the original regulator. Unlike the latter, the former is not gauge invariant. ${ }^{6}$ For our purpose, it is only necessary to evaluate the $\left[d s^{I}\right]$ - and $\left[d d^{I}\right]$ - integrations. The one-loop analysis goes as follows. Saturating the 16 zero modes, the one-loop amplitude contains

$$
\begin{aligned}
\mathcal{K}= & \int[d \lambda][d \bar{\lambda}][d r][d \theta][d w][d \bar{w}][d s][d d] e^{-\bar{\lambda} \lambda-r \theta-\bar{w} w+s d} \\
& \frac{\left(\bar{\lambda} \gamma^{m n p} r\right)\left(d \gamma_{m n p} d\right)}{192(\bar{\lambda} \lambda)^{2}}\left[\lambda^{\alpha_{1}} A_{\alpha_{1}}^{(1)}\right]\left[d_{\alpha_{2}} W_{(2)}^{\alpha_{2}}\right]\left[d_{\alpha_{3}} W_{(3)}^{\alpha_{3}}\right]\left[d_{\alpha_{4}} W_{(4)}^{\alpha_{4}}\right]
\end{aligned}
$$

Carrying out $[d s]$ and $[d d]$ integration leads to (up to an overall numerical factor)

$$
\begin{aligned}
\mathcal{K} \doteq & \int[d \lambda][d \bar{\lambda}][d \theta][d w][d \bar{w}] e^{-\bar{\lambda} \lambda-\bar{w} w}\left(\bar{\lambda} \gamma^{r}\right)^{\alpha_{1}}\left(\bar{\lambda} \gamma^{s}\right)^{\alpha_{2}}\left(\bar{\lambda} \gamma^{t}\right)^{\alpha_{3}}\left(\gamma_{r s t}\right)^{\alpha_{4} \alpha_{5}} \epsilon_{\alpha_{1} \ldots \alpha_{5} \delta_{1} \ldots \delta_{11}} \theta^{\delta_{1}} \ldots \theta^{\delta_{11}} \\
& \frac{\left(\bar{\lambda} \gamma^{m n p} D\right)}{(\bar{\lambda} \lambda)^{5}}\left[\lambda A_{(1)}\right]\left[\lambda \gamma^{m} W_{(2)}\right]\left[\lambda \gamma^{n} W_{(3)}\right]\left[\lambda \gamma^{p} W_{(4)}\right]
\end{aligned}
$$

which can be further evaluated to get the momentum space expression, often called $K_{0}$, in the literature.

\section{2 two- and three- loop results}

The two- and three- loop computations follow similar, although more complex, steps. After saturating the $d$-zero modes, one gets two- and three- loop analogues of (19). While the one-loop expression, (19), has the factor of $\left(d \gamma_{m n p} d\right)$, the corresponding twoand three- loop expressions contain $\left(d \gamma_{m n p} d\right)^{3}$ and $\left(d \gamma_{m n p} d\right)^{5}$ (or $\left.\left(d \gamma_{m n p} d\right)^{6}\right)$ respectively in a schematic notation. The zero mode parts of these factors are

$$
\left(d \gamma_{m n p} d\right) \doteq \quad\left(d_{0} \gamma_{m n p} d_{0}\right) \oplus\left(d_{0}^{\prime} \gamma_{m n p} d_{0}^{\prime}\right) \oplus\left(d_{0} \gamma_{m n p} d_{0}^{\prime}\right)
$$

\footnotetext{
${ }^{6}$ It was argued in [30] that since $\mathcal{N}_{2}-1$ is BRST trivial the amplitude would not be affected. For the three-loop, however, things are not so clear, as we will discuss in section 4.
} 
for the two-loop and

$$
\begin{aligned}
\left(d \gamma_{m n p} d\right) \doteq \quad & \left(d_{0} \gamma_{m n p} d_{0}\right) \oplus\left(d_{0}^{\prime} \gamma_{m n p} d_{0}^{\prime}\right) \oplus\left(d_{0}^{\prime \prime} \gamma_{m n p} d_{0}^{\prime \prime}\right) \\
& \oplus\left(d_{0} \gamma_{m n p} d_{0}^{\prime}\right) \oplus\left(d_{0} \gamma_{m n p} d_{0}^{\prime \prime}\right) \oplus\left(d_{0}^{\prime} \gamma_{m n p} d_{0}^{\prime \prime}\right)
\end{aligned}
$$

for the three-loop. At some point of the evaluation of a given amplitude, the zero-mode integrations need to be dealt with. Obviously the higher powers of the factor $\left(d \gamma_{m n p} d\right)$ and the larger numbers of the zero-modes are two of the causes that make a higher loop computation more complex than a tree- or one- loop amplitude.

One simplifying feature is that many of the $d$-zero structures that result from expanding factors of $\left(d \gamma_{m n p} d\right)$ actually vanish. This and some other aspects of the computation can be illustrated with the two-loop case. The zero mode part of the two-loop factor, $\left(d \gamma_{m n p} d\right)^{3}$, yields five different structures up to various interchanges of $d_{0}$ and $d_{0}^{\prime}$. The two-loop amplitude comes from the following structure

$$
\left(d_{0} \gamma_{m_{1} n_{1} p_{1}} d_{0}^{\prime}\right)\left(d_{0} \gamma_{m_{2} n_{2} p_{2}} d_{0}^{\prime}\right)\left(d_{0} \gamma_{m_{3} n_{3} p_{3}} d_{0}^{\prime}\right)
$$

which is shown to yield

$$
(r \lambda)\left(\lambda \gamma^{r} W_{(1)}\right)\left(\lambda \gamma^{q} W_{(3)}\right)\left(r W_{(4)}\right)\left(r \gamma^{q} \gamma^{r} W_{(2)}\right)
$$

in the next section. (Upon further evaluation, this yields the know two-loop result, (63).) Now we make the following important observations that are believed to remain true for any $d_{0}$-structure of any loop order. It is certainly true for the two- and all of the three- loop terms that we analyze in the next two sections. As mentioned above, the other four structures lead to vanishing results. The fact that they vanish is not so obvious in the beginning but becomes recognizable only after applying a serious of identities. The method presented in section 3.1 corresponds to keeping track of the overall numerical coefficient for a given $d_{0}$-structure. There is more than one way to evaluate a given $d_{0}$-structure. One may follow a more brute force type calculation where typically the expression under consideration breaks into many pieces. One can then simplify each contribution. The pattern that is observed is that they either yield zero or exactly the same expression as (24). What it means is that all of the five structures yield an expression that is proportional to (24) in a generic method of computation. It is only one of the structures given in (23) that yields a non-vanishing overall coefficient. We discuss this with an explicit example in section 4. (See footnote 11 for instance.) The structure of (23) is "tenacious": it is not so sensitive to the $d_{0}$-structures. Another important feature of the computations is that, typically, at the start of the simplification, the expression has more indices than those of (53) where there are two indices, r,q. All of the terms reduce to (53) or zero in the twoloop, regardless of how many indices they had initially. This is what happened in all examples analyzed in the three-loop case as well. They all reduce to (103) or zero. 
For the three-loop, there are additional complications that arise from the regulator issue. Unlike the two-loop case, no explicit gauge invariant regulator is known, at least not in a simple form. We will discuss the complications that are due to this lack of knowledge, but first will present the upshot of the analysis in the section 4. Based on the aforementioned tenaciousness of the amplitude structures, the following expression is proposed as an anticipated three-loop amplitude,

$$
(\lambda r)\left(\lambda \gamma^{t_{1}} \gamma^{t_{2}} r\right)[r W]\left[\lambda \gamma^{t_{3}} W\right]\left[r \gamma^{t_{1}} \gamma^{m^{\prime}} W\right]\left[r \gamma^{t_{2}} \gamma^{t_{3}} W\right]
$$

The expression is a three-loop analogue of (24). It should be relatively straightforward to put it in the form that corresponds to (63) and eventually further down to a full momentum space expression. For the remainder of this section, we outline the reasoning in section 4 that led to (25).

Setting apart the issue of gauge invariance, Berkovits and Aisaka have proposed a relatively simple three-loop regulator [34],

$$
\mathcal{N}_{3}=\quad \exp \left[-\sum_{I}^{3}\left(\bar{w}_{I}^{\alpha} w_{\alpha, I}+s_{I}^{\alpha} d_{\alpha, I}\right)\right] \exp \left(f^{\alpha} w_{\alpha}+g^{\alpha} d_{\alpha}+\bar{f}_{\alpha} \bar{w}^{\alpha}+\bar{g}_{\alpha} s^{\alpha}\right)
$$

elaborating on the work of [35]. The three-loop amplitude, $\mathcal{A}_{3-\text { loop }}$, given in (10) contains six insertions of the ghost field, $b$. The reason that the two-loop regulator, (18), is not sufficient for the three-loop computation can be seen through a "power counting" in $\frac{1}{\lambda \lambda}$. The six $b$-insertions produce several terms that would make the integration diverge. (This implies $0 \cdot \infty$ ambiguities because integrations over other fields give a vanishing result. Nevertheless it is useful to evaluate the three-loop with (18) inserted. The full justification will be given in section 4.) The (26) regulates the divergence by shifting $\lambda \bar{\lambda}$ by a constant. With use of (26), it is believed that the result of evaluation of the $\mathcal{A}_{3-\text { loop }}$ will be independent of the orders in which various integrations are performed. By performing the $d s$ - and $d d$ - integrations, it is easy to see that all the higher order $\frac{1}{\lambda \lambda}$-terms that have rendered the two-loop regulator invalid do not actually contribute ${ }^{7}$ : they simply vanish due to the $d_{0}$-mode saturation condition. This is curious since it will mean that the two-loop regulator, (18), will be equally good. In other words, the results of an evaluation of the $\mathcal{A}_{3-\text { loop }}$ using (18) and (26) are likely ${ }^{8}$ to be the same. The result of the evaluation with the two-loop regulator turns out to vanish as will be shown in section 4.1. This is an indication that the three-loop regulator (26) could lead to an entirely vanishing result. A few other indications will be discussed in the section 4 .

It is worth recalling what happened in the two-loop case. In the two-loop, even the structures that give vanishing results produce the two-loop amplitude, (24). It is just that the overall coefficients vanish. Therefore the question is whether there is a

\footnotetext{
${ }^{7}$ It would be more natural if the $\mathcal{A}_{3-\text { loop }}$ received some contributions from those higher order terms. We will discuss this issue further in the conclusion.

${ }^{8}$ There is a subtlety. It will be discussed in section 4 .
} 
tenacious structure - analogous to (24)- in the three-loop as well. The answer is yes. In section 4 , we take several $d_{0}$-structures out of $\left(d \gamma_{m n p} d\right)^{5}$ (also out of $\left.\left(d \gamma_{m n p} d\right)^{6}\right)$ and confirm that they all lead to zero or (25). To see (25) produced, one should follow a brute-force type analysis as in the two-loop analysis; namely the analysis in section 3.2. The status of the three-loop is different from that of the two-loop. First of all, there is a possibility that the three-loop regulator, (26), is incomplete as pointed out above. Secondly, in general there are far more terms to be evaluated. For one thing, there are more $d_{0}$-structure simply because there are more $d_{0}$-modes as can be seen from (22). The task of checking whether (26) indeed leads to a vanishing amplitude will require a huge amount of algebra, but so does $(26)$. Not only $\left(d \gamma_{m n p} d\right)^{5}$ generates many sub-cases to be analyzed. Fortunately, the tenaciousness that was observed in the two-loop analysis seems to persist in the three-loop as well. From accumulated experience, we believe that a certain pattern exists in the amplitude computation. (It may be viewed as a feature of the pure spinor formulation.) The pattern is that the amplitude structures such as (24) and (25) reside in many individual contributions even in their most broken down forms as sub-cases. It is not that several different $d_{0}$-structures must be combined to get (24) or (25). If true, it means that the role of a proper (or a gauge invariant) regulator is to make the overall coefficient non-vanishing. When expanded, the regulator, (26), generates many cases to be analyzed. In section 4 , we take a few random cases and carry out the analysis. Although we do not consider all the cases, those considered cover many other cases partially because of the powerful antisymmetry mentioned in (15).

\section{Direct two-loop computation}

In pure spinor literature, the two-loop amplitude was first obtained by a symmetry argument. Here we reproduce the result by a direct computation. The main purpose of the direct computation is to set the stage for a similar (but more complex) analysis of the three-loop in the next section. In the two-loop case, there are $32 d$-zero modes to saturate. The two-loop regulator, $\mathcal{N}_{2}$, provides 22 of them. The remaining $d$-zero modes should come from the $b$-ghosts and the vertex operators: the two-loop amplitude is

$$
\begin{aligned}
& \int[d \lambda][d \bar{\lambda}][d r]\left[d^{16} \theta\right] \prod_{I=1}^{2}\left[d w^{I}\right]\left[d \bar{w}^{I}\right]\left[d s^{I}\right]\left[d^{16} d^{I}\right] \\
& e^{-\bar{\lambda} \lambda-r \theta-\bar{w} w+s d} \\
& \frac{\left(\bar{\lambda} \gamma^{m_{1} n_{1} p_{1}} r\right)\left(d \gamma_{m_{1} n_{1} p_{1}} d\right)}{192(\bar{\lambda} \lambda)^{2}} \frac{\left(\bar{\lambda} \gamma^{m_{2} n_{2} p_{2}} r\right)\left(d \gamma_{m_{2} n_{2} p_{2}} d\right)}{192(\bar{\lambda} \lambda)^{2}} \frac{\left(\bar{\lambda} \gamma^{m_{3} n_{3} p_{3}} r\right)\left(d \gamma_{m_{3} n_{3} p_{3}} d\right)}{192(\bar{\lambda} \lambda)^{2}} \\
& {\left[d_{\alpha_{1}} W_{(1)}^{\alpha_{1}}\right]\left[d_{\alpha_{2}} W_{(2)}^{\alpha_{2}}\right]\left[d_{\alpha_{3}} W_{(3)}^{\alpha_{3}}\right]\left[d_{\alpha_{4}} W_{(4)}^{\alpha_{4}}\right]}
\end{aligned}
$$


where

$$
\left(d \gamma_{m_{i} n_{i} p_{i}} d\right) \sim \sum_{I, J}\left(d^{I} \gamma_{m_{i} n_{i} p_{i}} d^{J}\right)
$$

with $d^{I}(I=1,2)$ denoting the zero modes. (We often write $d^{I}$ instead of $d_{0}^{I}$ for the simplicity of notation.) The factor, $\left[d_{\alpha_{1}} W_{(1)}^{\alpha_{1}}\right]\left[d_{\alpha_{2}} W_{(2)}^{\alpha_{2}}\right]\left[d_{\alpha_{3}} W_{(3)}^{\alpha_{3}}\right]\left[d_{\alpha_{4}} W_{(4)}^{\alpha_{4}}\right]$, will give the sum of terms with different integrations on the moduli parameters. As for the kinematic factor, one can choose appropriate combinations in order to have overall $16 d^{I=1}$ 's and $16 d^{I=2}$ 's respectively. After the $\left[d s^{I}\right]$-integration, one gets

$$
\begin{gathered}
\frac{1}{(\bar{\lambda} \lambda)^{3}}\left[\left(\lambda \gamma^{r}\right)_{\alpha_{1}}\left(\lambda \gamma^{s}\right)_{\alpha_{2}}\left(\lambda \gamma^{q}\right)_{\alpha_{3}}\left(\gamma_{r s q}\right)_{\alpha_{4} \alpha_{5}} \epsilon^{\alpha_{1} \ldots \alpha_{5} \rho_{1} \ldots \rho_{11}} d_{\rho_{1}} \ldots d_{\rho_{11}}\right. \\
\left.\left(\lambda \gamma^{r^{\prime}}\right)_{\alpha_{1}^{\prime}}\left(\lambda \gamma^{s^{\prime}}\right)_{\alpha_{2}^{\prime}}\left(\lambda \gamma^{q^{\prime}}\right)_{\alpha_{3}^{\prime}}\left(\gamma_{r^{\prime} s^{\prime} q^{\prime}}\right)_{\alpha_{4}^{\prime} \alpha_{5}^{\prime}} \epsilon^{\alpha_{1}^{\prime} \ldots \alpha_{5}^{\prime} \rho_{1}^{\prime} \ldots \rho_{11}^{\prime}} d_{\rho_{1}^{\prime}}^{\prime} \ldots d_{\rho_{11}^{\prime}}^{\prime}\right] \\
\left(d \gamma_{m_{1} n_{1} p_{1}} d\right)\left(d \gamma_{m_{2} n_{2} p_{2}} d\right)\left(d \gamma_{m_{3} n_{3} p_{3}} d\right)\left(\bar{\lambda} \gamma^{m_{1} n_{1} p_{1}} r\right)\left(\bar{\lambda} \gamma^{m_{2} n_{2} p_{2}} r\right)\left(\bar{\lambda} \gamma^{m_{3} n_{3} p_{3}} r\right) \\
{\left[d_{\alpha_{1}} W_{(1)}^{\alpha_{1}}\right]\left[d_{\alpha_{2}} W_{(2)}^{\alpha_{2}}\right]\left[d_{\alpha_{3}} W_{(3)}^{\alpha_{3}}\right]\left[d_{\alpha_{4}} W_{(4)}^{\alpha_{4}}\right]}
\end{gathered}
$$

where in the abuse of the notation, we have defined

$$
\begin{aligned}
d^{I=1} & \equiv d \\
d^{I=2} & \equiv d^{\prime}
\end{aligned}
$$

To saturate the $\left[d d^{I}\right]$-integral, $16 d$ 's and $16 d^{\prime \prime}$ 's must be present. Expansion of

$$
\left(d \gamma_{m_{1} n_{1} p_{1}} d\right)\left(d \gamma_{m_{2} n_{2} p_{2}} d\right)\left(d \gamma_{m_{3} n_{3} p_{3}} d\right)
$$

in terms of the zero modes yields several terms. Each term comes with an overall coefficient function of moduli parameters. In all of the subsequent discussions, we omit those factors. There are five different types of terms ${ }^{9}$ : the following types of terms

$$
\begin{aligned}
& \left(d \gamma_{m_{1} n_{1} p_{1}} d\right)\left(d \gamma_{m_{2} n_{2} p_{2}} d\right)\left(d^{\prime} \gamma_{m_{3} n_{3} p_{3}} d^{\prime}\right) \\
& \left(d \gamma_{m_{1} n_{1} p_{1}} d\right)\left(d \gamma_{m_{2} n_{2} p_{2}} d\right)\left(d \gamma_{m_{3} n_{3} p_{3}} d^{\prime}\right) \\
& \left(d \gamma_{m_{1} n_{1} p_{1}} d\right)\left(d^{\prime} \gamma_{m_{2} n_{2} p_{2}} d^{\prime}\right)\left(d \gamma_{m_{3} n_{3} p_{3}} d^{\prime}\right) \\
& \left(d \gamma_{m_{1} n_{1} p_{1}} d^{\prime}\right)\left(d \gamma_{m_{2} n_{2} p_{2}} d^{\prime}\right)\left(d \gamma_{m_{3} n_{3} p_{3}} d\right)
\end{aligned}
$$

lead to vanishing results as we show in the section below. The fifth type of term

$$
\left(d \gamma_{m_{1} n_{1} p_{1}} d^{\prime}\right)\left(d \gamma_{m_{2} n_{2} p_{2}} d^{\prime}\right)\left(d \gamma_{m_{3} n_{3} p_{3}} d^{\prime}\right)
$$

produces the expected two-loop amplitude. We will work out that part of the computation in the section that follows the next one.

\footnotetext{
${ }^{9}$ There also terms that can be obtained by interchanging the roles of $d$ and $d^{\prime}$. At the end, they will only change the overall numerical coefficient of the computation of each type.
} 


\section{1 terms that vanish: analysis of eq.(32)}

We illustrate the vanishing of the terms in (32) with two examples, the first term and the third term. While doing so, we derive two identities that will be heavily used in the three-loop analysis as well. The computation of the term that involves

$$
\left(d \Gamma_{m_{1} n_{1} p_{1}} d\right)\left(d \Gamma_{m_{2} n_{2} p_{2}} d\right)\left(d^{\prime} \Gamma_{m_{3} n_{3} p_{3}} d^{\prime}\right)
$$

goes as follows. Integrating over the measure $[d s]$, one gets

$$
\begin{gathered}
\frac{1}{(\bar{\lambda} \lambda)^{3}}\left[\left(\lambda \gamma^{r}\right)_{\alpha_{1}}\left(\lambda \gamma^{s}\right)_{\alpha_{2}}\left(\lambda \gamma^{q}\right)_{\alpha_{3}}\left(\gamma_{r s q}\right)_{\alpha_{4} \alpha_{5}} \epsilon^{\alpha_{1} \ldots \alpha_{5} \rho_{1} \ldots \rho_{11}} d_{\rho_{1} \ldots} \ldots d_{\rho_{11}}\right. \\
\left.\left(\lambda \gamma^{r^{\prime}}\right)_{\alpha_{1}^{\prime}}\left(\lambda \gamma^{s^{\prime}}\right)_{\alpha_{2}^{\prime}}\left(\lambda \gamma^{q^{\prime}}\right)_{\alpha_{3}^{\prime}}\left(\gamma_{r^{\prime} s^{\prime} q^{\prime}}\right)_{\alpha_{4}^{\prime} \alpha_{5}^{\prime}} \epsilon^{\alpha_{1}^{\prime} \ldots \alpha_{5}^{\prime} \rho_{1}^{\prime} \ldots \rho_{11}^{\prime}} d_{\rho_{1}^{\prime}}^{\prime} \ldots d_{\rho_{11}^{\prime}}^{\prime}\right] \\
\left(d \gamma_{m_{1} n_{1} p_{1}} d\right)\left(d \gamma_{m_{2} n_{2} p_{2}} d\right)\left(d^{\prime} \gamma_{m_{3} n_{3} p_{3}} d^{\prime}\right)\left(\bar{\lambda} \gamma^{m_{1} n_{1} p_{1}} r\right)\left(\bar{\lambda} \gamma^{m_{2} n_{2} p_{2}} r\right)\left(\bar{\lambda} \gamma^{m_{3} n_{3} p_{3}} r\right) \\
{\left[d_{\alpha_{1}} W_{(1)}^{\alpha_{1}}\right]\left[d_{\alpha_{2}} W_{(2)}^{\alpha_{2}}\right]\left[d_{\alpha_{3}} W_{(3)}^{\alpha_{3}}\right]\left[d_{\alpha_{4}} W_{(4)}^{\alpha_{4}}\right]}
\end{gathered}
$$

Performing the $[d d]$-integration and utilizing the freedom mentioned in (15), the relevant part of the above equation becomes

$$
\begin{aligned}
& \left(\lambda \gamma^{r} \gamma_{m_{1} n_{1} p_{1}} \gamma^{s} \lambda\right)\left(\lambda \gamma^{q} W^{(1)}\right) \operatorname{Tr}\left(\gamma_{m_{2} n_{2} p_{2}} \gamma_{r s q}\right) \\
& \left(\lambda \gamma^{r^{\prime}} \gamma_{m_{3} n_{3} p_{3}} \gamma^{s^{\prime}} \lambda\right)\left(W^{(2)} \gamma_{r^{\prime} s^{\prime} q^{\prime}} W^{(3)}\right)\left(\lambda \gamma^{q^{\prime}} W^{(4)}\right) \\
& \left(\bar{\lambda} \gamma^{m_{1} n_{1} p_{1}} r\right)\left(\bar{\lambda} \gamma^{m_{2} n_{2} p_{2}} r\right)\left(\bar{\lambda} \gamma^{m_{3} n_{3} p_{3}} r\right)
\end{aligned}
$$

In the 32-component notation, it takes the form of

$$
\begin{aligned}
& \left(\lambda_{u} \Gamma^{r} \Gamma_{m_{1} n_{1} p_{1}} \Gamma^{s} \lambda_{d}\right)\left(\lambda_{u} \Gamma^{q} W_{d}^{(1)}\right) \\
& \left(\lambda_{u} \Gamma^{r^{\prime}} \Gamma_{m_{3} n_{3} p_{3}} \Gamma^{s^{\prime}} \lambda_{d}\right)\left(W_{u}^{(2)} \Gamma_{r^{\prime} s^{\prime} q^{\prime}} W_{d}^{(3)}\right)\left(\lambda_{u} \Gamma^{q^{\prime}} W_{d}^{(4)}\right) \\
& \left(\bar{\lambda}_{d} \Gamma^{m_{1} n_{1} p_{1}} r_{u}\right)\left(\bar{\lambda}_{d} \Gamma^{r s q} r_{u}\right)\left(\bar{\lambda}_{d} \Gamma^{m_{3} n_{3} p_{3}} r_{u}\right)
\end{aligned}
$$

Using one of the 16-component Fierz identities, (B.4),

$$
\gamma_{\alpha \beta}^{m} \gamma_{\delta \sigma}^{m}=-\frac{1}{2} \gamma_{\alpha \delta}^{m} \gamma_{\beta \sigma}^{m}+\frac{1}{24} \gamma_{\alpha \delta}^{m n p} \gamma_{\sigma \beta}^{m n p}
$$

one can easily obtain the 16-component version of

$$
\left(\bar{\lambda}_{d} \Gamma^{m n p} r_{u}\right)\left(\lambda_{u} \Gamma^{r} \Gamma_{m n p} \Gamma^{s} \lambda_{d}\right)=-48\left(\bar{\lambda}_{d} \Gamma^{s} \Gamma^{r} \lambda_{d}\right)\left(\lambda_{u} r_{u}\right)+48(\bar{\lambda} \lambda)\left(\lambda_{u} \Gamma^{r} \Gamma^{s} r_{u}\right)
$$

Both of these terms yield vanishing expressions. The first term with another factor in (37), $\left(\bar{\lambda}_{d} \Gamma^{r s q} r_{u}\right)$, leads to a vanishing expression,

$$
\left(\bar{\lambda}_{d} \Gamma^{s} \Gamma^{r} \lambda_{d}\right)\left(\bar{\lambda}_{d} \Gamma^{r s q} r_{u}\right)=0
$$


due to one of the pure spinor constraints. The second term of (39) gives, up to an overall numerical factor,

$$
\begin{aligned}
& (\bar{\lambda} \lambda)^{2}\left(\lambda_{u} \Gamma^{r} \Gamma^{s} r_{u}\right)\left(\lambda_{u} \Gamma^{r^{\prime}} \Gamma^{s^{\prime}} r_{u}\right)\left(\lambda_{u} \Gamma^{q} W_{d}^{(1)}\right) \\
& \left(W_{u}^{(2)} \Gamma_{r^{\prime} s^{\prime} q^{\prime}} W_{d}^{(3)}\right)\left(\lambda_{u} \Gamma^{q^{\prime}} W_{d}^{(4)}\right)\left(\bar{\lambda}_{d} \Gamma^{r s q} r_{u}\right)
\end{aligned}
$$

It contains the factors $\left(\bar{\lambda}_{d} \Gamma^{r s q} r_{u}\right)\left(\lambda_{u} \Gamma^{r} \Gamma^{s} r_{u}\right)$. By Fierzing, it is not difficult to see that it vanishes:

$$
\left(\bar{\lambda}_{d} \Gamma^{r s q} r_{u}\right)\left(\lambda_{u} \Gamma^{r} \Gamma^{s} r_{u}\right)=0
$$

There are a few different ways to prove it. The proof that we adopt shows the interplay between the 16- and 32- component notations. The anti-symmetry of the first factor allows one to write the second factor as $\left(\lambda_{u} \Gamma^{r s} r_{u}\right)$. Because of the constraint, $\left(\bar{\lambda}_{d} \Gamma^{m} r_{u}\right)=0$, the left hand side can be rewritten as

$$
\begin{aligned}
& \sim\left(\bar{\lambda}_{d} \Gamma^{q} \Gamma^{r s} r_{u}\right)\left(\lambda_{u} \Gamma^{r s} r_{u}\right) \\
& \sim\left(\bar{\lambda} \gamma^{q} \gamma^{r s} r\right)\left(\lambda \gamma^{r s} r\right)
\end{aligned}
$$

The 16-dimensional gamma matrix identity [35],

$$
\left(\gamma^{m n}\right)_{\alpha}^{\gamma}\left(\gamma_{m n}\right)_{\beta}{ }^{\sigma}=-8 \delta_{\alpha}{ }^{\sigma} \delta_{\beta}^{\gamma}-2 \delta_{\alpha}^{\gamma} \delta_{\beta}{ }^{\sigma}+4\left(\gamma^{p}\right)_{\alpha \beta}\left(\gamma_{p}\right)^{\gamma \sigma}
$$

can now be used in the second equation of (43) to complete the proof. To see the third term of $(32)$

$$
\left(d \gamma_{m_{1} n_{1} p_{1}} d\right)\left(d^{\prime} \gamma_{m_{2} n_{2} p_{2}} d^{\prime}\right)\left(d \gamma_{m_{3} n_{3} p_{3}} d^{\prime}\right)
$$

vanish, let us use the identity (B.4) (quoted in (38)) in

$$
\begin{aligned}
& \left(\gamma_{m_{1} n_{1} p_{1}}\right)^{\kappa_{1} \kappa_{2}}\left(\gamma_{m_{2} n_{2} p_{2}}\right)^{\kappa_{1}^{\prime} \kappa_{2}^{\prime}}\left(\gamma_{m_{3} n_{3} p_{3}}\right)^{\kappa_{3} \kappa_{3}^{\prime}} \\
& \left(\bar{\lambda} \gamma^{m_{1} n_{1} p_{1}} r\right)\left(\bar{\lambda} \gamma^{m_{2} n_{2} p_{2}} r\right)\left(\bar{\lambda} \gamma^{m_{3} n_{3} p_{3}} r\right)
\end{aligned}
$$

where the $d$-factors have been suppressed. Taking the pure spinor constraint, $\left(\bar{\lambda} \gamma^{p} r\right)=$ 0 , the above equation simplifies to

$$
\left(r \gamma^{u_{1}}\right)^{\kappa_{1}}\left(\bar{\lambda} \gamma_{u_{1}}\right)^{\kappa_{2}}\left(r \gamma^{u_{2}}\right)^{\kappa_{1}^{\prime}}\left(\bar{\lambda} \gamma_{u_{2}}\right)^{\kappa_{2}^{\prime}}\left(r \gamma^{u_{3}}\right)^{\kappa_{3}}\left(\bar{\lambda} \gamma_{u_{3}}\right)^{\kappa_{3}^{\prime}}
$$

After performing $[d s]$ - and $[d d]$ - integrations, one can choose (again thanks to the freedom stated in (15)) the contractions with $\lambda$-factors to get

$$
\begin{aligned}
& \left(r \gamma^{r s q} \bar{\lambda}\right)\left(r \gamma^{r^{\prime} s^{\prime} q^{\prime}} \bar{\lambda}\right)\left(r \gamma^{u_{3}} \gamma^{r} \lambda\right)\left(\bar{\lambda} \gamma_{u_{3}} \gamma^{r^{\prime}} \lambda\right) \\
& \sim(\bar{\lambda} \lambda)\left(r \gamma^{r s q} \bar{\lambda}\right)\left(r \gamma^{r^{\prime} s^{\prime} q^{\prime}} \bar{\lambda}\right)\left(r \gamma^{r^{\prime}} \gamma^{r} \lambda\right)
\end{aligned}
$$

Since the factor $\left(d \gamma_{m_{3} n_{3} p_{3}} d^{\prime}\right)$ in (45) can be written as $\left(d^{\prime} \gamma_{m_{3} n_{3} p_{3}} d\right)$, the factor, $\left(r \gamma^{r^{\prime}} \gamma^{r} \lambda\right)$, can be replaced by $\eta^{r^{\prime} r}(r \lambda)$. Once used in the equation above, the resulting expression vanishes due to the constraint, $\left(\ldots \gamma^{r} \bar{\lambda}\right)\left(\ldots \gamma_{r} \bar{\lambda}\right)=0$. 


\section{2 the two-loop amplitude from eq.(33)}

The two-loop amplitude is produced by the term in (33)

$$
\left(d \gamma_{m_{1} n_{1} p_{1}} d^{\prime}\right)\left(d \gamma_{m_{2} n_{2} p_{2}} d^{\prime}\right)\left(d \gamma_{m_{3} n_{3} p_{3}} d^{\prime}\right)
$$

After carrying out the $d$-integration, one can choose the index contractions appropriately so as to maximize the usage of (39). There are several different type of terms depending on the frequency of appearance of either the first or the second term of (39). The first term cannot appear more than once since otherwise the resulting expression would contain

$$
(\lambda r)(\lambda r)=0
$$

due to the (anti-)commutativity of $\lambda$ and $r$. Therefore there are two types of terms

$$
\begin{aligned}
& (\bar{\lambda} \lambda)^{2}(\lambda r)\left(\bar{\lambda} \gamma^{r^{\prime}} \gamma^{r} \lambda\right)\left(\lambda \gamma^{s} \gamma^{s^{\prime}} r\right)\left(\lambda \gamma^{q} \gamma^{q^{\prime}} r\right)\left[W^{(1)} \gamma_{r} \gamma_{s} \gamma_{q} W^{(2)}\right]\left[W^{(3)} \gamma_{r^{\prime}} \gamma_{s^{\prime}} \gamma_{q^{\prime}} W^{(4)}\right] \\
& (\bar{\lambda} \lambda)^{3}\left(\lambda \gamma^{r} \gamma^{r^{\prime}} r\right)\left(\lambda \gamma^{s} \gamma^{s^{\prime}} r\right)\left(\lambda \gamma^{q} \gamma^{q^{\prime}} r\right)\left[W^{(1)} \gamma_{r} \gamma_{s} \gamma_{q} W^{(2)}\right]\left[W^{(3)} \gamma_{r^{\prime}} \gamma_{s^{\prime}} \gamma_{q^{\prime}} W^{(4)}\right]
\end{aligned}
$$

Up to an overall numerical factor they give the same contributions: We illustrate the computation with the second term. To apply (5), we rewrite the second term,

$$
\begin{aligned}
& (\bar{\lambda} \lambda)^{3} \lambda^{\alpha_{1}}\left(\gamma^{r^{\prime}}\right)^{\alpha_{2} \alpha_{3}} r_{\alpha_{3}} \lambda^{\beta_{1}}\left(\gamma^{s}\right)_{\beta_{1} \beta_{2}} r_{\beta_{3}} \lambda^{\gamma_{1}}\left(\gamma^{q^{\prime}}\right)^{\gamma_{2} \gamma_{3}} r_{\gamma_{3}} \\
& W_{(1)}^{\rho_{1}}\left(\gamma_{s}\right)^{\rho_{2} \rho_{3}} W_{(2)}^{\rho_{4}} W_{(3)}{ }^{\sigma_{1}}\left(\gamma_{r^{\prime}}\right)_{\sigma_{1} \sigma_{2}}\left(\gamma_{q^{\prime}}\right)_{\sigma_{3} \sigma_{4}} W_{(4)}^{\sigma_{4}} \\
& \left(\gamma^{r}\right)_{\alpha_{1} \alpha_{2}}\left(\gamma_{r}\right)_{\rho_{1} \rho_{2}}\left(\gamma^{q}\right)_{\gamma_{1} \gamma_{2}}\left(\gamma_{q}\right)_{\rho_{3} \rho_{4}}\left(\gamma^{s^{\prime}}\right)^{\beta_{2} \beta_{3}}\left(\gamma_{s^{\prime}}\right)^{\sigma_{2} \sigma_{3}}
\end{aligned}
$$

Some of the terms that result by using (5) in the third line vanish. The surviving terms can be put into

$$
(r \lambda)\left(\lambda \gamma^{r} W_{(1)}\right)\left(\lambda \gamma^{q} W_{(3)}\right)\left(r W_{(4)}\right)\left(r \gamma^{q} \gamma^{r} W_{(2)}\right)
$$

Up to an irrelevant overall numerical factor, it can be put into

$$
\begin{aligned}
& \left(\lambda \gamma^{r}\right)_{\beta_{1}}\left(\lambda \gamma^{q}\right)_{\beta_{3}}\left(\gamma^{q} \gamma^{r}\right)_{\beta_{2}}^{\alpha_{2}} \lambda^{\alpha_{1}} \\
& D_{\alpha_{2}} D_{\alpha_{1}} D_{\beta_{4}}\left[W_{(1)}^{\beta_{1}} W_{(2)}^{\beta_{2}} W_{(3)}^{\beta_{3}} W_{(4)}^{\beta_{4}}\right]
\end{aligned}
$$

There are three types of terms depending how the covariant derivatives are distributed: the first type of terms is such that all $D^{\prime}$ 's act on different $W^{\prime} s$. (Recall that due to the field equation, one has $D_{\beta_{4}} W_{(4)}^{\beta^{4}}=0$.) These are the terms that produce the non-zero result as we will discuss shortly. The other two types of terms are the ones with all three $D$ 's acting on the same $W$ and the ones with two $D$ 's acting on the same $W$ and the third $D$ acting on another $W$. They all vanish due to either Bianchi identity or the presence of a vanishing factor $\left(\lambda \gamma^{m n p} \lambda\right)$. 
Let us consider each type of the term. The first is the type of the terms where all three $D$ 's act on the same $W$. The terms that contain $D_{\alpha_{2}} D_{\alpha_{1}} D_{\beta_{4}} W_{(1)}^{\beta_{1}}$ lead to

$$
\doteq k_{l_{1}}^{(1)} \mathcal{F}_{v_{1} v_{2}}^{(1)}\left(\lambda \gamma^{v_{1}} W_{(2)}\right)\left(\lambda \gamma^{v_{2}} W_{(3)}\right)\left(\lambda \gamma^{l_{1}} W_{(4)}\right)
$$

The indices, $\left(l_{1}, v_{1}, v_{2}\right)$, will be anti-symmetrized once the permutations in $\left({ }_{(1)},(2),(3),(4)\right)$ are taken into account, so the equation above vanishes due to Bianchi identity. The term containing $D_{\alpha_{2}} D_{\alpha_{1}} D_{\beta_{4}} W_{(2)}^{\beta_{2}}$ directly leads to $k_{\left[l_{1}\right.}^{(2)} \mathcal{F}_{\left.v_{1} v_{2}\right]}^{(2)}$, therefore vanishes. The terms containing $D_{\alpha_{2}} D_{\alpha_{1}} D_{\beta_{4}} W_{(3)}^{\beta_{3}}$ are given by $(1 \leftrightarrow 3)$ of $(55)$. The terms with two $D$ 's acting on the same $W$ and the third $D$ acting on another $W$ produces a similar result. For example the term with $\left(D_{\alpha_{1}} D_{\beta_{4}} W_{(1)}^{\beta_{1}}\right)\left(D_{\alpha_{2}} W_{(2)}^{\beta_{2}}\right) W_{(3)}^{\beta_{3}} W_{(4)}^{\beta_{4}}$ yields

$$
\doteq\left(\lambda \gamma^{m} W^{(1)}\right)\left(\lambda \gamma^{n} W^{(3)}\right)\left(\lambda \gamma^{p} W^{(4)}\right) k_{p}^{(1)} \mathcal{F}_{m n}^{(2)}
$$

Consider another example, $\left(D_{\alpha_{1}} D_{\beta_{4}} W_{(1)}^{\beta_{1}}\right) W_{(2)}^{\beta_{2}}\left(D_{\alpha_{2}} W_{(3)}^{\beta_{3}}\right) W_{(4)}^{\beta_{4}}$. It yields

$$
\doteq\left(\lambda \gamma^{m} W^{(4)}\right)\left(\lambda \gamma^{u} W^{(2)}\right)\left(\lambda \gamma^{v} \partial_{m} W^{(1)}\right) \mathcal{F}_{u v}^{(3)}
$$

There are altogether 27 terms of this type. After some algebra one can show

$$
\begin{aligned}
& -D_{\alpha_{1}} D_{\beta_{4}} W_{(1)}^{\beta_{1}} D_{\alpha_{2}} W_{(2)}^{\beta_{2}} W_{(3)}^{\beta_{3}} W_{(4)}^{\beta_{4}}+D_{\alpha_{1}} D_{\beta_{4}} W_{(1)}^{\beta_{1}} W_{(2)}^{\beta_{2}} D_{\alpha_{2}} W_{(3)}^{\beta_{3}} W_{(4)}^{\beta_{4}} \\
& -D_{\alpha_{1}} D_{\beta_{4}} W_{(1)}^{\beta_{1}} W_{(2)}^{\beta_{2}} W_{(3)}^{\beta_{3}} D_{\alpha_{2}} W_{(4)}^{\beta_{4}} \\
& +D_{\alpha_{2}} D_{\beta_{4}} W_{(1)}^{\beta_{1}} D_{\alpha_{1}} W_{(2)}^{\beta_{2}} W_{(3)}^{\beta_{3}} W_{(4)}^{\beta_{4}}+D_{\beta_{4}} W_{(1)}^{\beta_{1}} D_{\alpha_{2}} D_{\alpha_{1}} W_{(2)}^{\beta_{2}} W_{(3)}^{\beta_{3}} W_{(4)}^{\beta_{4}} \\
& -D_{\alpha_{2}} D_{\beta_{4}} W_{(1)}^{\beta_{1}} W_{(2)}^{\beta_{2}} D_{\alpha_{1}} W_{(3)}^{\beta_{3}} W_{(4)}^{\beta_{4}}+D_{\beta_{4}} W_{(1)}^{\beta_{1}} W_{(2)}^{\beta_{2}} D_{\alpha_{2}} D_{\alpha_{1}} W_{(3)}^{\beta_{3}} W_{(4)}^{\beta_{4}} \\
& +D_{\alpha_{2}} D_{\beta_{4}} W_{(1)}^{\beta_{1}} W_{(2)}^{\beta_{2}} W_{(3)}^{\beta_{3}} D_{\alpha_{1}} W_{(4)}^{\beta_{4}}+D_{\beta_{4}} W_{(1)}^{\beta_{1}} W_{(2)}^{\beta_{2}} W_{(3)}^{\beta_{3}} D_{\alpha_{2}} D_{\alpha_{1}} W_{(4)}^{\beta_{4}} \\
& -D_{\alpha_{2}} D_{\alpha_{1}} W_{(1)}^{\beta_{1}} D_{\beta_{4}} W_{(2)}^{\beta_{2}} W_{(3)}^{\beta_{3}} W_{(4)}^{\beta_{4}}-D_{\alpha_{1}} W_{(1)}^{\beta_{1}} D_{\alpha_{2}} D_{\beta_{4}} W_{(2)}^{\beta_{2}} W_{(3)}^{\beta_{3}} W_{(4)}^{\beta_{4}} \\
& +D_{\alpha_{2}} W_{(1)}^{\beta_{1}} D_{\alpha_{1}} D_{\beta_{4}} W_{(2)}^{\beta_{2}} W_{(3)}^{\beta_{3}} W_{(4)}^{\beta_{4}}+W_{(1)}^{\beta_{1}} D_{\alpha_{1}} D_{\beta_{4}} D_{\beta_{4}} W_{(2)}^{\beta_{2}} D_{\alpha_{2}} W_{(3)}^{\beta_{3}} W_{(4)}^{\beta_{4}} \\
& -W_{(1)}^{\beta_{1}} D_{\alpha_{1}} D_{\beta_{4}} W_{(2)}^{\beta_{2}} W_{(3)}^{\beta_{3}} D_{\alpha_{2}} W_{(4)}^{\beta_{4}} \\
& -W_{(1)}^{\beta_{1}} D_{\alpha_{2}} D_{\beta_{4}} W_{(2)}^{\beta_{2}} D_{\alpha_{1}} W_{(3)}^{\beta_{3}} W_{(4)}^{\beta_{4}}-W_{(1)}^{\beta_{1}} D_{\beta_{4}} W_{(2)}^{\beta_{2}} D_{\alpha_{2}} D_{\alpha_{1}} W_{(3)}^{\beta_{3}} W_{(4)}^{\beta_{4}} \\
& +W_{(1)}^{\beta_{1}} D_{\alpha_{2}} D_{\beta_{4}} W_{(2)}^{\beta_{2}} W_{(3)}^{\beta_{3}} D_{\alpha_{1}} W_{(4)}^{\beta_{4}}-W_{(1)}^{\beta_{1}} D_{\beta_{4}} W_{(2)}^{\beta_{2}} W_{(3)}^{\beta_{3}} D_{\alpha_{2}} D_{\alpha_{1}} W_{(4)}^{\beta_{4}} \\
& +D_{\alpha_{2}} D_{\alpha_{1}} W_{(1)}^{\beta_{1}} W_{(2)}^{\beta_{2}} D_{\beta_{4}} W_{(3)}^{\beta_{3}} W_{(4)}^{\beta_{4}}-D_{\alpha_{1}} W_{(1)}^{\beta_{1}} W_{(2)}^{\beta_{2}} D_{\alpha_{2}} D_{\beta_{4}} W_{(3)}^{\beta_{3}} W_{(4)}^{\beta_{4}} \\
& +W_{(1)}^{\beta_{1}} D_{\alpha_{2}} D_{\alpha_{1}} W_{(2)}^{\beta_{2}} D_{\beta_{4}} W_{(3)}^{\beta_{3}} W_{(4)}^{\beta_{4}}+W_{(1)}^{\beta_{1}} D_{\alpha_{1}} W_{(2)}^{\beta_{2}} D_{\alpha_{2}} D_{\beta_{4}} W_{(3)}^{\beta_{3}} W_{(4)}^{\beta_{4}} \\
& +D_{\alpha_{2}} W_{(1)}^{\beta_{1}} W_{(2)}^{\beta_{2}} D_{\alpha_{1}} D_{\beta_{4}} W_{(3)}^{\beta_{3}} W_{(4)}^{\beta_{4}}-W_{(1)}^{\beta_{1}} D_{\alpha_{2}} W_{(2)}^{\beta_{2}} D_{\alpha_{1}} D_{\beta_{4}} W_{(3)}^{\beta_{3}} W_{(4)}^{\beta_{4}} \\
& -W_{(1)}^{\beta_{1}} W_{(2)}^{\beta_{2}} D_{\alpha_{1}} D_{\beta_{4}} W_{(3)}^{\beta_{3}} D_{\alpha_{2}} W_{(4)}^{\beta_{4}} \\
& +W_{(1)}^{\beta_{1}} W_{(2)}^{\beta_{2}} D_{\alpha_{2}} D_{\beta_{4}} W_{(3)}^{\beta_{3}} D_{\alpha_{1}} W_{(4)}^{\beta_{4}}+W_{(1)}^{\beta_{1}} W_{(2)}^{\beta_{2}} D_{\beta_{4}} W_{(3)}^{\beta_{3}} D_{\alpha_{2}} D_{\alpha_{1}} W_{(4)}^{\beta_{4}} \\
& =\left(\lambda \gamma^{m} W_{(1)}\right)\left(\lambda \gamma^{n} W_{(3)}\right)\left(\lambda \gamma^{p} W_{(4)}\right)\left[-6 k_{p}^{(2)} \mathcal{F}_{m n}^{(2)}-4 k_{n}^{(4)} \mathcal{F}_{m p}^{(2)}+4 k_{m}^{(4)} \mathcal{F}_{n p}^{(2)}\right] \\
& +\left(\lambda \gamma^{m} W_{(1)}\right)\left(\lambda \gamma^{n} W_{(2)}\right)\left(\lambda \gamma^{p} W_{(4)}\right)\left[-6 k_{p}^{(3)} \mathcal{F}_{m n}^{(3)}+4 k_{m}^{(4)} \mathcal{F}_{n p}^{(3)}-4 k_{n}^{(4)} \mathcal{F}_{m p}^{(3)}\right] \\
& +\left(\lambda \gamma^{m} W_{(1)}\right)\left(\lambda \gamma^{n} W_{(2)}\right)\left(\lambda \gamma^{p} W_{(3)}\right)\left[-2 k_{p}^{(4)} \mathcal{F}_{m n}^{(4)}+2 k_{n}^{(4)} \mathcal{F}_{m p}^{(4)}-2 k_{m}^{(4)} \mathcal{F}_{n p}^{(4)}\right] \\
& +\left(\lambda \gamma^{m} W_{(2)}\right)\left(\lambda \gamma^{n} W_{(3)}\right)\left(\lambda \gamma^{p} W_{(4)}\right)\left[-6 k_{p}^{(1)} \mathcal{F}_{m n}^{(1)}-4 k_{n}^{(4)} \mathcal{F}_{m p}^{(1)}+4 k_{m}^{(4)} \mathcal{F}_{n p}^{(1)}\right]
\end{aligned}
$$


The third term vanishes due to Bianchi identity, $k_{[m} \mathcal{F}_{n p]}=0$. The terms with the factors, $k_{p}^{(1)} \mathcal{F}_{m n}^{(1)}, k_{p}^{(2)} \mathcal{F}_{m n}^{(2)}, k_{p}^{(3)} \mathcal{F}_{m n}^{(3)}$, vanish after taking the permutations over $(1,2,3,4)$ into account. The remaining terms are

$$
\begin{aligned}
& \left(\lambda \gamma^{m} W_{(1)}\right)\left(\lambda \gamma^{n} W_{(3)}\right)\left(\lambda \gamma^{p} W_{(4)}\right)\left[-4 k_{n}^{(4)} \mathcal{F}_{m p}^{(2)}+4 k_{m}^{(4)} \mathcal{F}_{n p}^{(2)}\right] \\
& +\left(\lambda \gamma^{m} W_{(1)}\right)\left(\lambda \gamma^{n} W_{(2)}\right)\left(\lambda \gamma^{p} W_{(4)}\right)\left[4 k_{m}^{(4)} \mathcal{F}_{n p}^{(3)}-4 k_{n}^{(4)} \mathcal{F}_{m p}^{(3)}\right] \\
& +\left(\lambda \gamma^{m} W_{(2)}\right)\left(\lambda \gamma^{n} W_{(3)}\right)\left(\lambda \gamma^{p} W_{(4)}\right)\left[-4 k_{n}^{(4)} \mathcal{F}_{m p}^{(1)}+4 k_{m}^{(4)} \mathcal{F}_{n p}^{(1)}\right]
\end{aligned}
$$

The second term is obtained from the first term by $(2 \leftrightarrow 3)$ and the third term is obtained from the first term by $(1 \leftrightarrow 2)$. Therefore we may consider the first term only. The first term is symmetric under $(1 \leftrightarrow 3)$ : it is sufficient to consider

$$
\left(\lambda \gamma^{m} W_{(1)}\right)\left(\lambda \gamma^{n} W_{(3)}\right)\left(\lambda \gamma^{p} W_{(4)}\right) k_{n}^{(4)} \mathcal{F}_{m p}^{(2)}
$$

with its permutations in $(1,2,3,4)$, altogether 24 terms. We put them into four groups: the first group includes the terms with $\mathcal{F}_{m p}^{(1)}$, the second group $\mathcal{F}_{m p}^{(2)}$, the third group $\mathcal{F}_{m p}^{(3)}$ and the fourth group $\mathcal{F}_{m p}^{(2)}$. Each group has a vanishing result. We illustrate this with the second group. The six terms in the second group are

$$
\begin{aligned}
& \left(\lambda \gamma^{m} W_{(1)}\right)\left(\lambda \gamma^{n} W_{(3)}\right)\left(\lambda \gamma^{p} W_{(4)}\right) \mathcal{F}_{m p}^{(2)} k_{n}^{(4)}+\left(\lambda \gamma^{m} W_{(1)}\right)\left(\lambda \gamma^{n} W_{(4)}\right)\left(\lambda \gamma^{p} W_{(3)}\right) \mathcal{F}_{m p}^{(2)} k_{n}^{(3)} \\
& +\left(\lambda \gamma^{m} W_{(3)}\right)\left(\lambda \gamma^{n} W_{(4)}\right)\left(\lambda \gamma^{p} W_{(1)}\right) \mathcal{F}_{m p}^{(2)} k_{n}^{(1)}+\left(\lambda \gamma^{m} W_{(3)}\right)\left(\lambda \gamma^{n} W_{(1)}\right)\left(\lambda \gamma^{p} W_{(4)}\right) \mathcal{F}_{m p}^{(2)} k_{n}^{(4)} \\
& +\left(\lambda \gamma^{m} W_{(4)}\right)\left(\lambda \gamma^{n} W_{(1)}\right)\left(\lambda \gamma^{p} W_{(3)}\right) \mathcal{F}_{m p}^{(2)} k_{n}^{(3)}+\left(\lambda \gamma^{m} W_{(4)}\right)\left(\lambda \gamma^{n} W_{(3)}\right)\left(\lambda \gamma^{p} W_{(1)}\right) \mathcal{F}_{m p}^{(2)} k_{n}^{(1)} \\
\doteq \quad & \left(\lambda \gamma^{m} W_{(1)}\right)\left(\lambda \gamma^{n} W_{(3)}\right)\left(\lambda \gamma^{p} W_{(4)}\right) k_{[m}^{(2)} \mathcal{F}_{n p]}^{(2)} \\
= & 0
\end{aligned}
$$

where, in the second equality, the momentum conservation and $k W=0$ have been used.

Finally we illustrate with $\left(D_{\beta_{4}} W_{(1)}^{\beta_{1}}\right)\left(D_{\alpha_{1}} W_{(2)}^{\beta_{2}}\right) W_{(3)}^{\beta_{3}}\left(D_{\alpha_{2}} W_{(4)}^{\beta_{4}}\right)$ the computations that involve the terms with all three $D$ 's acting on the different $W^{\prime}$ 's. Using the third eq. of (4), the equation above becomes

$$
\doteq\left(\lambda \gamma^{m_{3} n_{3}} \gamma^{m_{1} n_{1}} \gamma^{m_{2}} \lambda\right)\left(\lambda \gamma^{q} W_{(3)}\right) \mathcal{F}_{m_{1} n_{1}}^{(1)} \mathcal{F}_{m_{2} q}^{(2)} \mathcal{F}_{m_{3} n_{3}}^{(4)}
$$

After a series of manipulations using the identities given in Appendix B and the Fierz rearrangement identities, it becomes the desired expression

$$
\left(\lambda \gamma^{\mu_{1} \ldots \mu_{5}} \lambda\right)\left(\lambda \gamma^{s} W_{(3)}\right) \mathcal{F}_{\mu_{1} \mu_{2}}^{(1)} \mathcal{F}_{\mu_{3} \mu_{4}}^{(4)} \mathcal{F}_{\mu_{5} s}^{(2)}
$$

\section{Three-loop analysis}

The prescription for the three-loop amplitude (the main object of the present work) is given in (10) and quoted here for convenience,

$$
\mathcal{A}_{3-\text { loop }}=\int d \tau_{1} d \cdots d \tau_{6}<\mathcal{N}_{3}(y) \prod_{s=1}^{6} \int d w_{s} \mu\left(w_{s}\right) b\left(w_{s}\right) \int d z_{1} U_{1}\left(z_{1}\right) \cdots \int d z_{N} U_{N}\left(z_{N}\right)>
$$


The two-loop regulator

$$
\mathcal{N}_{2}=e^{-\bar{\lambda} \lambda-r \theta-\bar{w} w+s d}
$$

does not properly carry out the $\lambda \bar{\lambda} \sim 0$ regulation in the case of the three-loop. The six $b$-ghosts produce several potentially divergent terms. Although most of these terms get removed by the $d$-integration, the result of the computation depends on the order of the integrations. Namely, if one performs the $\lambda$-integration prior to the $d$-integration, one gets an ambiguous expression. One possible resolution was discussed by Berkovits and Nekrasov [35]. The idea is to shift $\lambda$ and $\bar{\lambda}$ so that the singularity in $\frac{1}{\lambda \lambda}$ can be avoided. The task becomes more complicated in order to make the shifted spinors satisfy the pure spinor constraints. Elaborating on the idea of [35], Aisaka and Berkovits proposed in [34] a regulator

$$
\mathcal{N}_{3}=\quad \exp \left[-\sum_{I}^{3}\left(\bar{w}_{I}^{\alpha} w_{\alpha, I}+s_{I}^{\alpha} d_{\alpha, I}\right)\right] \exp \left(f^{\alpha} w_{\alpha}+g^{\alpha} d_{\alpha}+\bar{f}_{\alpha} \bar{w}^{\alpha}+\bar{g}_{\alpha} s^{\alpha}\right)
$$

The regulator is not gauge invariant. It was introduced to serve a specific purpose: to show how the regularization works in a simplified setup. In the next section, we take the two-loop regulator, (65), in the three-loop environment and carry out relatively simple computations. The justification for using a two-loop regulator in the three-loop computations will be given in the beginning of that section. In the section that follows, we examine the calculations that involve (66).

A few remarks are in order. Some of the higher power terms in $\frac{1}{\lambda \lambda}$ in the amplitude make the two-loop regulator invalid based on a "power counting". As we will see below, those terms do not contribute to the computations with (66) inserted. Therefore, to the extent that they are genuine regulators it should not matter which regulator to use. This being the case, there is a problem since the computation with the twoloop regulator leads to a vanishing result. Therefore, the complete analysis with (66) inserted could also lead to a vanishing result. Fortunately, this undesirable possibility does not seem to prevent one from acquiring the final forms ${ }^{10}$ of the terms in a given amplitude. This is because of the way the calculations in the pure spinor formulation work. What happens in the two-loop case may be useful for this point. In the twoloop case, the $d$-structures given in (32) yield vanishing results. What causes the vanishing in some cases is pairwise cancelations among different terms. They produce the correct form given in (53) when evaluated in a brute-force type calculation where no attention is paid to the overall numerical coefficient. ${ }^{11}$ It is just that the overall

\footnotetext{
${ }^{10} \mathrm{~A}$ form that is a three-loop analogue of $(53)$ is meant.

${ }^{11}$ A specific example may be helpful. In the section 3.1, eq.(41) was shown to vanish due to the identity given in (42). One could have used the identity (5), instead, in the manner that is similar to that of the section 3.2 where the two-loop amplitude, (53), is derived. By doing so, one amounts to not tracking the overall numerical coefficient. One can show after some algebra the resulting expression is proportional to (53).
} 
numerical coefficient vanishes. We believe that it may well be a general feature of the pure spinor formulation. ${ }^{12}$ In section 4.2 , we select a few representative examples of the $d$-structures. We simplify them to the expressions that are suitable for the further momentum space evaluation, a task that we intend to take in the near future. Strictly speaking, the three-loop amplitude proposed in (103) is not a rigorous result in the sense that not all the contributions are analyzed.

\section{1 warm-up computation}

The complete analysis using (66) is tedious and lengthy. It makes repeated use of a fixed set of techniques. In this section, we display those techniques in a more controlled and less diverse environment by using the two-loop regulator, (65), in the three-loop calculation. There are two main reasons for this. Firstly, the essential part of the three-loop calculation with (65) inserted will re-emerge in the three-loop calculation with (66) inserted. This will become clear in the next section. The second reason is more theoretical. Inserted in the three-loop, the two-loop regulator, (65), does not regulate some of the terms that come in higher powers of $\frac{1}{\lambda \lambda}$ whereas the regulator in (66) does. Howevefr, it regulates them to zero as can be seen by considering the $d$ integration prior to the $\lambda$-integration. Therefore, it should not matter which regulator to use to carry out the integration of the terms of lower powers in $\frac{1}{\lambda \lambda} \cdot{ }^{13}$

For the three-loop, we will need $48 d$-zero modes. Inspection of the integration over the $d$-zero modes reveals that there are three potentially non-zero contributions, ${ }^{14}$

$$
\begin{aligned}
& \int[d \lambda][d \bar{\lambda}][d r] d^{16} \theta \prod_{I=1}^{3}\left[d w^{I}\right]\left[d \bar{w}^{I}\right]\left[d s^{I}\right] d^{16} d^{I} e^{-\bar{\lambda} \lambda-r \theta-\bar{w} w+s d} \\
& {\left[\left(d_{\alpha_{1}} W_{(1)}^{\alpha_{1}}\right)\left(d_{\alpha_{2}} W_{(2)}^{\alpha_{2}}\right)\left(d_{\alpha_{3}} W_{(3)}^{\alpha_{3}}\right)\left(d_{\alpha_{4}} W_{(4)}^{\alpha_{4}}\right)\right.} \\
& +\left(\frac{\left(\bar{\lambda} \gamma^{m n p} r\right)\left(d \gamma_{m n p} d\right)}{192(\bar{\lambda} \lambda)^{2}}\right)^{6}\left(\partial \theta^{\alpha_{1}} A_{\alpha_{1}}+\Pi^{m_{1}} A_{m_{1}}+\frac{1}{2} N^{m_{1} n_{1}} \mathcal{F}_{m_{1} n_{1}}\right) \\
& \left.\quad\left(d_{\alpha_{2}} W_{(2)}^{\alpha_{2}}\right)\left(d_{\alpha_{3}} W_{(3)}^{\alpha_{3}}\right)\left(d_{\alpha_{4}} W_{(4)}^{\alpha_{4}}\right)\right]
\end{aligned}
$$

\footnotetext{
${ }^{12}$ It is a reasonable possibility that the role of a gauge invariant regulator is merely to make the overall coefficient "certainly non-vanishing".

${ }^{13}$ There is a subtlety. The logic assumes that (65) and (66) are genuine regulators. According to [36] they may originate from gauge fixing.

${ }^{14}$ As pointed out by H. Gomez, there could be contributions from$$
\left(\frac{\left(\bar{\lambda} \gamma^{m n p} r\right)\left(d \gamma_{m n p} d\right)}{192(\bar{\lambda} \lambda)^{2}}\right)^{6}\left(d_{\alpha_{1}} W_{(1)}^{\alpha_{1}}\right)\left(d_{\alpha_{2}} W_{(2)}^{\alpha_{2}}\right)\left(d_{\alpha_{3}} W_{(3)}^{\alpha_{3}}\right)\left(d_{\alpha_{4}} W_{(4)}^{\alpha_{4}}\right)
$$

after one of $d$ 's in $\left(d \gamma_{m n p} d\right)^{6}$ gets removed via OPE with one the $W$ 's in $(d W)^{4}$. The analysis of $\left(d \gamma_{m n p} d\right)^{5}$-case in $(68)$ below can be applied to this case with only minimal modifications.
} 


$$
\begin{aligned}
& +\left(\frac{\left(\bar{\lambda} \gamma^{m n p} r\right)\left(d \gamma_{m n p} d\right)}{192(\bar{\lambda} \lambda)^{2}}\right)^{5}\left(\bar{\lambda} \gamma^{m^{\prime}} d\right)\left(\frac{\Pi^{m^{\prime}}}{2(\bar{\lambda} \lambda)}-\frac{\left(r \gamma_{m^{\prime} n^{\prime} p^{\prime}} r\right) N^{n^{\prime} p^{\prime}}}{16(\bar{\lambda} \lambda)^{3}}\right) \\
& \quad\left(d_{\alpha_{1}} W_{(1)}^{\alpha_{1}}\right)\left(d_{\alpha_{2}} W_{(2)}^{\alpha_{2}}\right)\left(d_{\alpha_{3}} W_{(3)}^{\alpha_{3}}\right)\left(d_{\alpha_{4}} W_{(4)}^{\alpha_{4}}\right)
\end{aligned}
$$

The first term in the square bracket is the case where the regulator $\mathcal{N}_{2}$ should provide $44 d$-zero modes. The six $b$-ghosts must not provide any additional $d$-zero modes. The vertex operators provide four of them.

$$
\begin{aligned}
& \int[d \lambda][d \bar{\lambda}][d r] d^{16} \theta \prod_{I=1}^{3}\left[d w^{I}\right]\left[d \bar{w}^{I}\right]\left[d s^{I}\right] d^{16} d^{I} \\
& e^{-\bar{\lambda} \lambda-r \theta-\bar{w} w+s d} \\
& {\left[d_{\alpha_{1}} W^{\alpha_{1}}\left(z_{1}\right)\right]\left[d_{\alpha_{2}} W^{\alpha_{2}}\left(z_{2}\right)\right]\left[d_{\alpha_{3}} W^{\alpha_{3}}\left(z_{3}\right)\right]\left[d_{\alpha_{4}} W^{\alpha_{4}}\left(z_{4}\right)\right]}
\end{aligned}
$$

It is easy to see that this vanishes simply by carrying out the $[d s]$-integration. Some of the vanishing contributions here and below may be seen by a symmetry argument as well that is similar to the one in [25]. For the second term in (68), the six $b$-ghosts should provide 12 remaining $d$ 's and the vertex operators 3 ,

$$
\begin{aligned}
& \int[d \lambda][d \bar{\lambda}][d r] d^{16} \theta \prod_{I=1}^{g}\left[d w^{I}\right]\left[d \bar{w}^{I}\right]\left[d s^{I}\right] d^{16} d^{I} \\
& e^{-\bar{\lambda} \lambda-r \theta-\bar{w} w+s d}\left[\frac{\left(\bar{\lambda} \gamma^{m n p} r\right)\left(d \gamma_{m n p} d\right)}{192(\bar{\lambda} \lambda)^{2}}\right]^{6} \\
& {\left[\partial \theta^{\alpha_{1}} A_{\alpha_{1}}+\Pi^{m_{1}} A_{m_{1}}+\frac{1}{2} N^{m_{1} n_{1}} \mathcal{F}_{m_{1} n_{1}}\right]\left[d_{\alpha_{2}} W^{\alpha_{2}}\left(z_{2}\right)\right]\left[d_{\alpha_{3}} W^{\alpha_{3}}\left(z_{3}\right)\right]\left[d_{\alpha_{4}} W^{\alpha_{4}}\left(z_{4}\right)\right]}
\end{aligned}
$$

Let us focus on the following factors,

$$
\left[\left(\bar{\lambda} \gamma^{m n p} r\right)\left(d \gamma_{m n p} d\right)\right]^{6}
$$

Each factor of $\left(d \gamma_{m n p} d\right)$ consists of several combinations of the zero modes

$$
\begin{aligned}
\left(d \gamma_{m n p} d\right) \doteq \quad & \left(d_{0} \gamma_{m n p} d_{0}\right) \oplus\left(d_{0}^{\prime} \gamma_{m n p} d_{0}^{\prime}\right) \oplus\left(d_{0}^{\prime \prime} \gamma_{m n p} d_{0}^{\prime \prime}\right) \\
& \oplus\left(d_{0} \gamma_{m n p} d_{0}^{\prime}\right) \oplus\left(d_{0} \gamma_{m n p} d_{0}^{\prime \prime}\right) \oplus\left(d_{0}^{\prime} \gamma_{m n p} d_{0}^{\prime \prime}\right)
\end{aligned}
$$

where the zero mode subscripts have been temporarily re-inserted. Let us call the terms in the first line the diagonal terms and the ones in the second line the cross terms,

$$
\begin{aligned}
& \left(d_{0}^{I} \gamma_{m n p} d_{0}^{I}\right): \quad \text { a diogonal term } \\
& \left(d_{0}^{I} \gamma_{m n p} d_{0}^{J}\right), I \neq J: \text { a cross term }
\end{aligned}
$$

Eq.(71) produces several different types of terms depending which factors in (72) are present. Before getting into specific cases, a general structural analysis might be useful. 
Consider (82) and break, into three groups, the expression that results from the $d$ integration:

$$
\begin{aligned}
\text { I : } \quad[ & \left(\lambda \gamma^{r}\right)_{\alpha_{1}}\left(\lambda \gamma^{s}\right)_{\alpha_{2}}\left(\lambda \gamma^{q}\right)_{\alpha_{3}}\left(\gamma_{r s q}\right)_{\alpha_{4} \alpha_{5}} \\
& \left(\lambda \gamma^{r^{\prime}}\right)_{\alpha_{1}^{\prime}}\left(\lambda \gamma^{s^{\prime}}\right)_{\alpha_{2}^{\prime}}\left(\lambda \gamma^{q^{\prime}}\right)_{\alpha_{3}^{\prime}}\left(\gamma_{r^{\prime} s^{\prime} q^{\prime}}\right)_{\alpha_{4}^{\prime} \alpha_{5}^{\prime}} \\
& \left.\left(\lambda \gamma^{r^{\prime \prime}}\right)_{\alpha_{1}^{\prime \prime}}\left(\lambda \gamma^{s^{\prime \prime}}\right)_{\alpha_{2}^{\prime \prime}}\left(\lambda \gamma^{q^{\prime \prime}}\right)_{\alpha_{3}^{\prime \prime}}\left(\gamma_{r^{\prime \prime} s^{\prime \prime} q^{\prime \prime}}\right)_{\alpha_{4}^{\prime \prime} \alpha_{5}^{\prime \prime}}\right], \\
I I: \quad & \left(\gamma_{m_{1} n_{1} p_{1}}\right)^{\kappa_{1} \kappa_{2}}\left(\gamma_{m_{2} n_{2} p_{2}}\right)^{\kappa_{3} \kappa_{4}}\left(\gamma_{m_{3} n_{3} p_{3}}\right)^{\kappa_{5} \kappa_{6}}\left(\gamma_{m_{4} n_{4} p_{4}}\right)^{\kappa_{7} \kappa_{8}}\left(\gamma_{m_{5} n_{5} p_{5}}\right)^{\kappa_{9} \kappa_{10}}\left(\gamma_{m_{6} n_{6} p_{6}}\right)^{\kappa_{11} \kappa_{12}} \\
& \left(\bar{\lambda}_{\sigma_{1}}\left(\gamma^{m_{1} n_{1} p_{1}}\right)^{\sigma_{1} \sigma_{2}} r_{\sigma_{2}}\right)\left(\bar{\lambda}_{\sigma_{3}}\left(\gamma^{m_{2} n_{2} p_{2}}\right)^{\sigma_{3} \sigma_{4}} r_{\sigma_{4}}\right)\left(\bar{\lambda}_{\sigma_{5}}\left(\gamma^{m_{3} n_{3} p_{3}}\right)^{\sigma_{5} \sigma_{6}} r_{\sigma_{6}}\right) \\
& \left(\bar{\lambda}_{\sigma_{7}}\left(\gamma^{m_{4} n_{4} p_{4}}\right)^{\sigma_{7} \sigma_{8}} r_{\sigma_{8}}\right)\left(\bar{\lambda}_{\sigma_{9}}\left(\gamma^{m_{5} n_{5} p_{5}}\right)^{\sigma_{9} \sigma_{10}} r_{\sigma_{10}}\right)\left(\bar{\lambda}_{\sigma_{11}}\left(\gamma^{m_{6} n_{6} p_{6}}\right)^{\sigma_{11} \sigma_{12}} r_{\sigma_{12}}\right), \\
& {\left[W^{\beta_{2}}\left(z_{2}\right)\right]\left[W^{\beta_{3}}\left(z_{3}\right)\right]\left[W^{\beta_{4}}\left(z_{4}\right)\right] }
\end{aligned}
$$

We have suppressed factors of Kronecker deltas that dictate contractions of $(\kappa, \beta)$ indices with $\alpha$-indices. Appling the Fierz identity, (B.4), and using one the pure spinor constraints, $\left(\bar{\lambda} \gamma^{\mu} r\right)=0$, the group (II) becomes

$$
\begin{aligned}
& {\left[\left(r \gamma^{u_{1}}\right)^{\kappa_{1}}\left(\bar{\lambda} \gamma_{u_{1}}\right)^{\kappa_{2}}\right]\left[\left(r \gamma^{u_{2}}\right)^{\kappa_{3}}\left(\bar{\lambda} \gamma_{u_{2}}\right)^{\kappa_{4}}\right]\left[\left(r \gamma^{u_{3}}\right)^{\kappa_{5}}\left(\bar{\lambda} \gamma_{u_{3}}\right)^{\kappa_{6}}\right]} \\
& {\left[\left(r \gamma^{u_{4}}\right)^{\kappa_{7}}\left(\bar{\lambda} \gamma_{u_{4}}\right)^{\kappa_{8}}\right]\left[\left(r \gamma^{u_{5}}\right)^{\kappa_{9}}\left(\bar{\lambda} \gamma_{u_{5}}\right)^{\kappa_{10}}\right]\left[\left(r \gamma^{u_{6}}\right)^{\kappa_{11}}\left(\bar{\lambda} \gamma_{u_{6}}\right)^{\kappa_{12}}\right]}
\end{aligned}
$$

These factors get contracted with the factors in the group (I) in various ways. The resulting expressions allow further simplification by way of the pure spinor constraints and the gamma matrix properties. We illustrate this with a few examples. When there are two or more of the diagonal terms, $\left(d^{I} \gamma_{m n p} d^{I}\right)$ with the given $I$, the contribution trivially vanishes due to the index structure. The following type also vanishes

$$
\left(d^{I} \gamma_{m_{1} n_{1} p_{1}} d^{J}\right)\left(d^{I} \gamma_{m_{2} n_{2} p_{2}} d^{J}\right) \ldots
$$

For example, consider $\left(d \gamma_{m_{1} n_{1} p_{1}} d^{\prime}\right)\left(d \gamma_{m_{2} n_{2} p_{2}} d^{\prime}\right) \cdots$. It implies that (75) contains

$$
\left[\left(r \gamma^{u_{1}}\right)^{\kappa_{1}}\left(\bar{\lambda} \gamma_{u_{1}}\right)^{\kappa_{1}^{\prime}}\right]\left[\left(r \gamma^{u_{2}}\right)^{\kappa_{2}}\left(\bar{\lambda} \gamma_{u_{2}}\right)^{\kappa_{2}^{\prime}}\right]
$$

The contractions with the group (I) factors can be chosen in such a way that the resulting expression contains

$$
\left(r \gamma^{u_{1}} \gamma^{r s q} \gamma^{u_{2}} r\right)\left(\bar{\lambda} \gamma^{u_{1}} \gamma^{r^{\prime} s^{\prime} q^{\prime}} \gamma^{u_{2}} \bar{\lambda}\right)
$$

It vanishes because the first factor is symmetric under $u_{1} \leftrightarrow u_{2}$ while the second factor is anti-symmetric. Therefore only the following form, up to interchanges of roles of $\left(d, d^{\prime}, d^{\prime \prime}\right)$,

$$
\begin{aligned}
& \left(d \gamma_{m_{1} n_{1} p_{1}} d\right)\left(d^{\prime} \gamma_{m_{2} n_{2} p_{2}} d^{\prime}\right)\left(d^{\prime \prime} \gamma_{m_{3} n_{3} p_{3}} d^{\prime \prime}\right)\left(d \gamma_{m_{4} n_{4} p_{4}} d^{\prime}\right)\left(d \gamma_{m_{5} n_{5} p_{5}} d^{\prime \prime}\right)\left(d^{\prime} \gamma_{m_{6} n_{6} p_{6}} d^{\prime \prime}\right) \\
& \left(\bar{\lambda} \gamma^{m_{1} n_{1} p_{1}} r\right)\left(\bar{\lambda} \gamma^{m_{2} n_{2} p_{2}} r\right)\left(\bar{\lambda} \gamma^{m_{3} n_{3} p_{3}} r\right)\left(\bar{\lambda} \gamma^{m_{4} n_{4} p_{4}} r\right)\left(\bar{\lambda} \gamma^{m_{5} n_{5} p_{5}} r\right)\left(\bar{\lambda} \gamma^{m_{6} n_{6} p_{6}} r\right)
\end{aligned}
$$


is potentially non-vanishing. Taking the steps that are similar to those in section 2.2.1, one gets

$$
\begin{aligned}
& \left(r \gamma^{r s q} \bar{\lambda}\right)\left(r \gamma^{r^{\prime} s^{\prime} q^{\prime}} \bar{\lambda}\right)\left(r \gamma^{r^{\prime \prime} s^{\prime \prime} q^{\prime \prime}} \bar{\lambda}\right) \\
& \left(r \gamma^{u_{4}} \gamma^{r} \lambda\right)\left(\bar{\lambda} \gamma_{u_{4}} \gamma^{r^{\prime}} \lambda\right)\left(r \gamma^{u_{5}} \gamma^{s} \lambda\right)\left(\bar{\lambda} \gamma_{u_{5}} \gamma^{r^{\prime}} \lambda\right)\left(r \gamma^{u_{4}} \gamma^{s^{\prime}} \lambda\right)\left(\bar{\lambda} \gamma_{u_{4}} \gamma^{s^{\prime \prime}} \lambda\right) \\
\sim & (\bar{\lambda} \lambda)^{3}(\bar{\lambda} \lambda)\left(r \gamma^{r s q} \bar{\lambda}\right)\left(r \gamma^{r^{\prime} s^{\prime} q^{\prime}} \bar{\lambda}\right)\left(r \gamma^{r^{\prime \prime} s^{\prime \prime} q^{\prime \prime}} \bar{\lambda}\right) \\
& \left(r \gamma^{r^{\prime}} \gamma^{r} \lambda\right)\left(r \gamma^{r^{\prime \prime}} \gamma^{s} \lambda\right)\left(r \gamma^{s^{\prime \prime}} \gamma^{s^{\prime}} \lambda\right)
\end{aligned}
$$

As in section 2.2.1, the factor, $\left(r \gamma^{r^{\prime}} \gamma^{r} \lambda\right)$ can be replaced by $\eta^{r^{\prime} r}(\lambda r)$ and the expression vanishes. The third term in (68) is where the regulator $\mathcal{N}_{2}$ provides $33 d$-zero modes. The six $b$-ghosts should provide 11 remaining $d$ 's and the vertex operators 4 . One gets

$$
\begin{aligned}
& \int[d \lambda][d \bar{\lambda}][d r] d^{16} \theta \prod_{I=1}^{3}\left[d w^{I}\right]\left[d \bar{w}^{I}\right]\left[d s^{I}\right] d^{16} d^{I} \\
& e^{-\bar{\lambda} \lambda-r \theta-\bar{w} w+s d} \\
& {\left[\frac{\left(\bar{\lambda} \gamma^{m n p} r\right)\left(d \gamma_{m n p} d\right)}{192(\bar{\lambda} \lambda)^{2}}\right]^{5}\left(\frac{\Pi^{m^{\prime}}\left(\bar{\lambda} \gamma_{m^{\prime}} d\right)}{2(\bar{\lambda} \lambda)}-\frac{\left(r \gamma_{m^{\prime} n^{\prime} p^{\prime}} r\right)\left(\bar{\lambda} \gamma^{m^{\prime}} d\right) N^{n^{\prime} p^{\prime}}}{16(\bar{\lambda} \lambda)^{3}}\right) } \\
\sim \quad & {\left[d_{\alpha_{1}} W^{\alpha_{1}}\left(z_{1}\right)\right]\left[d_{\alpha_{2}} W^{\alpha_{2}}\left(z_{2}\right)\right]\left[d_{\alpha_{3}} W^{\alpha_{3}}\left(z_{3}\right)\right]\left[d_{\alpha_{4}} W^{\alpha_{4}}\left(z_{4}\right)\right] } \\
& \left.\int d \lambda\right][d \bar{\lambda}][d r] d^{16} \theta \prod_{I=1}^{3}\left[d w^{I}\right]\left[d \bar{w}^{I}\right]\left[d s^{I}\right] d^{16} d^{I} \\
& e^{-\bar{\lambda} \lambda-r \theta-\bar{w} w}(s d)^{33} \\
& {\left[\frac{\left(\bar{\lambda} \gamma^{m n p} r\right)\left(d \gamma_{m n p} d\right)}{192(\bar{\lambda} \lambda)^{2}}\right]^{5}\left(\bar{\lambda} \gamma^{m^{\prime}} d\right)\left(\frac{\Pi^{m^{\prime}}}{2(\bar{\lambda} \lambda)}-\frac{\left(r \gamma_{m^{\prime} n^{\prime} p^{\prime}} r\right) N^{n^{\prime} p^{\prime}}}{16(\bar{\lambda} \lambda)^{3}}\right) } \\
& {\left[d_{\alpha_{1}} W^{\alpha_{1}}\left(z_{1}\right)\right]\left[d_{\alpha_{2}} W^{\alpha_{2}}\left(z_{2}\right)\right]\left[d_{\alpha_{3}} W^{\alpha_{3}}\left(z_{3}\right)\right]\left[d_{\alpha_{4}} W^{\alpha_{4}}\left(z_{4}\right)\right] }
\end{aligned}
$$

Performing $s$-integration, one gets

$$
\begin{aligned}
\sim & \int[d \lambda][d \bar{\lambda}][d r] d^{16} \theta \prod_{I=1}^{3}\left[d w^{I}\right]\left[d \bar{w}^{I}\right] d^{16} d^{I} \\
& {\left[\left(\lambda \gamma^{r}\right)_{\alpha_{1}}\left(\lambda \gamma^{s}\right)_{\alpha_{2}}\left(\lambda \gamma^{q}\right)_{\alpha_{3}}\left(\gamma_{r s q}\right)_{\alpha_{4} \alpha_{5}} \epsilon^{\alpha_{1} \ldots \alpha_{5} \rho_{1} \ldots \rho_{11}} d_{\rho_{1} \ldots d_{\rho_{11}}}\right.} \\
& \left(\lambda \gamma^{r^{\prime}}\right)_{\alpha_{1}^{\prime}}\left(\lambda \gamma^{s^{\prime}}\right)_{\alpha_{2}^{\prime}}\left(\lambda \gamma^{q^{\prime}}\right)_{\alpha_{3}^{\prime}}\left(\gamma_{r^{\prime} s^{\prime} q^{\prime}}\right)_{\alpha_{4}^{\prime} \alpha_{5}^{\prime}} \epsilon^{\alpha_{1}^{\prime} \ldots \alpha_{5}^{\prime} \rho_{1}^{\prime} \ldots \rho_{11}^{\prime}} d_{\rho_{1}^{\prime} \ldots d_{\rho_{11}^{\prime}}^{\prime}} \\
& \left.\left(\lambda \gamma^{r^{\prime \prime}}\right)_{\alpha_{1}^{\prime \prime}}\left(\lambda \gamma^{s^{\prime \prime}}\right)_{\alpha_{2}^{\prime \prime}}\left(\lambda \gamma^{q^{\prime \prime}}\right)_{\alpha_{3}^{\prime \prime}}\left(\gamma_{r^{\prime \prime} s^{\prime \prime} q^{\prime \prime}}\right)_{\alpha_{4}^{\prime \prime} \alpha_{5}^{\prime \prime}} \epsilon_{1}^{\alpha_{1}^{\prime \prime} \ldots \alpha_{5}^{\prime \prime} \rho_{1}^{\prime \prime} \ldots \rho_{11}^{\prime \prime}} d_{\rho_{1}^{\prime \prime} \ldots}^{\prime \prime} d_{\rho_{11}^{\prime \prime}}^{\prime \prime}\right] \\
& {\left[\frac{\left(\bar{\lambda} \gamma^{m n p} r\right)\left(d \gamma_{m n p} d\right)}{192(\bar{\lambda} \lambda)^{2}}\right]^{5}\left(\bar{\lambda} \gamma^{m^{\prime}} d\right)\left(\frac{\Pi^{m^{\prime}}}{2(\bar{\lambda} \lambda)}-\frac{\left(r \gamma_{m^{\prime} n^{\prime} p^{\prime}} r\right) N^{n^{\prime} p^{\prime}}}{16(\bar{\lambda} \lambda)^{3}}\right) } \\
& {\left[d_{\beta_{1}} W^{\beta_{1}}\left(z_{1}\right)\right]\left[d_{\beta_{2}} W^{\beta_{2}}\left(z_{2}\right)\right]\left[d_{\beta_{3}} W^{\beta_{3}}\left(z_{3}\right)\right]\left[d_{\beta_{4}} W^{\beta_{4}}\left(z_{4}\right)\right] }
\end{aligned}
$$

Let us focus on the following factors in (82),

$$
\left[\left(\bar{\lambda} \gamma^{m n p} r\right)\left(d \gamma_{m n p} d\right)\right]^{5}\left(\bar{\lambda} \gamma^{m^{\prime}} d\right)
$$


With the experience gained in the analysis of $\left(d \gamma^{(3)} d\right)^{6}$ above, it is not difficult to see that the only potentially non-vanishing forms are

$$
\begin{aligned}
& \left(d \gamma_{m_{1} n_{1} p_{1}} d\right)\left(d^{\prime} \gamma_{m_{2} n_{2} p_{2}} d^{\prime}\right)\left(d \gamma_{m_{3} n_{3} p_{3}} d^{\prime}\right)\left(d \gamma_{m_{4} n_{4} p_{4}} d^{\prime \prime}\right)\left(d^{\prime} \gamma_{m_{5} n_{5} p_{5}} d^{\prime \prime}\right) \\
& \left(d \gamma_{m_{1} n_{1} p_{1}} d\right)\left(d^{\prime} \gamma_{m_{2} n_{2} p_{2}} d^{\prime}\right)\left(d^{\prime \prime} \gamma_{m_{3} n_{3} p_{3}} d^{\prime \prime}\right)\left(d \gamma_{m_{4} n_{4} p_{4}} d^{\prime}\right)\left(d \gamma_{m_{5} n_{5} p_{5}} d^{\prime \prime}\right)
\end{aligned}
$$

and the ones with $\left(d, d^{\prime}, d^{\prime \prime}\right)$ interchanged. As we illustrate below, these terms vanish. Put (82) into three groups,

$$
\begin{aligned}
\text { I : } \quad[ & \left(\lambda \gamma^{r}\right)_{\alpha_{1}}\left(\lambda \gamma^{s}\right)_{\alpha_{2}}\left(\lambda \gamma^{q}\right)_{\alpha_{3}}\left(\gamma_{r s q}\right)_{\alpha_{4} \alpha_{5}} \\
& \left(\lambda \gamma^{r^{\prime}}\right)_{\alpha_{1}^{\prime}}\left(\lambda \gamma^{s^{\prime}}\right)_{\alpha_{2}^{\prime}}\left(\lambda \gamma^{q^{\prime}}\right)_{\alpha_{3}^{\prime}}\left(\gamma_{r^{\prime} s^{\prime} q^{\prime}}\right)_{\alpha_{4}^{\prime} \alpha_{5}^{\prime}} \\
& \left.\left(\lambda \gamma^{r^{\prime \prime}}\right)_{\alpha_{1}^{\prime \prime}}\left(\lambda \gamma^{s^{\prime \prime}}\right)_{\alpha_{2}^{\prime \prime}}\left(\lambda \gamma^{q^{\prime \prime}}\right)_{\alpha_{3}^{\prime \prime}}\left(\gamma_{r^{\prime \prime} s^{\prime \prime} q^{\prime \prime}}\right)_{\alpha_{4}^{\prime \prime} \alpha_{5}^{\prime \prime}}\right], \\
I I: \quad & \left(\gamma_{m_{1} n_{1} p_{1}}\right)^{\kappa_{1} \kappa_{2}}\left(\gamma_{m_{2} n_{2} p_{2}}\right)^{\kappa_{3} \kappa_{4}}\left(\gamma_{m_{3} n_{3} p_{3}}\right)^{\kappa_{5} \kappa_{6}}\left(\gamma_{m_{4} n_{4} p_{4}}\right)^{\kappa_{7} \kappa_{8}}\left(\gamma_{m_{5} n_{5} p_{5}}\right)^{\kappa_{9} \kappa_{10}} \\
& \left(\bar{\lambda}_{\sigma_{1}}\left(\gamma^{m_{1} n_{1} p_{1}}\right)^{\sigma_{1} \sigma_{2}} r_{\sigma_{2}}\right)\left(\bar{\lambda}_{\sigma_{3}}\left(\gamma^{m_{2} n_{2} p_{2}}\right)^{\sigma_{3} \sigma_{4}} r_{\sigma_{4}}\right)\left(\bar{\lambda}_{\sigma_{5}}\left(\gamma^{m_{3} n_{3} p_{3}}\right)^{\sigma_{5} \sigma_{6}} r_{\sigma_{6}}\right) \\
& \left(\bar{\lambda}_{\sigma_{7}}\left(\gamma^{m_{4} n_{4} p_{4}}\right)^{\sigma_{7} \sigma_{8}} r_{\sigma_{8}}\right)\left(\bar{\lambda}_{\sigma_{9}}\left(\gamma^{m_{5} n_{5} p_{5}}\right)^{\sigma_{9} \sigma_{10}} r_{\sigma_{10}}\right), \\
I I I: \quad & \left(\bar{\lambda} \gamma^{m^{\prime}}\right)^{\beta}\left[W^{\beta_{1}}\left(z_{1}\right)\right]\left[W^{\beta_{2}}\left(z_{2}\right)\right]\left[W^{\beta_{3}}\left(z_{3}\right)\right]\left[W^{\beta_{4}}\left(z_{4}\right)\right]
\end{aligned}
$$

Let us take the first term of (84),

$$
\left(d \gamma_{m_{1} n_{1} p_{1}} d\right)\left(d^{\prime} \gamma_{m_{2} n_{2} p_{2}} d^{\prime}\right)\left(d \gamma_{m_{3} n_{3} p_{3}} d^{\prime}\right)\left(d \gamma_{m_{4} n_{4} p_{4}} d^{\prime \prime}\right)\left(d^{\prime} \gamma_{m_{5} n_{5} p_{5}} d^{\prime \prime}\right)
$$

The steps are similar to the case of the $\left(d \gamma^{(3)} d\right)^{6}$. After the $[d s]-,[d d]$ - integrations and Fierzing, one gets an expression that contains

$$
\begin{aligned}
&\left(r \gamma^{r s q} \bar{\lambda}\right)\left(r \gamma^{r^{\prime} s^{\prime} q^{\prime}} \bar{\lambda}\right)\left(r \gamma^{u_{3}} \gamma^{r} \lambda\right)\left(\bar{\lambda} \gamma_{u_{3}} \gamma^{r^{\prime}} \lambda\right) \\
& \sim \quad(\bar{\lambda} \lambda)\left(r \gamma^{r s q} \bar{\lambda}\right)\left(r \gamma^{r^{\prime} s^{\prime} q^{\prime}} \bar{\lambda}\right)\left(r \gamma^{r^{\prime}} \gamma^{r} \lambda\right)
\end{aligned}
$$

The last factor yields $\eta^{r^{\prime} r}$ rendering the expression to vanish.

\subsection{Aisaka-Berkovits regulator}

In the previous section, we have exhibited all the techniques that we will use in this section. A proper regularization of the $\frac{1}{\lambda \lambda}$-singularities for three- and higher- loops was outlined in [35]. The resulting formula is complex. Setting apart the gauge invariance issue, Aisaka and Berkovits have proposed a relatively simple regulator that is quoted in (66). We take (66) and work out a few contributions in detail.

First we survey the amount of terms that need to be computed. One simplifying feature is that the $d$ - and $[d s]$ - integrations should remove many of the terms, if not the majority. Based on inspection of the $d$-integration, one concludes that only the terms 
in the square bracket of (68) are potentially non-zero. In particular we narrow down to the last two terms. ${ }^{15}$ The zero mode integrations now become

$$
\begin{aligned}
& \sim \quad \int[d \lambda][d \bar{\lambda}][d r] d^{16} \theta \prod_{I=1}^{3}\left[d w^{I}\right]\left[d \bar{w}^{I}\right] d^{16} d^{I} \exp \left[-\sum_{I}^{g}\left(\bar{w}_{I}^{\alpha} w_{\alpha, I}\right)\right] \exp \left(f^{\alpha} w_{\alpha}+g^{\alpha} d_{\alpha}+\bar{f}_{\alpha} \bar{w}^{\alpha}\right) \\
& {\left[\left(\lambda \gamma^{r}\right)_{\alpha_{1}}\left(\lambda \gamma^{s}\right)_{\alpha_{2}}\left(\lambda \gamma^{q}\right)_{\alpha_{3}}\left(\gamma_{r s q}\right)_{\alpha_{4} \alpha_{5}} \epsilon^{\alpha_{1} \ldots \alpha_{5} \rho_{1} \ldots \rho_{11}}\left(d_{\rho_{1}}-\bar{g}_{\rho_{1}}\right) \ldots\left(d_{\rho_{11}}-\bar{g}_{\rho_{11}}\right)\right.} \\
& \left(\lambda \gamma^{r^{\prime}}\right)_{\alpha_{1}^{\prime}}\left(\lambda \gamma^{s^{\prime}}\right)_{\alpha_{2}^{\prime}}\left(\lambda \gamma^{q^{\prime}}\right)_{\alpha_{3}^{\prime}}\left(\gamma_{r^{\prime} s^{\prime} q^{\prime}}\right)_{\alpha_{4}^{\prime} \alpha_{5}^{\prime}} \epsilon^{\alpha_{1}^{\prime} \ldots \alpha_{5}^{\prime} \rho_{1}^{\prime} \ldots \rho_{11}^{\prime}}\left(d_{\rho_{1}^{\prime}}^{\prime}-\bar{g}_{\rho_{1}^{\prime}}\right) \ldots\left(d_{\rho_{11}^{\prime}}^{\prime}-\bar{g}_{\rho_{11}^{\prime}}\right) \\
& \left.\left(\lambda \gamma^{r^{\prime \prime}}\right)_{\alpha_{1}^{\prime \prime}}\left(\lambda \gamma^{s^{\prime \prime}}\right)_{\alpha_{2}^{\prime \prime}}\left(\lambda \gamma^{q^{\prime \prime}}\right)_{\alpha_{3}^{\prime \prime}}\left(\gamma_{r^{\prime \prime} s^{\prime \prime} q^{\prime \prime}}\right)_{\alpha_{4}^{\prime \prime} \alpha_{5}^{\prime \prime}} \epsilon^{\alpha_{1}^{\prime \prime} \ldots \alpha_{5}^{\prime \prime} \rho_{1}^{\prime \prime} \ldots \rho_{11}^{\prime \prime}}\left(d_{\rho_{1}^{\prime \prime}}^{\prime \prime}-\bar{g}_{\rho_{1}^{\prime \prime}}\right) \ldots\left(d_{\rho_{11}^{\prime \prime}}^{\prime \prime}-\bar{g}_{\rho_{11}^{\prime \prime}}\right)\right] \\
& \left\{\left[\frac{\left(\bar{\lambda} \gamma^{m n p} r\right)\left(d \gamma_{m n p} d\right)}{192(\bar{\lambda} \lambda)^{2}}\right]^{5}\left(\bar{\lambda} \gamma_{m^{\prime}} d\right)\left(\frac{\Pi^{m^{\prime}}}{2(\bar{\lambda} \lambda)}-\frac{\left(r \gamma_{m^{\prime} n^{\prime} p^{\prime}} r\right) N^{n^{\prime} p^{\prime}}}{16(\bar{\lambda} \lambda)^{3}}\right)\right. \\
& {\left[d_{\alpha_{1}} W^{\alpha_{1}}\left(z_{1}\right)\right]\left[d_{\alpha_{2}} W^{\alpha_{2}}\left(z_{2}\right)\right]\left[d_{\alpha_{3}} W^{\alpha_{3}}\left(z_{3}\right)\right]\left[d_{\alpha_{4}} W^{\alpha_{4}}\left(z_{4}\right)\right]} \\
& +\left[\frac{\left(\bar{\lambda} \gamma^{m n p} r\right)\left(d \gamma_{m n p} d\right)}{192(\bar{\lambda} \lambda)^{2}}\right]^{6}\left[\partial \theta^{\alpha_{1}} A_{\alpha_{1}}+\Pi^{m_{1}} A_{m_{1}}+\frac{1}{2} N^{m_{1} n_{1}} \mathcal{F}_{m_{1} n_{1}}\right] \\
& \left.\left[d_{\alpha_{2}} W^{\alpha_{2}}\left(z_{2}\right)\right]\left[d_{\alpha_{3}} W^{\alpha_{3}}\left(z_{3}\right)\right]\left[d_{\alpha_{4}} W^{\alpha_{4}}\left(z_{4}\right)\right]\right\}
\end{aligned}
$$

Since $g^{\alpha}$ and $\bar{g}_{\alpha}$ are grassmanian, there can be maximum eleven factors for each. Also since the measure contains $d^{11} g, d^{11} \bar{g}$, there must be precisely eleven factors of $g^{\alpha}, \bar{g}_{\alpha}$ respectively. The break-up of the contributions goes as follows. The factor, $\exp \left(g^{\alpha} d_{\alpha}\right)$, contributes eleven $d$-zero modes. The square bracket contributes twenty two $d$-zero modes (and eleven $\bar{g}$ 's). The last two factors then must provide the remaining fifteen. The factor of $\exp \left(g^{\alpha} d_{\alpha}\right)$ and the square bracket will produce terms of the form

$$
\begin{aligned}
& \left(\bar{g}_{\rho_{1}} \ldots \bar{g}_{\rho_{m}} d_{\rho_{m+1}} \ldots d_{\rho_{11}}\right)\left(g^{\sigma_{1}} \ldots g^{\sigma_{M}} d_{\sigma_{1}} \ldots d_{\sigma_{M}}\right) \\
& \left(\bar{g}_{\rho_{1}^{\prime}} \ldots \bar{g}_{\rho_{n}^{\prime}} d_{\rho_{n+1}^{\prime}}^{\prime} \ldots d_{\rho_{11}^{\prime}}^{\prime}\right)\left(g^{\sigma_{1}^{\prime}} \ldots g^{\sigma_{N}^{\prime}} d_{\sigma_{1}^{\prime}}^{\prime} \ldots d_{\sigma_{N}^{\prime}}^{\prime}\right) \\
& \left(\bar{g}_{\rho_{1}^{\prime \prime} \ldots} \ldots \bar{g}_{\rho_{l}^{\prime \prime}} d_{\rho_{l+1}^{\prime \prime}}^{\prime \prime} \ldots d_{\rho_{11}^{\prime \prime}}^{\prime \prime}\right)\left(g^{\sigma_{1}^{\prime \prime}} \ldots g^{\sigma_{L}^{\prime \prime}} d_{\sigma_{1}^{\prime \prime}}^{\prime \prime} \ldots d_{\sigma_{L}^{\prime \prime}}^{\prime \prime}\right)
\end{aligned}
$$

with $m+n+l=11$ and $M+N+L=11$ as can be ssen by considering the number of $\bar{g}$ 's. Let us follow the following line of logic. Suppose that (89) contains less than eleven factors for some of $\left(d, d^{\prime}, d^{\prime \prime}\right)$. The deficit must be compensated by the terms in the square bracket in (88). The integrations over $d g$ and $d \bar{g}$ are carried out with the following Lorentz invariant measures,

$$
\begin{aligned}
& {[d g]=\frac{1}{(\lambda \bar{\lambda})^{3}}\left(\lambda \gamma^{m}\right)_{\alpha_{1}}\left(\lambda \gamma^{n}\right)_{\alpha_{2}}\left(\lambda \gamma^{p}\right)_{\alpha_{3}}\left(\gamma_{m n p}\right)_{\alpha_{4} \alpha_{5}} \epsilon^{\alpha_{1} \ldots \alpha_{5} \delta_{1} \ldots \delta_{11}} \partial_{\delta_{1}}^{g} \ldots \partial_{\delta_{11}}^{g}} \\
& {[d \bar{g}]\left(\lambda \gamma^{m}\right)_{\alpha_{1}}\left(\lambda \gamma^{n}\right)_{\alpha_{2}}\left(\lambda \gamma^{p}\right)_{\alpha_{3}}\left(\gamma_{m n p}\right)_{\alpha_{4} \alpha_{5}}=(\lambda \bar{\lambda})^{3} \epsilon_{\alpha_{1} \ldots \alpha_{5} \delta_{1} \ldots \delta_{11}} \partial_{\bar{g}}^{\delta_{1}} \ldots \partial_{\bar{g}}^{\delta_{11}}}
\end{aligned}
$$

\footnotetext{
${ }^{15}$ In addition, there are the extra contributions that have been indicated in one of the footnotes in section 3.1 .
} 
After carrying out the $g$-integration, one gets, among other factors,

$$
\begin{aligned}
& \epsilon^{\alpha_{1} \ldots \alpha_{5} \rho_{1} \ldots \rho_{11}} \epsilon^{\alpha_{1}^{\prime} \ldots \alpha_{5}^{\prime} \rho_{1}^{\prime} \ldots \rho_{11}^{\prime}} \epsilon^{\alpha_{1}^{\prime \prime} \ldots \alpha_{5}^{\prime \prime} \rho_{1}^{\prime \prime} \ldots \rho_{11}^{\prime \prime}} \quad \delta_{\sigma_{1} \ldots \sigma_{M} \rho_{1}^{\prime} \ldots \rho_{n}^{\prime} \rho_{1}^{\prime \prime} \ldots \sigma_{1}^{\prime \prime} \ldots \sigma_{L}^{\prime \prime}}^{\rho_{1} \ldots \rho_{m}^{\prime}} \\
& \left(d_{\rho_{m+1}} \ldots d_{\rho_{11}} d_{\sigma_{1} \ldots} \ldots d_{\sigma_{M}}\right)\left(d_{\rho_{n+1}^{\prime}}^{\prime} \ldots d_{\rho_{11}^{\prime}}^{\prime} d_{\sigma_{1}^{\prime} \ldots}^{\prime} \ldots d_{\sigma_{N}^{\prime}}^{\prime}\right)\left(d_{\rho_{l+1}^{\prime \prime}}^{\prime \prime} \ldots d_{\rho_{11}^{\prime \prime}}^{\prime \prime \prime} d_{\sigma_{1}^{\prime \prime}}^{\prime \prime} \ldots d_{\sigma_{L}^{\prime \prime}}^{\prime \prime}\right)
\end{aligned}
$$

where the $\epsilon$ 's in the first line come from the $[d s]$-measure. Because of the grassmanian nature of the $\left(d, d^{\prime}, d^{\prime \prime}\right)$ which are dummy variables, the permutation in $\delta_{\sigma_{1} \ldots \sigma_{M} \sigma_{1}^{\prime} \ldots \sigma_{N}^{\prime} \sigma_{1}^{\prime \prime} \ldots \sigma_{L}^{\prime \prime}}^{\rho_{1} \ldots \rho^{\prime} \rho^{\prime \prime} \ldots \rho_{\rho^{\prime \prime}}^{\prime \prime}}$ can be entirely dropped. In all subsequent calculations we only consider

$$
\delta_{\sigma_{1}}^{\rho_{1}} \delta_{\sigma_{2}}^{\rho_{2}} \cdots \delta_{\sigma_{L}^{\prime \prime}}^{\rho_{l \prime \prime}^{\prime \prime}}
$$

i.e., the term that preserves the exact order of the upper and the lower indices as

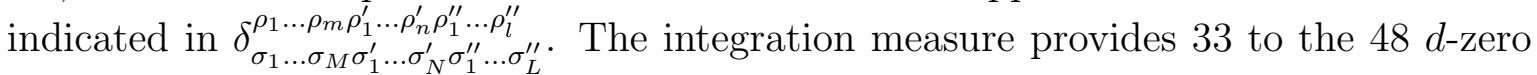
modes needed. We take the first term in (88), which contains $\left(d \gamma^{(3)} d\right)^{5}$, for detailed analysis. The $d_{0}^{I}$-deficit must be compensated for by the factors of the same kind in

$$
\begin{aligned}
& {\left[\frac{\left(\bar{\lambda} \gamma^{m n p} r\right)\left(d \gamma_{m n p} d\right)}{192(\bar{\lambda} \lambda)^{2}}\right]^{5}\left(\bar{\lambda} \gamma_{m^{\prime}} d\right)\left(\frac{\Pi^{m^{\prime}}}{2(\bar{\lambda} \lambda)}-\frac{\left(r \gamma_{m^{\prime} n^{\prime} p^{\prime}} r\right) N^{n^{\prime} p^{\prime}}}{16(\bar{\lambda} \lambda)^{3}}\right)} \\
& {\left[d_{\alpha_{1}} W^{\alpha_{1}}\left(z_{1}\right)\right]\left[d_{\alpha_{2}} W^{\alpha_{2}}\left(z_{2}\right)\right]\left[d_{\alpha_{3}} W^{\alpha_{3}}\left(z_{3}\right)\right]\left[d_{\alpha_{4}} W^{\alpha_{4}}\left(z_{4}\right)\right]}
\end{aligned}
$$

Depending on how the fifteen deficit $d$ 's are distributed, the terms that arrive by expanding $(d \gamma d)^{5}$ can be classified as

$$
\begin{aligned}
\left(n_{d}, n_{d^{\prime}}, n_{d^{\prime \prime}}\right)= & (15,0,0),(14,1,0),(13,2,0),(13,1,1),(12,3,0),(12,2,1),(11,4,0), \\
& (11,3,1),(11,2,2),(10,5,0),(10,4,1),(10,3,2),(9,6,0),(9,5,1), \\
& (9,4,2),(9,3,3),(8,7,0),(8,6,1),(8,5,2),(8,4,3),(7,7,1), \\
& (7,6,2),(7,5,3),(7,4,4),(6,6,3),(6,5,4),(5,5,5)
\end{aligned}
$$

where we have assumed, $n_{d} \geq n_{d^{\prime}} \geq n_{d^{\prime \prime}}$, without loss of generality. It is because the cases otherwise will only change the irrelevant overall factors. The cases with $n_{d}=15, \ldots, 12$ trivially vanish for the following reason. In such cases, the expressions contain

$$
\epsilon_{\ldots \kappa_{1} \kappa_{2} \kappa_{3} \kappa_{4} \ldots}\left(\gamma_{m_{1} n_{1} p_{1}}\right)^{\kappa_{1} \kappa_{2}}\left(\bar{\lambda} \gamma^{m_{1} n_{1} p_{1}} r\right)\left(\gamma_{m_{2} n_{2} p_{2}}\right)^{\kappa_{3} \kappa_{3}}\left(\bar{\lambda} \gamma^{m_{2} n_{2} p_{2}} r\right)
$$

This equation identically vanishes as one can see by interchanging $\left(\gamma_{m_{1} n_{1} p_{1}}\right)^{\kappa_{1} \kappa_{2}}\left(\bar{\lambda} \gamma^{m_{1} n_{1} p_{1}} r\right)$ and $\left(\gamma_{m_{2} n_{2} p_{2}}\right)^{\kappa_{3} \kappa_{3}}\left(\bar{\lambda} \gamma^{m_{2} n_{2} p_{2}} r\right)$. Also the term, $\left(n_{d}, n_{d^{\prime}}, n_{d^{\prime \prime}}\right)=(5,5,5)$, becomes essentially the same as the case considered in the previous section. Therefore the terms that require more careful inspection are

$$
\begin{aligned}
\left(n_{d}, n_{d^{\prime}}, n_{d^{\prime \prime}}\right)= & (11,4,0),(11,3,1),(11,2,2),(10,5,0),(10,4,1),(10,3,2),(9,6,0), \\
& (9,5,1),(9,4,2),(9,3,3),(8,7,0),(8,6,1),(8,5,2),(8,4,3) \\
& (7,7,1),(7,6,2),(7,5,3),(7,4,4),(6,6,3),(6,5,4)
\end{aligned}
$$


Each of these terms has sub-cases depending on how $\left(d, d^{\prime}, d^{\prime \prime}\right)$ is distributed over the factors that come from the vertex operators, $\left(\bar{\lambda} \gamma^{m^{\prime}} d\right)(d W)(d W)(d W)(d W)$.

We now select a few example and maximally simplify them. After the $[d s]$ - and $[d d]$ integrations, a given contribution becomes an equation where the variables, $(\lambda$ 's, $\bar{\lambda}$ 's, $r$ 's, $\gamma^{m_{1} \ldots m_{j}}$ 's) get contracted among themselves and/or with the SYM fields. It gets simplified by applying the Fierz identities and the pure spinor constraints. Often in the middle of manipulations, one encounters the following form of factors,

$$
\left(\ldots \gamma^{p} \gamma^{m} r\right)\left(\ldots \gamma_{p} r\right)
$$

Applying a gamma matrix identity it becomes,

$$
\left(\chi \gamma^{p} \gamma^{m} r\right)\left(\xi \gamma_{p} r\right)=2(\chi r)\left(\xi \gamma^{m} r\right)-\left(\chi^{\prime} \gamma^{p} r\right)\left(\xi \gamma_{p} r\right)
$$

where $\chi^{\prime} \equiv \chi \gamma^{m}$. In the examples below, we focus on the contributions coming from the first part. One of the reasons, which we elaborate on in the Appendix $\mathrm{C}$ and a footnote below, is that the contributions associated with the second term tend to vanish for one reason or another. In the approach that we are taking to simplify, it has something to with the fact that the expression,

$$
\left(\chi^{\prime} \gamma^{\mu \nu \rho} \xi\right)\left(r \gamma_{\mu \nu \rho} r\right)
$$

which results from applying one of the Fierz identity, does not admit the obvious inverse Fierzing because of the changed index structure. One can force the inverse Fierzing but then procedure introduces $\gamma^{0}$ 's. As a matter of fact, what we amount to show in the appendix is that all the structures that do not contain a single factor of $\left(\chi^{\prime} \gamma^{p} r\right)\left(\xi \gamma_{p} r\right)$ drop out due to various constraints and identities.

One of the differences between the two-loop regulator, (65), and the three-loop regulator, (66), is that the three-loop regulator introduces more mixing between terms through (89). Comparison of the cases of $\left(n_{d}, n_{d^{\prime}}, n_{d^{\prime \prime}}\right)=(5,5,5)$ and $\left(n_{d}, n_{d^{\prime}}, n_{d^{\prime \prime}}\right)=$ $(6,5,4)$ may illustrate the point. The case, $(5,5,5)$, is essentially the same as the case that has been analyzed in the previous section. When the $\alpha$-indices in (85) get contract with the $\kappa$-indices, the indices of different numbers of primes are not allowed to contract. It is partially allowed in the latter case because (91) takes the form of

$$
\delta_{\kappa_{1} \ldots \ldots \ldots \kappa_{6}}^{\alpha_{1} \ldots \alpha_{5} \rho_{m}} \quad \delta_{\kappa_{1}^{\prime} \ldots \kappa_{5}^{\prime} \rho_{m}}^{\alpha_{1}^{\prime} \ldots \alpha_{m}^{\prime} \rho_{n}^{\prime}} \delta_{\kappa_{1}^{\prime \prime} \ldots \kappa_{4}^{\prime \prime} \rho_{n}^{\prime}}^{\alpha_{1 \prime \prime}^{\prime \prime} \ldots \alpha_{1}^{\prime \prime}}
$$

The cases listed in (95) allow various degrees of mixing. Once there is a sufficient amount of mixing, the contribution generates the same final result although the terms with different level of mixing produce different looking expressions in the intermediate steps. For the $\left(d \gamma^{(3)} d\right)^{5}$ case

$$
\begin{aligned}
& {\left[\left(\bar{\lambda} \gamma^{m n p} r\right)\left(d \gamma_{m n p} d\right)\right]^{5}} \\
& {\left[d_{\alpha_{1}} W^{\alpha_{1}}\left(z_{1}\right)\right]\left[d_{\alpha_{2}} W^{\alpha_{2}}\left(z_{2}\right)\right]\left[d_{\alpha_{3}} W^{\alpha_{3}}\left(z_{3}\right)\right]\left[d_{\alpha_{4}} W^{\alpha_{4}}\left(z_{4}\right)\right]}
\end{aligned}
$$


Eq.(100) comes multiplied with $\left(\bar{\lambda} \gamma^{m^{\prime}} d\right)\left(r \gamma_{m^{\prime} n^{\prime} p^{\prime}} r\right) N^{n^{\prime} p^{\prime}}$ which can rewritten as

$$
-8\left(r \gamma_{m^{\prime}}\right)(r \lambda)+4\left(r \gamma_{m^{\prime}} \gamma^{\mu} \lambda\right)\left(r \gamma_{\mu} w\right)
$$

after use of one of the Fierz identity. Because the (103) contains one factor of $(\lambda r)$, eq.(101) reduces to

$$
-4\left(r \gamma^{\mu} \gamma_{m^{\prime}} \lambda\right)\left(r \gamma_{\mu} w\right)
$$

We have worked out several exemplary and random cases $^{16}$ and found that they all reduce the following form

$$
(\lambda r)\left(\lambda \gamma^{t_{1}} \gamma^{t_{2}} r\right)[r W]\left[\lambda \gamma^{t_{3}} W\right]\left[r \gamma^{t_{1}} \gamma^{m^{\prime}} W\right]\left[r \gamma^{t_{2}} \gamma^{t_{3}} W\right]
$$

where the factor $(\lambda \bar{\lambda})^{6}$ has been omitted. The index, $m^{\prime}$, appears in (88). Although we did not run a complete analysis, the result has been obtained from what we believe a few generic forms that could cover many different contributions. At some point of algebra, those apparent differences do not matter too much thanks to the anti-symmetry, (15), and various Fierz identities.

One aspect of the computation deserves a comment. In Appendix C, it is discussed for the two-loop case that the terms of the form $\left(\ldots \gamma^{p} r\right)\left(\ldots \gamma_{p} r\right)$ tend to have pairwise cancelations. We show here that for the three-loop case as well they have a stronger tendency to vanish. A given expression gets simplified by repeatedly applying various Fierz identities and the pure spinor constraints. At the final stage of the process, typically a term of the following form arrives

$$
(\bar{\lambda} \lambda)^{6}(\lambda r)\left(\lambda \gamma^{r^{\prime}} \gamma^{s} r\right)\left(W \gamma^{m^{\prime}} \gamma^{s} \gamma^{r} \gamma^{r^{\prime}} r\right)\left(r \gamma^{r} \gamma^{q^{\prime}} W\right)\left(\lambda \gamma^{q^{\prime}} W\right)(r W)
$$

When one moves $\gamma^{s}$ in $\left(W \gamma^{m^{\prime}} \gamma^{s} \gamma^{r} \gamma^{r^{\prime}} r\right)$ to the right, one generates a few terms of the form given in (103). Moving it to the far right and focusing on the two middle factors, one gets

$$
\begin{array}{r}
\left(\lambda \gamma^{r^{\prime}} \gamma^{s} r\right)\left(W \gamma^{m^{\prime}} \gamma^{r} \gamma^{r^{\prime}} \gamma^{s} r\right) \\
\sim \quad\left(\lambda \gamma^{r^{\prime}} \gamma^{0} r\right)\left(W \gamma^{m^{\prime}} \gamma^{r} \gamma^{r^{\prime}} \gamma^{0} r\right)
\end{array}
$$

where in the second line we have used a Fierz identity that is derived in Appendix C. Since there is a factor of $(\lambda r)$ in (104), one can freely shift the location of $\gamma^{r^{\prime}}$ as

$$
\sim\left(\lambda \gamma^{0} \gamma^{r^{\prime}} r\right)\left(W \gamma^{m^{\prime}} \gamma^{r} \gamma^{0} \gamma^{r^{\prime}} r\right)
$$

Application of the Fierz identity one more time brings another factor of $(\lambda r)$, hence the expression vanishes. In Appendix $\mathrm{C}$, It is shown that each contribution in the three-loop can be put into the form of $\left(\ldots \gamma^{p} r\right)\left(\ldots \gamma_{p} r\right)$. Together with the the two-loop

\footnotetext{
${ }^{16}$ The analysis still requires a large amount of algebra because each case has many sub-cases.
} 
case it may be taken as another indication that, with (66), the overall coefficient of (103) might vanish. If it is indeed zero, the role of a gauge invariant regulator will be simply to replace with by a non-zero number, as mentioned previously.

We take (103) as a three-loop analogue of (53). It is proposed based on the tendency that has been observed in all of the cases analyzed so far: the amplitude structures such as (53) and (103) reside in the individual terms that are generated by the different $d_{0}$-structures and the terms that come via an expansion of the regulator. The powerful antisymmetry made it possible to run a generic analysis because many apparently different-looking terms will correspond to different contractions that are allowed by the anti-symmetry. As for the case of $\left(d \gamma^{(3)} d\right)^{6}$, without any operator product among the non-zero modes, all of our attempts to get an analogue of (103) have led to vanishing results.

\section{Conclusion}

After reviewing the one-loop and directly computing the two-loop, we have tackled the three-loop amplitude. For the three-loop, we have used the regulator recently proposed by Aisaka and Berkovits. Based on a few indications, we have discussed the possibility that the $\mathrm{AB}$ regulator might lead to a vanishing result. Putting the issue aside, we have argued that it is possible to acquire the three-loop amplitude. The result has been presented in (103). Strictly speaking, the result is conjectural in the sense that not all the contributions are explicitly evaluated. We still believe that the result is likely to be confirmed by future analyses.

To fully verify (103), there are several things that need to be done. One obvious thing is to work out the extra contribution that was mentioned in (67). It does not pose any new difficulty or subtlety: it is just additional algebra of the same kind. (Although not recorded, a few terms were analyzed and shown to vanish. The analysis thereof is by no means extensive.) To our view, the task of the highest priority is an improved understanding of the three-loop regulator. As pointed out in the section 2 (and also in later sections), there are no contributions from any of the higher order $\frac{1}{\lambda \lambda}$-terms in $\mathcal{A}_{3 \text {-loop }}$ that come from the six $b$-insertions and have made the two-loop regulator invalid as a three-loop regulator. It would be more natural if the $\mathcal{A}_{3 \text {-loop }}$ received some contributions from those higher order terms. Checking whether the AB regulator yields a vanishing amplitude by direct computation will be an extremely tedious task. It would be far better if one could rely on some sort of sophisticated argument. Also it might be worth trying to come up with a new regulator that has this feature. A partial check can be provided by fully evaluating (103) to get a momentum space expression. One can see whether the resulting momentum space expression has some expected symmetry such as cyclicity. One may compare its field theory limit with the corresponding SYM three-loop computation.

Although the role of a new regulator (or a gauge invariant regulator, in case a new 
regulator with a different gauge fixing is not easy to come by) may be simply to change the overall numerical coefficient of (103), we cannot entirely exclude the possibility that it might introduce a new term that is different from (103). At most it will be a few more terms if any. (The contribution of (67) should also be checked against this possibility.) Checking more examples will be useful to strengthen the result, (103). Once a new regulator or a gauge invariant regulator is used, some of the contributions could come from the terms in higher powers in $\frac{1}{\lambda \lambda}$ than those in (88). A gauge invariant regulator will include more diverse structures in the pure spinor field, $\lambda$ and its conjugate. The computation using a new regulator or a gauge invariant regulator -which is one future direction- is expected to be more complex. We expect the techniques and procedures presented in this work to be useful in future computations. Progress on these issues will be reported.

\section{Acknowledgements}

I thank Y. Aisaka, N. Berkovits, C. Mafra and P. van Hove for their communications throughout the project. I especially thank H. Kawai for arranging my trips to Kyoto University, Okayama Institute of Quantum Physics and RIKEN. I am also grateful for their hospitality during my visits. I acknowledge the international visitor's program of KEK and thank Y. Kitazawa for his hospitality during my visit. Part of the work was carried out during my stay at Asia Pacific Center for Theoretical Physics and I thank Y. Kim for his hospitality. 


\section{Appendix A: Notations}

Our index conventions are

$$
\begin{aligned}
& \mu, a, m \text { etc }: 10 \mathrm{D} \text { vector indices } \\
& \alpha, \beta \text { etc }: 10 \mathrm{D} \text { spinor indices }
\end{aligned}
$$

As for the gamma matrices, we distinguish the 32 by 32 gamma matrices, $\Gamma^{m}$, from the 16 by 16 ones, $\gamma^{m}$,

$$
\Gamma^{m}=\left(\begin{array}{cc}
0 & \left(\gamma^{m}\right)_{\alpha \beta} \\
\left(\gamma^{m}\right)^{\alpha \beta} & 0
\end{array}\right),
$$

For example, $\Gamma^{m n}$ takes the form of

$$
\Gamma^{m n}=\left(\begin{array}{cc}
\left(\gamma^{m n}\right)_{\alpha}^{\beta} & 0 \\
0 & \left(\gamma^{m n}\right)^{\alpha}{ }_{\beta}
\end{array}\right),
$$

in this representation. When taking the transpose in the 32-component it is convenient to use an explicit representation of $\Gamma^{0}$. Without loss of generality one can choose

$$
\Gamma^{0}=\left(\begin{array}{cc}
0 & 1 \\
-1 & 0
\end{array}\right)
$$

When applying the 32-component Fierzing, we embed the 16-component spinors in 32-component ones

$$
\begin{aligned}
\lambda_{u} & =\left(\begin{array}{c}
\lambda^{\alpha} \\
0
\end{array}\right), \lambda_{d}=\left(\begin{array}{c}
0 \\
\lambda^{\alpha}
\end{array}\right) \\
r_{u} & =\left(\begin{array}{c}
r_{\alpha} \\
0
\end{array}\right), r_{d}=\left(\begin{array}{c}
0 \\
r_{\alpha}
\end{array}\right) \\
W_{u} & =\left(\begin{array}{c}
W^{\alpha} \\
0
\end{array}\right), \quad W_{d}=\left(\begin{array}{c}
0 \\
W^{\alpha}
\end{array}\right)
\end{aligned}
$$

and similarly for other fields. The 16 by 16 gamma matrices satisfy

$$
\eta_{m n} \gamma_{\alpha(\beta}^{m} \gamma_{\gamma \delta)}^{n}=0
$$

We often write $d^{I}$ instead of $d_{0}^{I}$ for the simplicity of notation. In the abuse of the notation, we often use

$$
\begin{aligned}
& d^{I=1} \equiv d \\
& d^{I=2} \equiv d^{\prime} \\
& d^{I=3} \equiv d^{\prime \prime}
\end{aligned}
$$


A dotted equality, "三”, represents an equality, "=", up to an overall numerical factor,

$$
\text { " } \doteq \text { denotes " = "up to an overall numerical factor }
$$

In some equations, the symbol, " ", is used instead. The symbol, $\oplus$, indicates

$\oplus$ : relative numerical coefficients are not being recorded precisely

For example, $A \oplus B$ means $A+B$ with the relative numerical coefficients between $A$ and $B$ inaccurate.

\section{Appendix B: identities}

For 32 by 32 gamma matrices, one can show that

$$
\begin{aligned}
& \Gamma^{a} \Gamma_{a}=10, \quad \Gamma^{a} \Gamma^{\mu} \Gamma_{a}=-8 \Gamma^{\mu}, \quad \Gamma^{a} \Gamma^{\mu \nu} \Gamma_{a}=6 \Gamma^{\mu \nu} \\
& \Gamma^{a} \Gamma^{\mu \nu \rho} \Gamma_{a}=-4 \Gamma^{\mu \nu \rho}, \quad \Gamma^{a} \Gamma^{\mu \nu \rho \sigma} \Gamma_{a}=2 \Gamma^{\mu \nu \rho \sigma}, \quad \Gamma^{a} \Gamma^{\mu \nu \rho \sigma \delta} \Gamma_{a}=0 \\
& \Gamma^{a} \Gamma^{\mu \nu \rho \sigma \delta \kappa} \Gamma_{a}=-2 \Gamma^{\mu \nu \rho \delta \kappa}, \quad \Gamma^{a} \Gamma^{\mu \nu \rho \sigma \delta \kappa \zeta} \Gamma_{a}=4 \Gamma^{\mu \nu \rho \sigma \delta \kappa \zeta} \\
& \Gamma^{a b} \Gamma_{a b}=-90, \quad \Gamma^{a b} \Gamma^{\mu} \Gamma_{a b}=-54 \Gamma^{\mu}, \quad \Gamma^{a b} \Gamma^{\mu \nu} \Gamma_{a b}=-26 \Gamma^{\mu \nu} \\
& \Gamma^{a b} \Gamma^{\mu \nu \rho} \Gamma_{a b}=-6 \Gamma^{\mu \nu \rho}, \quad \Gamma^{a b} \Gamma^{\mu \nu \rho \sigma} \Gamma_{a b}=6 \Gamma^{\mu \nu \rho \sigma}, \quad \Gamma^{a b} \Gamma^{\mu \nu \rho \sigma \delta} \Gamma_{a b}=10 \Gamma^{\mu \nu \rho \sigma \delta}, \\
& \Gamma^{a b} \Gamma^{\mu \nu \rho \sigma \delta \kappa} \Gamma_{a b}=6 \Gamma^{\mu \nu \rho \sigma \delta \kappa} \\
& \Gamma^{a b c} \Gamma_{a b c}=-720, \quad \Gamma^{a b c} \Gamma^{\mu} \Gamma_{a b c}=288 \Gamma^{\mu}, \quad \Gamma^{a b c} \Gamma^{\mu \nu} \Gamma_{a b c}=-48 \Gamma^{\mu \nu} \\
& \Gamma^{a b c} \Gamma^{\mu \nu \rho} \Gamma_{a b c}=-48 \Gamma^{\mu \nu \rho}, \quad \Gamma^{a b c} \Gamma^{\mu \nu \rho \sigma} \Gamma_{a b c}=48 \Gamma^{\mu \nu \rho \sigma}, \quad \Gamma^{a b c} \Gamma^{\mu \nu \rho \sigma \delta} \Gamma_{a b c}=0 \\
& \Gamma^{a b c} \Gamma^{\mu \nu \rho \sigma \delta \kappa} \Gamma_{a b c}=-48 \Gamma^{\mu \nu \rho \sigma \delta \kappa} \\
& \Gamma^{a b c d} \Gamma_{a b c d}=5040, \quad \Gamma^{a b c d} \Gamma^{\mu} \Gamma_{a b c d}=1008 \Gamma^{\mu}, \quad \Gamma^{a b c d} \Gamma^{\mu \nu} \Gamma_{a b c d}=-336 \Gamma^{\mu \nu} \\
& \Gamma^{a b c d} \Gamma^{\mu \nu \rho} \Gamma_{a b c d}=-336 \Gamma^{\mu \nu \rho}, \quad \Gamma^{a b c d} \Gamma^{\mu \nu \rho \sigma} \Gamma_{a b c d}=48 \Gamma^{\mu \nu \rho \sigma} \\
& \Gamma^{a b c d} \Gamma^{\mu \nu \rho \sigma \delta} \Gamma_{a b c d}=240 \Gamma^{\mu \nu \rho \sigma \delta}, \quad \Gamma^{a b c d} \Gamma^{\mu \nu \rho \sigma \delta \kappa} \Gamma_{a b c d}=48 \Gamma^{\mu \nu \rho \sigma \delta \kappa} \\
& \Gamma^{a b c d e} \Gamma_{a b c d e}=6 \cdot 5040, \quad \Gamma^{a b c d e} \Gamma^{\mu} \Gamma_{a b c d e}=0, \quad \Gamma^{a b c d e} \Gamma^{\mu \nu} \Gamma_{a b c d e}=-3360 \Gamma^{\mu \nu}, \\
& \Gamma^{a b c d e} \Gamma^{\mu \nu \rho} \Gamma_{a b c d e}=0, \quad \Gamma^{a b c d e} \Gamma^{\mu \nu \rho \sigma} \Gamma_{a b c d e}=1440 \Gamma^{\mu \nu \rho \sigma} \\
& \Gamma^{a b c d e} \Gamma^{\mu \nu \rho \sigma \delta} \Gamma_{a b c d e}=0
\end{aligned}
$$

They also satisfy ${ }^{17}$

$$
\begin{aligned}
& {\left[\Gamma_{m}, \Gamma^{r}\right]=2 \Gamma_{m}^{r} \quad, \quad\left\{\Gamma_{m}, \Gamma^{r}\right\}=2 \delta_{m}^{r}} \\
& \left\{\Gamma_{m n}, \Gamma^{r}\right\}=2 \Gamma_{m n}{ }^{r} \quad, \quad\left[\Gamma_{m n}, \Gamma^{r}\right]=-4 \delta_{[m}^{r} \Gamma_{n]} \\
& {\left[\Gamma_{m n p}, \Gamma^{r}\right]=2 \Gamma_{m n p}{ }^{r} \quad, \quad\left\{\Gamma_{m n p}, \Gamma^{r}\right\}=6 \delta_{[m}^{r} \Gamma_{n p]}} \\
& \left\{\Gamma_{m n p q}, \Gamma^{r}\right\}=2 \Gamma_{m n p q}{ }^{r} \quad, \quad\left[\Gamma_{m n p q}, \Gamma^{r}\right]=-8 \delta_{[m}^{r} \Gamma_{n p q]} \\
& {\left[\Gamma_{m n p q k}, \Gamma^{r}\right]=2 \Gamma_{m n p q k}{ }^{r} \quad, \quad\left\{\Gamma_{m n p q k}, \Gamma^{r}\right\}=10 \delta_{[m}^{r} \Gamma_{n p q k]}}
\end{aligned}
$$

\footnotetext{
${ }^{17}$ Many of them can be found in [37] where a few of the coefficients are erroneous.
} 


$$
\begin{aligned}
& \left.\left\{\Gamma_{m n}, \Gamma^{r s}\right\}=2 \Gamma_{m n}^{r s}-4 \delta_{[m n]}^{r s} \quad, \quad\left[\Gamma_{m n}, \Gamma^{r s}\right]=-8 \delta_{[m}^{[r} \Gamma_{n]} s\right] \\
& \left\{\Gamma_{m n p}, \Gamma^{r s}\right\}=2 \Gamma_{m n p}^{r s}-12 \delta_{[m n}^{r s} \Gamma_{p]} \quad, \quad\left[\Gamma_{m n p}, \Gamma^{r s}\right]=12 \delta_{[m}^{[r} \Gamma_{n p]}^{s]} \\
& \left.\left\{\Gamma_{m n p q}, \Gamma^{r s}\right\}=2 \Gamma_{m n p q}^{r s}-24 \delta_{[m n}^{r s} \Gamma_{p q]} \quad, \quad\left[\Gamma_{m n p q}, \Gamma^{r s}\right]=-16 \delta_{[m}^{[r} \Gamma_{n p q]} s\right] \\
& \left\{\Gamma_{m n p q k}, \Gamma^{r s}\right\}=2 \Gamma_{m n p q k}^{r s}-40 \delta_{[m n}^{r s} \Gamma_{p q k]} \quad, \quad\left[\Gamma_{m n p q k}, \Gamma^{r s}\right]=20 \delta_{[m}^{[r} \Gamma_{n p q k]}^{s]} \\
& {\left[\Gamma_{m n p}, \Gamma^{r s t}\right]=2 \Gamma_{m n p}^{r s t}-36 \delta_{[m n}^{[r s} \Gamma_{p]}^{t]} \quad\left\{\Gamma_{m n p}, \Gamma^{r s t}\right\}=18 \delta_{[m}^{[r} \Gamma_{n p}{ }^{r s]}-12 \delta_{[m n p]}^{r s t}} \\
& \left\{\Gamma_{m n p q}, \Gamma^{r s t}\right\}=2 \Gamma_{m n p q}^{r s t}-72 \delta_{[m n}^{r s} \Gamma_{p q]}{ }^{t]} \\
& {\left[\Gamma_{m n p q}, \Gamma^{r s t}\right]=-24 \delta_{[m}^{[r} \Gamma_{n p q]}^{s t]}+48 \delta_{[m n p}^{r s t} \Gamma_{q]}} \\
& \left\{\Gamma_{m n p q}, \Gamma^{r s t u}\right\}=2 \Gamma_{m n p q}^{r s t u}-144 \delta_{[m n}^{r s} \Gamma_{p q]}{ }^{t u]}+48 \delta_{[m n p q]}^{r s t u}, \\
& \left.\left[\Gamma_{m n p q}, \Gamma^{r s t u}\right]=-32 \delta_{[m}^{[r} \Gamma_{n p q} s t u\right]-64 \delta_{[m n p}^{[r s t} \Gamma_{q}^{u]} \\
& \left\{\Gamma_{m n p q k}, \Gamma^{r s t}\right\}=30 \delta_{[m}^{[r} \Gamma_{n p q k}^{s t]}-120 \delta_{[m n p}^{[r s t} \Gamma_{q k]} \\
& \left.\left[\Gamma_{m n p q k}, \Gamma^{r s t}\right]=2 \Gamma_{m n p q k}^{r s t}-120 \delta_{[m n}^{[r s} \Gamma_{p q k]}\right]^{t]} \\
& \left\{\Gamma_{m n p q k}, \Gamma^{r s t u}\right\}=2 \Gamma_{m n p q k}^{r s t u}-240 \delta_{[m n}^{[r s} \Gamma_{p q k}^{t u]}+240 \delta_{[m n p q}^{[r s t u} \Gamma_{k]} \\
& {\left[\Gamma_{m n p q k}, \Gamma^{r s t u}\right]=40 \delta_{[m}^{[r} \Gamma_{n p q k}^{s t u]}-480 \delta_{[m n p}^{[r s t} \Gamma_{q k]}^{u]}}
\end{aligned}
$$

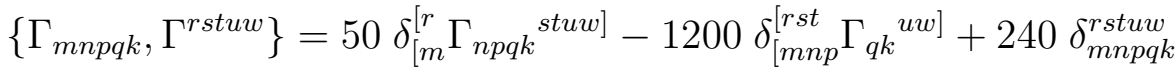

$$
\begin{aligned}
& \left.\left[\Gamma_{m n p q k}, \Gamma^{r s t u w}\right]=2 \Gamma_{m n p q k}^{r s t u w}-400 \delta_{[m n}^{[r s} \Gamma_{p q k}{ }^{t u w]}+1200 \delta_{[m n p q}^{[r s t u} \Gamma_{k]} w\right] \\
& \left\{\Gamma_{m n p q k l}, \Gamma^{r}\right\}=2 \Gamma_{m n p q k l}{ }^{r}, \quad\left[\Gamma_{m n p q k l}, \Gamma^{r}\right]=-12 \delta_{[m}^{r} \Gamma_{n p q k l]} \\
& \left.\left\{\Gamma_{m n p q k l}, \Gamma^{r s}\right\}=2 \Gamma_{m n p q k l}^{r s}-60 \delta_{[m n}^{[r s} \Gamma_{p q k l]}, \quad\left[\Gamma_{m n p q k l}, \Gamma^{r s}\right]=-24 \delta_{[m}^{[r} \Gamma_{n p q k l]} s\right] \\
& \left\{\Gamma_{m n p q k l}, \Gamma^{r s t}\right\}=2 \Gamma_{m n p q k l}^{r s t}-180 \delta_{[m n}^{[r s} \Gamma_{p q k l]}^{t]} \\
& {\left[\Gamma_{m n p q k l}, \Gamma^{r s t}\right]=-36 \delta_{[m}^{[r} \Gamma_{n p q k l}^{s t]}+240 \delta_{[m n p}^{r s t} \Gamma_{q k l]}} \\
& \left\{\Gamma_{m n p q k l}, \Gamma^{r s t u}\right\}=2 \Gamma_{m n p q k l}^{r s t u}-360 \delta_{[m n}^{[r s} \Gamma_{p q k l}^{t u]}+720 \delta_{[m n p q}^{r s t u} \Gamma_{k l]} \\
& {\left[\Gamma_{m n p q k l}, \Gamma^{r s t u}\right]=-48 \delta_{[m}^{[r} \Gamma_{n p q k l}{ }^{s t u]}+960 \delta_{[m n p}^{[r s t} \Gamma_{q k l]}^{u]}} \\
& \left.\left\{\Gamma_{m n p q k l}, \Gamma^{r s t u w}\right\}=-600 \delta_{[m n}^{[r s} \Gamma_{p q k l}{ }^{t u w]}+3600 \delta_{[m n p q}^{[r s t u} \Gamma_{k l]} w\right] \\
& \left.\left[\Gamma_{m n p q k l}, \Gamma^{r s t u w}\right]=-60 \delta_{[m}^{[r} \Gamma_{n p q k l}{ }^{s t u w]}+2400 \delta_{[m n p}^{[r s t} \Gamma_{q k l}^{u w}\right]-1440 \delta_{[m n p q k}^{r s t u w} \Gamma_{l]}
\end{aligned}
$$

In some manipulations, we used

$$
\begin{aligned}
\Gamma^{\mu_{1} \ldots \mu_{4}}= & \Gamma^{\mu_{4}} \ldots \Gamma^{\mu_{1}}-\eta^{\mu_{1} \mu_{2}} \Gamma^{\mu_{4}} \Gamma^{\mu_{3}}-2 \Gamma^{\mu_{4}} \eta^{\mu_{3}\left[\mu_{2}\right.} \Gamma^{\left.\mu_{1}\right]}+3 \eta^{\mu_{4}\left[\mu_{1}\right.} \Gamma^{\left.\mu_{2} \mu_{3}\right]} \\
= & \Gamma^{\mu_{1}} \ldots \Gamma^{\mu_{4}}-\eta^{\mu_{1} \mu_{2}} \Gamma^{\mu_{3}} \Gamma^{\mu_{4}}+2 \eta^{\mu_{3}\left[\mu_{1}\right.} \Gamma^{\left.\mu_{2}\right]} \Gamma^{\mu_{4}}-3 \eta^{\mu_{4}\left[\mu_{1}\right.} \Gamma^{\left.\mu_{2} \mu_{3}\right]} \\
\Gamma^{\mu_{1} \ldots \mu_{3}}= & -\Gamma^{\mu_{3}} \Gamma^{\mu_{2}} \Gamma^{\mu_{1}}+\eta^{\mu_{1} \mu_{2}} \Gamma^{\mu_{3}}-2 \eta^{\mu_{3}\left[\mu_{1}\right.} \Gamma^{\left.\mu_{2}\right]} \\
= & \Gamma^{\mu_{1}} \Gamma^{\mu_{2}} \Gamma^{\mu_{3}}-\eta^{\mu_{1} \mu_{2}} \Gamma^{\mu_{3}}+2 \eta^{\mu_{3}\left[\mu_{1}\right.} \Gamma^{\left.\mu_{2}\right]} \\
\Gamma^{\mu_{1} \ldots \mu_{5}}= & \Gamma^{\mu_{5}} \ldots \Gamma^{\mu_{1}}-\eta^{\mu_{1} \mu_{2}} \Gamma^{\mu_{5}} \Gamma^{\mu_{4}} \Gamma^{\mu_{3}}-2 \Gamma^{\mu_{5}} \Gamma^{\mu_{4}} \eta^{\mu_{3}\left[\mu_{2}\right.} \Gamma^{\left.\mu_{1}\right]} \\
& +3 \Gamma^{\mu_{5}} \eta^{\mu_{4}\left[\mu_{1}\right.} \Gamma^{\left.\mu_{2} \mu_{3}\right]}-4 \eta^{\mu_{5}\left[\mu_{1}\right.} \Gamma^{\left.\mu_{2} \mu_{3} \mu_{4}\right]}
\end{aligned}
$$


which are straightforward to verify. For commuting spinors, the Fierz rearrangement is

$$
\left(\chi_{1}^{\dagger} M \chi_{2}\right)\left(\chi_{3}^{\dagger} N \chi_{4}\right)=\sum_{I}\left(\chi_{1}^{\dagger} O^{I} \chi_{4}\right)\left(\chi_{3}^{\dagger} N O^{I} M \chi_{2}\right) \frac{1}{\operatorname{tr}\left(O_{I}^{2}\right)}
$$

For anti-commuting spinors, there will be an overall minus sign. By choosing $M, N$ and $\chi$ 's appropriately one can derive the 16component Fierz relations. For example, with $M=\Gamma^{m}, N=\Gamma^{m}, \chi_{1}=\left(1_{\alpha}, 0\right), \chi_{2}=\left(0,1_{\beta}\right), \chi_{3}=\left(1_{\sigma}, 0\right), \chi_{4}=\left(0,1_{\delta}\right)$, one gets

$$
\gamma_{\alpha \beta}^{m} \gamma_{\delta \sigma}^{m}=-\frac{1}{2} \gamma_{\alpha \delta}^{m} \gamma_{\beta \sigma}^{m}+\frac{1}{24} \gamma_{\alpha \delta}^{m n p} \gamma_{\sigma \beta}^{m n p}
$$

Taking $M=\Gamma^{m n p q}, N=\Gamma^{m n p q}$, one gets

$$
\left(\gamma_{m n p q}\right)_{\alpha}{ }^{\beta}\left(\gamma_{m n p q}\right)_{\sigma}{ }^{\delta}=315 \delta_{\alpha}^{\delta} \delta_{\sigma}^{\beta}+\frac{21}{2}\left(\gamma^{m n}\right)_{\alpha}{ }^{\delta}\left(\gamma^{m n}\right)_{\sigma}{ }^{\beta}+\frac{1}{8}\left(\gamma^{m n p q}\right)_{\alpha}{ }^{\delta}\left(\gamma^{m n p q}\right)_{\sigma}{ }^{\beta}
$$

Taking $M=\Gamma^{m n p}, N=\Gamma^{m n p}$ with the same choice of $\chi$ 's gives, ${ }^{18}$

$$
\gamma_{\alpha \beta}^{a b c} \gamma_{\delta \sigma}^{a b c}=18 \gamma_{\alpha \delta}^{m} \gamma_{\sigma \beta}^{m}+\frac{1}{2} \gamma_{\alpha \delta}^{m n p} \gamma_{\sigma \beta}^{m n p}
$$

Taking either of the equation above and anti-symmetrizing $(\beta \sigma \delta)$, one can easily show

$$
\gamma_{\alpha(\beta}^{m} \gamma_{\delta \sigma)}^{m}=0
$$

Another useful identity given in [35] is

$$
\left(\gamma^{\mu \nu}\right)_{\alpha}{ }^{\delta}\left(\gamma^{\mu \nu}\right)_{\beta}^{\sigma}=-8 \delta_{\alpha}^{\sigma} \delta_{\beta}^{\delta}+4\left(\gamma^{\mu}\right)_{\alpha \beta}\left(\gamma^{\mu}\right)^{\delta \sigma}-2 \delta_{\alpha}^{\delta} \delta_{\beta}^{\sigma}
$$

For $\operatorname{tr}\left(O_{I}^{2}\right)$, note that one can make the following replacements,

$$
\begin{aligned}
& \operatorname{tr}(1) \rightarrow 32, \quad \operatorname{tr}\left(\left(\Gamma^{(2)}\right)^{2}\right) \rightarrow-64, \quad \operatorname{tr}\left(\left(\Gamma^{(4)}\right)^{2}\right) \rightarrow 768, \quad \operatorname{tr}\left(\left(\Gamma^{(6)}\right)^{2}\right) \rightarrow-6 ! \cdot 32 \\
& \operatorname{tr}\left(\left(\Gamma^{(8)}\right)^{2}\right) \rightarrow 8 ! \cdot 32, \quad \operatorname{tr}\left(\left(\Gamma^{(10)}\right)^{2}\right) \rightarrow-10 ! \cdot 32 \\
& \operatorname{tr}\left(\Gamma^{(1)}\right) \rightarrow 32, \quad \operatorname{tr}\left(\left(\Gamma^{(3)}\right)^{2}\right) \rightarrow-3 ! \cdot 32, \quad \operatorname{tr}\left(\left(\Gamma^{(5)}\right)^{2}\right) \rightarrow 5 ! \cdot 32, \quad \operatorname{tr}\left(\left(\Gamma^{(7)}\right)^{2}\right) \rightarrow-7 ! \cdot 32 \\
& \operatorname{tr}\left(\left(\Gamma^{(9)}\right)^{2}\right) \rightarrow 9 ! \cdot 32
\end{aligned}
$$

The following relations were used when a transpose of a 32 by 32 gamma matrix was taken,

$$
\begin{aligned}
\left(\Gamma^{\mu}\right)^{T} & =\Gamma^{0} \Gamma^{\mu} \Gamma^{0} \\
\left(\Gamma^{\mu \nu}\right)^{T} & =\Gamma^{0} \Gamma^{\mu \nu} \Gamma^{0} \\
\left(\Gamma^{\mu \nu \rho}\right)^{T} & =-\Gamma^{0} \Gamma^{\mu \nu \rho} \Gamma^{0} \\
\left(\Gamma^{\mu \nu \rho \sigma}\right)^{T} & =-\Gamma^{0} \Gamma^{\mu \nu \rho \sigma} \Gamma^{0} \\
\left(\Gamma^{\mu_{1} \ldots \mu_{5}}\right)^{T} & =\Gamma^{0} \Gamma^{\mu_{1} \ldots \mu_{5}} \Gamma^{0}
\end{aligned}
$$

The fact that $\lambda_{u} \Gamma^{\mu \nu \rho} \lambda_{d}=0$ can be seen, e.g., by using the transpose relations given above.

\footnotetext{
${ }^{18}$ Compared with (62) of [29], there is overall minus sign difference.
} 


\section{Appendix C: Aisaka-Berkovits regulator, cont'd}

After the $[d s]$ - and $[d d]$ - integrations, a given contribution becomes an equation where the variables, ( $\lambda$ 's, $\bar{\lambda}$ 's, $r$ 's, $\gamma^{m_{1} \ldots m_{j}}$ 's) get contracted among themselves and/or with the SYM fields. It gets simplified by applying the Fierz identities and the pure spinor constraints. While doing so, one encounters the following form of factors,

$$
\left(\ldots \gamma^{p} \gamma^{m} r\right)\left(\ldots \gamma_{p} r\right)
$$

We write this as $\left(\chi \gamma^{p} \gamma^{m} r\right)\left(\xi \gamma_{p} r\right)$ which in turn can be rewritten,

$$
\left(\chi \gamma^{p} \gamma^{m} r\right)\left(\xi \gamma_{p} r\right)=2(\chi r)\left(\xi \gamma^{m} r\right)-\left(\chi^{\prime} \gamma^{p} r\right)\left(\xi \gamma_{p} r\right)
$$

where $\chi^{\prime} \equiv \chi \gamma^{m}$. Let us focus on the second term. The Fierz identity, $r_{\alpha} r_{\beta} \sim$ $\left(\gamma^{\mu \nu \rho}\right)_{\alpha \beta}\left(r \gamma_{\mu \nu \rho} r\right)$, yields

$$
\left(\chi \gamma^{p} r\right)\left(\xi \gamma_{p} r\right) \sim\left(\chi \gamma^{\mu \nu \rho} \xi\right)\left(r \gamma_{\mu \nu \rho} r\right),
$$

The prime on $\chi^{\prime}$ has been dropped. It does not admit the inverse Fierzing right away because of the changed index structure. One can "force" the inverse Fierzing by temporarily going to the 32-component notation with the first factor,

$$
\begin{aligned}
& \sim\left(\chi_{d} \Gamma^{\mu \nu \rho} \xi_{u}\right)\left(r \gamma_{\mu \nu \rho} r\right) \\
& \sim\left(\xi_{u}\left(\Gamma^{\mu \nu \rho}\right)^{T} \chi_{d}\right)\left(r \gamma_{\mu \nu \rho} r\right) \\
& \sim\left(\xi_{u} \Gamma^{0} \Gamma^{\mu \nu \rho} \Gamma^{0} \chi_{d}\right)\left(r \gamma_{\mu \nu \rho} r\right)
\end{aligned}
$$

where in the third line one of the gamma matrix identity has been used. Moving back to the 16-component notation, one gets

$$
\sim\left(\xi \gamma^{0} \gamma^{\mu \nu \rho} \gamma^{0} \chi\right)\left(r \gamma_{\mu \nu \rho} r\right)
$$

The matrix, $\gamma^{0}$, is $\delta^{\alpha \beta}$ up to a sign. Its role is to bring back the index structure so now the inverse Fierzing can be carried out,

$$
\left(\chi \gamma^{p} r\right)\left(\xi \gamma_{p} r\right) \sim\left(\chi \gamma^{0} r\right)\left(\xi \gamma^{0} r\right)
$$

Note that by forcing the inverse Fierzing, extra $\gamma^{0}$ 's have been introduced. As a matter of fact, what we amount to show below is that all the structures that do not contain a single factor of $\left(\chi^{\prime} \gamma^{p} r\right)\left(\xi \gamma_{p} r\right)$ drop out due to various constraints and identities. Eq.(C.6) has an interesting implication. We illustrate it with a specific example. In the two-loop analysis of the $\left(d \gamma^{(3)} d\right)^{5}$-term, one typically encounters a term such as

$$
(r \lambda)\left(\lambda \gamma^{r} W_{(1)}\right)\left(\lambda \gamma^{s^{\prime}} W_{(3)}\right)\left(r \gamma^{s^{\prime}} \gamma^{q^{\prime}} W_{(4)}\right)\left(r \gamma^{q^{\prime}} \gamma^{r} W_{(2)}\right)
$$

at some stage of the manipulation. Writing

$$
\left(r \gamma^{s^{\prime}} \gamma^{q^{\prime}} W_{(4)}\right)=-\left(r \gamma^{q^{\prime}} \gamma^{s^{\prime}} W_{(4)}\right)+2\left(r W_{(4)}\right)
$$


we focus on the first term since the second term yields the desired two-loop expression, (53). With the other factors, the first term gives

$$
(r \lambda)\left(\lambda \gamma^{r} W_{(1)}\right)\left(\lambda \gamma^{s^{\prime}} W_{(3)}\right)\left(r \gamma^{q^{\prime}} \gamma^{s^{\prime}} W_{(4)}\right)\left(r \gamma^{q^{\prime}} \gamma^{r} W_{(2)}\right)
$$

Substituting (C.6), one gets

$$
(r \lambda)\left(\lambda \gamma^{r} W_{(1)}\right)\left(\lambda \gamma^{s^{\prime}} W_{(3)}\right)\left(r \gamma^{0} \gamma^{s^{\prime}} W_{(4)}\right)\left(r \gamma^{0} \gamma^{r} W_{(2)}\right)
$$

which looks more "uniform" compared with (53). This term gets canceled by a term that is present due to the permutation of the external states,

$$
(r \lambda)\left(\lambda \gamma^{r} W_{(3)}\right)\left(\lambda \gamma^{s^{\prime}} W_{(1)}\right)\left(r \gamma^{0} \gamma^{s^{\prime}} W_{(2)}\right)\left(r \gamma^{0} \gamma^{r} W_{(4)}\right)
$$

as one can easily check by shifting around $W^{\prime}$ 's and relabeling the indices, $\left(r, s^{\prime}\right)$.

We come to the task of showing that the entire three-loop contributions with the regulator (66) can be put into the forms that contain at least one factor of $\left(\ldots \gamma^{p} r\right)\left(\ldots \gamma^{p} r\right)$. Mostly the analysis of $\left(d \gamma^{(3)} d\right)^{5}$-case is presented. The analysis of $\left(d \gamma^{(3)} d\right)^{6}$-case is simpler. We have conducted complete analysis examining all the sub-cases for the following cases,

$$
\begin{aligned}
\left(n_{d}, n_{d^{\prime}}, n_{d^{\prime \prime}}\right)=\quad & (11,4,0),(11,3,1),(11,2,2),(10,5,0), \\
& (10,4,1),(10,3,2),(7,5,3),(6,5,4)
\end{aligned}
$$

and run partial checks on,

$$
\left(n_{d}, n_{d^{\prime}}, n_{d^{\prime \prime}}\right)=(8,4,3),(7,5,3)
$$

We illustrate with $\left(n_{d}, n_{d^{\prime}}, n_{d^{\prime \prime}}\right)=(11,2,2)$. In appropriate places below, the word "vanish" means "vanish up to the terms that contain $\left(\ldots \gamma^{p} r\right)\left(\ldots \gamma_{p} r\right)$ ",

$$
\text { "vanish" = "vanish up to the terms that contain }\left(\ldots \gamma^{p} r\right)\left(\ldots \gamma_{p} r\right) "
$$

Also a "vanishing expression" means it is the form of $\left(\ldots \gamma^{p} r\right)\left(\ldots \gamma_{p} r\right)$. Frequent uses will be made of the identity, (39). Because the analyses of the two terms in (39) go similarly we present the contributions from the second term,

$$
\left(\bar{\lambda}_{d} \Gamma^{m n p} r_{u}\right)\left(\lambda_{u} \Gamma^{r} \Gamma_{m n p} \Gamma^{s} \lambda_{d}\right) \Rightarrow(\bar{\lambda} \lambda)\left(\lambda_{u} \Gamma^{r} \Gamma^{s} r_{u}\right)
$$

After the $g$ - and $d$ - integrations of the first term in (88), one gets

$$
\begin{aligned}
& \begin{array}{cccccc}
\rho_{1} \ldots \rho_{m-6} \rho_{m-5} \ldots \rho_{m} & \rho_{1}^{\prime} \ldots \rho_{n-3}^{\prime} \rho_{n-2}^{\prime} \rho_{n-1}^{\prime} \rho_{n}^{\prime} & \rho_{1}^{\prime \prime} \ldots \ldots \rho_{l}^{\prime \prime} \\
\sigma_{1} \ldots \sigma_{m-6} & \sigma_{1}^{\prime} \ldots \ldots \sigma_{6}^{\prime} & \sigma_{7}^{\prime} \ldots \sigma_{n+3}^{\prime} & \sigma_{1}^{\prime \prime} & \sigma_{2}^{\prime \prime} & \sigma_{3}^{\prime \prime} \ldots \sigma_{4}^{\prime \prime} \ldots \ldots \sigma_{l+3}^{\prime \prime}
\end{array} \\
& \left(\epsilon_{\rho_{m+1} \ldots \rho_{11} \sigma_{1} \ldots \sigma_{m-6} \kappa_{1} \ldots \kappa_{11}}\right)\left(\epsilon_{\rho_{n+1}^{\prime} \ldots \rho_{11}^{\prime} \sigma_{1}^{\prime} \ldots \sigma_{n+3}^{\prime} \kappa_{1}^{\prime} \kappa_{2}^{\prime}}\right)\left(\epsilon_{\rho_{l+1}^{\prime \prime} \ldots \rho_{11}^{\prime \prime} \sigma_{1}^{\prime \prime} \ldots \sigma_{l+3}^{\prime \prime} \kappa_{1}^{\prime \prime} \kappa_{2}^{\prime \prime}}\right)
\end{aligned}
$$

Using the Kronecker-delta in front, one gets

$$
\left(\epsilon_{\rho_{m+1} \ldots \rho_{11} \rho_{1} \ldots \rho_{m-6} \kappa_{1} \ldots \kappa_{11}}\right)\left(\epsilon_{\rho_{n+1}^{\prime} \ldots \rho_{11}^{\prime} \rho_{m-5} \ldots \rho_{m} \rho_{1}^{\prime} \ldots \rho_{n-3}^{\prime} \kappa_{1}^{\prime} \kappa_{2}^{\prime}}\right)\left(\epsilon_{\rho_{l+1}^{\prime \prime} \ldots \rho_{11}^{\prime \prime} \rho_{n-2}^{\prime} \ldots \rho_{n}^{\prime} \rho_{1}^{\prime \prime} \ldots \rho_{l}^{\prime \prime} \kappa_{1}^{\prime \prime} \kappa_{2}^{\prime \prime}}\right)(
$$


It can be contracted with other $\epsilon$ 's that come from the $[d s]$ - integration measure to yield

$$
\delta_{\kappa_{1} \ldots \ldots \ldots \ldots \kappa_{11}}^{\alpha_{1} \ldots \alpha_{5} \rho_{m-5} \ldots \rho_{m}} \quad \delta_{\kappa_{1}^{\prime} \kappa_{2}^{\prime} \rho_{m-5} \ldots \rho_{m}}^{\alpha_{1}^{\prime} \ldots \alpha_{n}^{\prime} \rho_{n-2}^{\prime} \ldots \rho_{n}^{\prime}} \quad \delta_{\kappa_{1}^{\prime \prime} \kappa_{2}^{\prime \prime} \rho_{n-2}^{\prime} \ldots \rho_{n}^{\prime}}^{\alpha^{\prime \prime} \ldots \alpha_{5}^{\prime \prime}}
$$

Fop this case not to vanish trivially, five $d_{0}$ 's out of eleven must be assigned to

$$
\left(\bar{\lambda} \gamma_{m} d\right)\left[d W\left(z_{1}\right)\right]\left[d W\left(z_{2}\right)\right]\left[d W\left(z_{3}\right)\right]\left[d W\left(z_{4}\right)\right]
$$

Otherwise two factors of a diagonal term, $\left(d^{I} \gamma^{(3)} d^{I}\right)\left(d^{I} \gamma^{(3)} d^{I}\right)$, (no sum) will be present and the expression vanishes. The factor, $\left(d \gamma_{m n p} d\right)^{5}$, in (92) takes the form of

$$
\left(\gamma_{m_{1} n_{1} p_{1}}\right)^{\kappa_{1} \kappa_{2}}\left(\gamma_{m_{2} n_{2} p_{2}}\right)^{\kappa_{3} \kappa_{1}^{\prime}}\left(\gamma_{m_{3} n_{3} p_{3}}\right)^{\kappa_{4} \kappa_{2}^{\prime}}\left(\gamma_{m_{4} n_{4} p_{4}}\right)^{\kappa_{5} \kappa_{1}^{\prime \prime}}\left(\gamma_{m_{5} n_{5} p_{5}}\right)^{\kappa_{6} \kappa_{2}^{\prime \prime}}
$$

We execute the third bundle of Kronecker-deltas in (C.18) with the following choice,

$$
\alpha_{1}^{\prime \prime}=\rho_{n-2}^{\prime}, \quad \alpha_{2}^{\prime \prime}=\rho_{n-1}^{\prime}, \quad \alpha_{3}^{\prime \prime}=\rho_{n}^{\prime}, \quad \alpha_{4}^{\prime \prime}=\kappa_{1}^{\prime \prime}, \quad \alpha_{5}^{\prime \prime}=\kappa_{2}^{\prime \prime}
$$

They lead to

$$
\begin{aligned}
& \left(\gamma_{m_{1} n_{1} p_{1}}\right)^{\kappa_{1} \kappa_{2}}\left(\gamma_{m_{2} n_{2} p_{2}}\right)^{\kappa_{3} \kappa_{1}^{\prime}}\left(\gamma_{m_{3} n_{3} p_{3}}\right)^{\kappa_{4} \kappa_{2}^{\prime}}\left(\gamma_{m_{4} n_{4} p_{4}} \gamma^{\gamma^{\prime \prime} s^{\prime \prime} q^{\prime \prime}} \gamma_{m_{5} n_{5} p_{5}}\right)^{\kappa_{5} \kappa_{6}} \\
& \delta_{\kappa_{1} \ldots \ldots \ldots \ldots \kappa_{11} \ldots \ldots \alpha_{1}}^{\alpha_{1} \ldots \alpha_{5} \rho_{m-5} \ldots \rho_{m}} \quad \delta_{\kappa_{1}^{\prime} \kappa_{2}^{\prime} \rho_{m-5}^{\prime} \ldots \rho_{m}}^{\alpha_{1}^{\prime} \alpha_{1}^{\prime \prime} \ldots \alpha_{3}^{\prime \prime}}
\end{aligned}
$$

The contractions dictated by $\delta_{\kappa_{1} \ldots \alpha_{5} \rho_{m-5} \ldots \rho_{m}}^{\alpha_{1} \ldots \ldots \kappa_{11}}$ can be classified into the following cases. (i) When $\left(\alpha_{1}, \ldots, \alpha_{5}\right) \in\left(\kappa_{1}, \ldots, \kappa_{6}\right)$, one gets, e.g., $\left(\lambda \gamma^{r} \gamma^{m_{2} n_{2} p_{2}} \gamma^{\square} \lambda\right) \operatorname{Tr}\left(\gamma^{r s q} \gamma^{m_{1} n_{1} p_{1}}\right)$ where $\square$ denotes one of the indices of $\left(r^{\prime}, s^{\prime}, q^{\prime}, r^{\prime \prime}, s^{\prime \prime}, q^{\prime \prime}\right)$. With other factors, it leads to a vanishing expression.

(ii) When four of $\left(\alpha_{1}, \ldots, \alpha_{5}\right)$ contracts among $\left(\kappa_{1}, \ldots, \kappa_{6}\right)$, one can choose the contractions to generate a factor, either of the previous type or of the form

$$
\left(\lambda \gamma^{r} \gamma^{m_{1} n_{1} p_{1}} \gamma^{s} \lambda\right) \operatorname{Tr}\left(\gamma^{r s q} \gamma^{m_{4} n_{4} p_{4}} \gamma^{r^{\prime \prime} s^{\prime \prime} q^{\prime \prime}} \gamma^{m_{5} n_{5} p_{5}}\right)
$$

which vanishes.

(iii)When $\left(\alpha_{1}, \ldots, \alpha_{5}\right)$ contracts with three out of $\left(\kappa_{1}, \ldots, \kappa_{6}\right)$ and two $\left(\kappa_{7}, \ldots, \kappa_{11}\right)$, if the three $\kappa$ 's are $\left(\kappa_{1}, \kappa_{2}, \kappa_{3}\right)$, one gets $\left(\lambda \gamma^{r} \gamma^{m_{2} n_{2} p_{2}} \gamma^{\square} \lambda\right) \operatorname{Tr}\left(\gamma^{r s q} \gamma^{m_{1} n_{1} p_{1}}\right)$. If they are $\left(\kappa_{1}, \kappa_{2}\right)$ and $\kappa_{5}\left(\kappa_{6}\right.$-case is similar $)$, one gets

$$
\begin{aligned}
& \left(\lambda \gamma^{r} \gamma^{m_{1} n_{1} p_{1}} \gamma^{s} \lambda\right)\left(\gamma^{r s q} \gamma^{m_{4} n_{4} p_{4}} \gamma^{r^{\prime \prime} s^{\prime \prime} q^{\prime \prime}} \gamma^{m_{5} n_{5} p_{5}}\right)^{\alpha_{5} \kappa_{6}} \\
& \left(\gamma^{m_{2} n_{2} p_{2}}\right)^{\kappa_{3} \kappa_{1}^{\prime}}\left(\gamma^{m_{3} n_{3} p_{3}}\right)^{\kappa_{4} \kappa_{2}^{\prime}} \delta_{\kappa_{1}^{\prime} \kappa_{2}^{\prime} \kappa_{3} \kappa_{4} \kappa_{6} \kappa_{9} \ldots \kappa_{11}}^{\alpha_{1}^{\prime} \ldots \ldots \ldots \alpha_{1}^{\prime} \alpha_{1}^{\prime \prime} \ldots \alpha_{3}^{\prime \prime}}
\end{aligned}
$$

If there is any contraction between $\left(\alpha_{1}^{\prime \prime}, \alpha_{1}^{\prime \prime}, \alpha_{3}^{\prime \prime}\right)$ and $\left(\kappa_{1}^{\prime}, \kappa_{2}^{\prime}, \kappa_{3}, \kappa_{4}\right)$, it is easy to see that the resulting expression vanishes. The remaining case is $\left(\alpha_{1}^{\prime \prime}, \alpha_{1}^{\prime \prime}, \alpha_{3}^{\prime \prime}\right) \in\left(\kappa_{6} \kappa_{9} \ldots \kappa_{11}\right)$. It leaves

$$
\left(\gamma^{m_{2} n_{2} p_{2}}\right)^{\kappa_{3} \kappa_{1}^{\prime}}\left(\gamma^{m_{3} n_{3} p_{3}}\right)^{\kappa_{4} \kappa_{2}^{\prime}} \delta_{\kappa_{1}^{\prime} \kappa_{2}^{\prime} \kappa_{3} \kappa_{4} \kappa}^{\alpha_{1}^{\prime} \ldots \ldots \ldots \alpha_{5}^{\prime}}
$$


where $\kappa$ is one of the indices in $\left(\kappa_{6}, \kappa_{9}, \ldots, \kappa_{11}\right)$. The contractions can be chosen to yield $\left(\lambda \gamma^{r^{\prime}} \gamma^{m_{3} n_{3} p_{3}} \gamma^{s^{\prime}} \lambda\right) \operatorname{Tr}\left(\gamma^{r^{\prime} s^{\prime} q^{\prime}} \gamma^{m_{2} n_{2} p_{2}}\right)$ which vanishes. The other cases of three- $\kappa$ combinations can similarly be shown to vanish.

(iv) When the indices, $\left(\alpha_{1}, \ldots, \alpha_{5}\right)$, contract with two out of $\left(\kappa_{1}, \ldots, \kappa_{6}\right)$ and three out of $\left(\kappa_{7}, \ldots, \kappa_{11}\right)$, one can narrow down to the case where $\left(\alpha_{1}^{\prime \prime}, \alpha_{2}^{\prime \prime}, \alpha_{3}^{\prime \prime}\right) \in\left(\kappa_{5}, \kappa_{6}, \kappa_{10}, \kappa_{11}\right)$ if the two $\kappa^{\prime}$ 's are $\left(\kappa_{1}, \kappa_{2}\right)$. Since that will leave $\delta_{\kappa_{1}^{\prime} \kappa_{2}^{\prime} \kappa_{3} \kappa_{4} \kappa}^{\alpha_{1}^{\prime} \ldots \ldots \alpha_{5}^{\prime}}$, this case vanishes too. The case where the two $\kappa^{\prime}$ 's are $\kappa_{1}\left(\right.$ or $\left.\kappa_{2}\right)$ and $\kappa_{3}\left(\right.$ or $\left.\kappa_{4}\right)$ yields the following (or something similar)

$$
\begin{aligned}
& \left(\lambda \gamma^{r} \gamma^{m_{1} n_{1} p_{1}}\right)^{\kappa_{2}}\left(\lambda \gamma^{s} \gamma^{m_{2} n_{2} p_{2}}\right)^{\kappa_{1}^{\prime}}\left(\gamma^{m_{3} n_{3} p_{3}}\right)^{\kappa_{4} \kappa_{2}^{\prime}} \\
& \left(\gamma^{m_{4} n_{4} p_{4}} \gamma^{r^{\prime \prime} s^{\prime \prime} q^{\prime \prime}} \gamma^{m_{5} n_{5} p_{5}}\right)^{\kappa_{5} \kappa_{6}} \delta_{\kappa_{1}^{\prime} \kappa_{2}^{\prime} \kappa_{2} \kappa_{4} \kappa_{5} \kappa_{6} \kappa_{10} \kappa_{11}}^{\alpha_{1}^{\prime} \ldots \ldots \ldots \alpha_{1}^{\prime} \alpha_{1 \prime}^{\prime \prime} \ldots \alpha^{\prime \prime}}
\end{aligned}
$$

Any contraction between indices $\left(\kappa_{1}^{\prime}, \kappa_{2}^{\prime}, \kappa_{1}, \kappa_{2}\right)$ and $\left(\alpha_{1}^{\prime \prime}, \alpha_{2}^{\prime \prime}, \alpha_{3}^{\prime \prime}\right)$ leads to zero: one is left with

$$
\left(\lambda \gamma^{r} \gamma^{m_{1} n_{1} p_{1}}\right)^{\kappa_{2}}\left(\lambda \gamma^{s} \gamma^{m_{2} n_{2} p_{2}}\right)^{\kappa_{1}^{\prime}}\left(\gamma^{m_{3} n_{3} p_{3}}\right)^{\kappa_{4} \kappa_{2}^{\prime}} \delta_{\kappa_{1}^{\prime} \kappa_{2}^{\prime} \kappa_{2} \kappa_{4} \kappa}^{\alpha_{1}^{\prime} \ldots \ldots \ldots \alpha_{5}^{\prime}}
$$

which can be contracted to a vanishing expression. The remaining cases can similarly be shown to vanish.

(v) When the indices, $\left(\alpha_{1}, \ldots, \alpha_{5}\right)$ contract with one of $\left(\kappa_{1}, \ldots, \kappa_{6}\right)$ and four of $\left(\kappa_{7}, \ldots, \kappa_{11}\right)$, the case whose vanishing is least trivial is when $\left(\alpha_{1}^{\prime \prime}, \alpha_{2}^{\prime \prime}, \alpha_{3}^{\prime \prime}\right)$ contract with $\kappa_{5}, \kappa_{6}$ and one of $\left(\kappa_{7}, \ldots, \kappa_{11}\right)$, say, $\kappa_{11}$, the case that results when $\left(\kappa_{7}, \ldots, \kappa_{10}\right) \in\left(\alpha_{1}, \ldots, \alpha_{5}\right)$. Then the following factors are available

$$
\left(\lambda \gamma^{r} \gamma^{m_{1} n_{1} p_{1}}\right)^{\kappa_{2}}\left(\gamma^{m_{2} n_{2} p_{2}} \gamma^{r^{\prime \prime} s^{\prime \prime} q^{\prime \prime}} \gamma^{m_{3} n_{3} p_{3}}\right)^{\kappa_{1}^{\prime} \kappa_{2}^{\prime}} \delta_{\kappa_{1}^{\prime} \kappa_{2}^{\prime} \kappa_{2} \ldots \kappa_{4}}^{\alpha_{1}^{\prime} \ldots \ldots \ldots \alpha_{5}^{\prime}}
$$

which vanishes.

(vi) The analysis of $\left(\alpha_{1}, \ldots, \alpha_{5}\right) \in\left(\kappa_{7}, \ldots, \kappa_{11}\right)$ goes similarly to that of $(\mathrm{v})$.

Let us examine the $\left(n_{d}, n_{d^{\prime}}, n_{d^{\prime \prime}}\right)=(6,5,4)$. In this case, eq.(90) becomes

$$
\begin{aligned}
& \epsilon^{\alpha_{1} \ldots \alpha_{5} \rho_{1} \ldots \rho_{11}} \epsilon^{\alpha_{1}^{\prime} \ldots \alpha_{5}^{\prime} \rho_{1}^{\prime} \ldots \rho_{11}^{\prime}} \epsilon^{\alpha_{1}^{\prime \prime} \ldots \alpha_{5}^{\prime \prime} \rho_{1}^{\prime \prime} \ldots \rho_{11}^{\prime \prime}} \quad \delta_{\sigma_{1} \ldots \sigma_{m-1} \sigma_{1}^{\prime} \sigma_{2}^{\prime} \ldots \sigma_{n}^{\prime} \sigma_{1}^{\prime \prime} \sigma_{2}^{\prime \prime} \ldots \sigma_{l+1}^{\prime \prime}}^{\rho_{1} \ldots \rho_{m-1} \rho_{m} \rho_{1}^{\prime} \ldots \rho^{\prime} \rho^{\prime} \rho^{\prime \prime} \ldots \rho^{\prime \prime}} \\
& \left(d_{\rho_{m+1}} \ldots d_{\rho_{11}} d_{\sigma_{1}} \ldots d_{\sigma_{m-1}}\right)\left(d_{\rho_{n+1}^{\prime}}^{\prime} \ldots d_{\rho_{11}^{\prime}}^{\prime} d_{\sigma_{1}^{\prime}}^{\prime} \ldots d_{\sigma_{n}^{\prime}}^{\prime}\right)\left(d_{\rho_{l+1}^{\prime \prime}}^{\prime \prime} \ldots d_{\rho_{11}^{\prime \prime}}^{\prime \prime} d_{\sigma_{1}^{\prime \prime}}^{\prime \prime} \ldots d_{\sigma_{l+1}^{\prime \prime}}^{\prime \prime}\right)
\end{aligned}
$$

Carrying out the $d$-integrations, (90) becomes

$$
\begin{aligned}
& \epsilon^{\alpha_{1} \ldots \alpha_{5} \rho_{1} \ldots \rho_{11}} \epsilon^{\alpha_{1}^{\prime} \ldots \alpha_{5}^{\prime} \rho_{1}^{\prime} \ldots \rho_{11}^{\prime}} \epsilon^{\alpha_{1}^{\prime \prime} \ldots \alpha_{5}^{\prime \prime} \rho_{1}^{\prime \prime} \ldots \rho_{11}^{\prime \prime}} \quad \delta_{\sigma_{1} \ldots \sigma_{m-1} \sigma_{1}^{\prime} \sigma_{2}^{\prime} \ldots \sigma_{n-1}^{\prime} \rho_{n}^{\prime \prime} \sigma_{2}^{\prime \prime} \ldots \sigma_{l+1}^{\prime \prime}}^{\rho_{1} \rho_{m-1} \rho_{m} \rho_{1}^{\prime}} \\
& \left(\epsilon_{\rho_{m+1} \ldots \rho_{11} \sigma_{1} \ldots \sigma_{m-1} \kappa_{1} \ldots \kappa_{6}}\right)\left(\epsilon_{\rho_{n+1}^{\prime} \ldots \rho_{11}^{\prime} \rho_{m} \sigma_{1}^{\prime} \ldots \sigma_{n}^{\prime} \kappa_{1}^{\prime} \ldots \kappa_{5}^{\prime}}\right)\left(\epsilon_{\rho_{l+1}^{\prime \prime} \ldots \rho_{11}^{\prime \prime} \rho_{n^{\prime}} \sigma_{1}^{\prime \prime} \ldots \sigma_{l+1}^{\prime \prime} \kappa_{1}^{\prime \prime} \ldots \kappa_{4}^{\prime \prime}}\right)(
\end{aligned}
$$

Using the Kronecker-delta in the manner indicated in (91), one can choose the $\epsilon$ contractions so as to produce

$$
\delta_{\kappa_{1} \ldots \ldots \ldots \kappa_{6}}^{\alpha_{1} \ldots \alpha_{5} \rho_{m}} \quad \delta_{\kappa_{1}^{\prime} \ldots \kappa_{5}^{\prime} \rho_{m}}^{\alpha_{1}^{\prime} \ldots \alpha_{m}^{\prime} \rho_{n}^{\prime}} \delta_{\kappa_{1}^{\prime \prime \ldots \kappa} \kappa_{4}^{\prime \prime} \rho_{n}^{\prime}}^{\alpha_{1 \prime \prime}^{\prime \prime} \ldots \alpha^{\prime \prime}}
$$


We classify the cases based on the distribution of $d^{\prime}$ 's over $\left(\bar{\lambda} \gamma^{m} r\right)(d W)(d W)(d W)(d W)$ : - $5 d \in\left(\bar{\lambda} \gamma^{m} r\right)(d W)^{4}$

$$
\begin{aligned}
& \left(d^{\prime} \gamma_{m_{1} n_{1} p_{1}} d^{\prime}\right)\left(d^{\prime} \gamma_{m_{2} n_{2} p_{2}} d\right)\left(d^{\prime} \gamma_{m_{3} n_{3} p_{3}} d^{\prime \prime}\right)\left(d^{\prime} \gamma_{m_{4} n_{4} p_{4}} d^{\prime \prime}\right)\left(d^{\prime \prime} \gamma_{m_{5} n_{5} p_{5}} d^{\prime \prime}\right) \\
& \left(d^{\prime} \gamma_{m_{1} n_{1} p_{1}} d^{\prime}\right)\left(d \gamma_{m_{2} n_{2} p_{2}} d^{\prime \prime}\right)\left(d^{\prime} \gamma_{m_{3} n_{3} p_{3}} d^{\prime \prime}\right)\left(d^{\prime} \gamma_{m_{4} n_{4} p_{4}} d^{\prime \prime}\right)\left(d^{\prime} \gamma_{m_{5} n_{5} p_{5}} d^{\prime \prime}\right) \\
& \left(d \gamma_{m_{1} n_{1} p_{1}} d^{\prime}\right)\left(d^{\prime} \gamma_{m_{2} n_{2} p_{2}} d^{\prime \prime}\right)\left(d^{\prime} \gamma_{m_{3} n_{3} p_{3}} d^{\prime \prime}\right)\left(d^{\prime} \gamma_{m_{4} n_{4} p_{4}} d^{\prime \prime}\right)\left(d^{\prime} \gamma_{m_{5} n_{5} p_{5}} d^{\prime \prime}\right)
\end{aligned}
$$

- $4 d, d^{\prime} \in\left(\bar{\lambda} \gamma^{m} r\right)(d W)^{4}$

$$
\begin{aligned}
& \left(d \gamma_{m_{1} n_{1} p_{1}} d\right)\left(d^{\prime} \gamma_{m_{2} n_{2} p_{2}} d^{\prime}\right)\left(d^{\prime} \gamma_{m_{3} n_{3} p_{3}} d^{\prime \prime}\right)\left(d^{\prime} \gamma_{m_{4} n_{4} p_{4}} d^{\prime \prime}\right)\left(d^{\prime \prime} \gamma_{m_{5} n_{5} p_{5}} d^{\prime \prime}\right) \\
& \left(d \gamma_{m_{1} n_{1} p_{1}} d\right)\left(d^{\prime} \gamma_{m_{2} n_{2} p_{2}} d^{\prime \prime}\right)\left(d^{\prime} \gamma_{m_{3} n_{3} p_{3}} d^{\prime \prime}\right)\left(d^{\prime} \gamma_{m_{4} n_{4} p_{4}} d^{\prime \prime}\right)\left(d^{\prime} \gamma_{m_{5} n_{5} p_{5}} d^{\prime \prime}\right) \\
& \left(d \gamma_{m_{1} n_{1} p_{1}} d^{\prime}\right)\left(d \gamma_{m_{2} n_{2} p_{2}} d^{\prime \prime}\right)\left(d^{\prime} \gamma_{m_{3} n_{3} p_{3}} d^{\prime}\right)\left(d^{\prime} \gamma_{m_{4} n_{4} p_{4}} d^{\prime \prime}\right)\left(d^{\prime \prime} \gamma_{m_{5} n_{5} p_{5}} d^{\prime \prime}\right) \\
& \left(d \gamma_{m_{1} n_{1} p_{1}} d^{\prime}\right)\left(d \gamma_{m_{2} n_{2} p_{2}} d^{\prime}\right)\left(d^{\prime} \gamma_{m_{3} n_{3} p_{3}} d^{\prime \prime}\right)\left(d^{\prime} \gamma_{m_{4} n_{4} p_{4}} d^{\prime \prime}\right)\left(d^{\prime \prime} \gamma_{m_{5} n_{5} p_{5}} d^{\prime \prime}\right) \\
& \left(d \gamma_{m_{1} n_{1} p_{1}} d^{\prime}\right)\left(d \gamma_{m_{2} n_{2} p_{2}} d^{\prime \prime}\right)\left(d^{\prime} \gamma_{m_{3} n_{3} p_{3}} d^{\prime \prime}\right)\left(d^{\prime} \gamma_{m_{4} n_{4} p_{4}} d^{\prime \prime}\right)\left(d^{\prime} \gamma_{m_{5} n_{5} p_{5}} d^{\prime \prime}\right)
\end{aligned}
$$

- $4 d, d^{\prime \prime} \in\left(\bar{\lambda} \gamma^{m} r\right)(d W)^{4}$

$$
\begin{aligned}
& \left(d \gamma_{m_{1} n_{1} p_{1}} d\right)\left(d^{\prime} \gamma_{m_{2} n_{2} p_{2}} d^{\prime}\right)\left(d^{\prime} \gamma_{m_{3} n_{3} p_{3}} d^{\prime \prime}\right)\left(d^{\prime} \gamma_{m_{4} n_{4} p_{4}} d^{\prime \prime}\right)\left(d^{\prime} \gamma_{m_{5} n_{5} p_{5}} d^{\prime \prime}\right) \\
& \left(d \gamma_{m_{1} n_{1} p_{1}} d^{\prime}\right)\left(d \gamma_{m_{2} n_{2} p_{2}} d^{\prime}\right)\left(d^{\prime} \gamma_{m_{3} n_{3} p_{3}} d^{\prime}\right)\left(d^{\prime} \gamma_{m_{4} n_{4} p_{4}} d^{\prime \prime}\right)\left(d^{\prime \prime} \gamma_{m_{5} n_{5} p_{5}} d^{\prime \prime}\right) \\
& \left(d \gamma_{m_{1} n_{1} p_{1}} d^{\prime}\right)\left(d \gamma_{m_{2} n_{2} p_{2}} d^{\prime}\right)\left(d^{\prime} \gamma_{m_{3} n_{3} p_{3}} d^{\prime \prime}\right)\left(d^{\prime} \gamma_{m_{4} n_{4} p_{4}} d^{\prime \prime}\right)\left(d^{\prime} \gamma_{m_{5} n_{5} p_{5}} d^{\prime \prime}\right)
\end{aligned}
$$

- $3 d, 2 d^{\prime} \in\left(\bar{\lambda} \gamma^{m} r\right)(d W)^{4}$

$$
\begin{aligned}
& \left(d \gamma_{m_{1} n_{1} p_{1}} d\right)\left(d \gamma_{m_{2} n_{2} p_{2}} d^{\prime \prime}\right)\left(d^{\prime} \gamma_{m_{3} n_{3} p_{3}} d^{\prime}\right)\left(d^{\prime} \gamma_{m_{4} n_{4} p_{4}} d^{\prime \prime}\right)\left(d^{\prime \prime} \gamma_{m_{5} n_{5} p_{5}} d^{\prime \prime}\right) \\
& \left(d \gamma_{m_{1} n_{1} p_{1}} d\right)\left(d \gamma_{m_{2} n_{2} p_{2}} d^{\prime}\right)\left(d^{\prime} \gamma_{m_{3} n_{3} p_{3}} d^{\prime \prime}\right)\left(d^{\prime} \gamma_{m_{4} n_{4} p_{4}} d^{\prime \prime}\right)\left(d^{\prime \prime} \gamma_{m_{5} n_{5} p_{5}} d^{\prime \prime}\right) \\
& \left(d \gamma_{m_{1} n_{1} p_{1}} d\right)\left(d \gamma_{m_{2} n_{2} p_{2}} d^{\prime \prime}\right)\left(d^{\prime} \gamma_{m_{3} n_{3} p_{3}} d^{\prime \prime}\right)\left(d^{\prime} \gamma_{m_{4} n_{4} p_{4}} d^{\prime \prime}\right)\left(d^{\prime} \gamma_{m_{5} n_{5} p_{5}} d^{\prime \prime}\right) \\
& \left(d \gamma_{m_{1} n_{1} p_{1}} d^{\prime}\right)\left(d \gamma_{m_{2} n_{2} p_{2}} d^{\prime \prime}\right)\left(d \gamma_{m_{3} n_{3} p_{3}} d^{\prime \prime}\right)\left(d^{\prime} \gamma_{m_{4} n_{4} p_{4}} d^{\prime}\right)\left(d^{\prime \prime} \gamma_{m_{5} n_{5} p_{5}} d^{\prime \prime}\right) \\
& \left(d \gamma_{m_{1} n_{1} p_{1}} d^{\prime}\right)\left(d \gamma_{m_{2} n_{2} p_{2}} d^{\prime}\right)\left(d \gamma_{m_{3} n_{3} p_{3}} d^{\prime \prime}\right)\left(d^{\prime} \gamma_{m_{4} n_{4} p_{4}} d^{\prime \prime}\right)\left(d^{\prime \prime} \gamma_{m_{5} n_{5} p_{5}} d^{\prime \prime}\right) \\
& \left(d \gamma_{m_{1} n_{1} p_{1}} d^{\prime}\right)\left(d \gamma_{m_{2} n_{2} p_{2}} d^{\prime \prime}\right)\left(d \gamma_{m_{3} n_{3} p_{3}} d^{\prime \prime}\right)\left(d^{\prime} \gamma_{m_{4} n_{4} p_{4}} d^{\prime \prime}\right)\left(d^{\prime} \gamma_{m_{5} n_{5} p_{5}} d^{\prime \prime}\right)
\end{aligned}
$$

- $3 d, d^{\prime}, d^{\prime \prime} \in\left(\bar{\lambda} \gamma^{m} r\right)(d W)^{4}$

$$
\begin{aligned}
& \left(d \gamma_{m_{1} n_{1} p_{1}} d\right)\left(d^{\prime} \gamma_{m_{2} n_{2} p_{2}} d^{\prime}\right)\left(d \gamma_{m_{3} n_{3} p_{3}} d^{\prime}\right)\left(d^{\prime} \gamma_{m_{4} n_{4} p_{4}} d^{\prime \prime}\right)\left(d^{\prime \prime} \gamma_{m_{5} n_{5} p_{5}} d^{\prime \prime}\right) \\
& \left(d \gamma_{m_{1} n_{1} p_{1}} d\right)\left(d^{\prime} \gamma_{m_{2} n_{2} p_{2}} d^{\prime}\right)\left(d^{\prime} \gamma_{m_{3} n_{3} p_{3}} d^{\prime \prime}\right)\left(d^{\prime} \gamma_{m_{4} n_{4} p_{4}} d^{\prime \prime}\right)\left(d \gamma_{m_{5} n_{5} p_{5}} d^{\prime \prime}\right) \\
& \left(d \gamma_{m_{1} n_{1} p_{1}} d\right)\left(d \gamma_{m_{2} n_{2} p_{2}} d^{\prime}\right)\left(d^{\prime} \gamma_{m_{3} n_{3} p_{3}} d^{\prime \prime}\right)\left(d^{\prime} \gamma_{m_{4} n_{4} p_{4}} d^{\prime \prime}\right)\left(d^{\prime} \gamma_{m_{5} n_{5} p_{5}} d^{\prime \prime}\right) \\
& \left(d \gamma_{m_{1} n_{1} p_{1}} d^{\prime}\right)\left(d \gamma_{m_{2} n_{2} p_{2}} d^{\prime}\right)\left(d \gamma_{m_{3} n_{3} p_{3}} d^{\prime \prime}\right)\left(d^{\prime} \gamma_{m_{4} n_{4} p_{4}} d^{\prime}\right)\left(d^{\prime \prime} \gamma_{m_{5} n_{5} p_{5}} d^{\prime \prime}\right) \\
& \left(d \gamma_{m_{1} n_{1} p_{1}} d^{\prime}\right)\left(d \gamma_{m_{2} n_{2} p_{2}} d^{\prime}\right)\left(d \gamma_{m_{3} n_{3} p_{3}} d^{\prime}\right)\left(d^{\prime} \gamma_{m_{4} n_{4} p_{4}} d^{\prime \prime}\right)\left(d^{\prime \prime} \gamma_{m_{5} n_{5} p_{5}} d^{\prime \prime}\right) \\
& \left(d \gamma_{m_{1} n_{1} p_{1}} d^{\prime}\right)\left(d \gamma_{m_{2} n_{2} p_{2}} d^{\prime}\right)\left(d \gamma_{m_{3} n_{3} p_{3}} d^{\prime \prime}\right)\left(d^{\prime} \gamma_{m_{4} n_{4} p_{4}} d^{\prime \prime}\right)\left(d^{\prime} \gamma_{m_{5} n_{5} p_{5}} d^{\prime \prime}\right)
\end{aligned}
$$

- $3 d, 2 d^{\prime \prime} \in\left(\bar{\lambda} \gamma^{m} r\right)(d W)^{4}$

$$
\begin{aligned}
& \left(d \gamma_{m_{1} n_{1} p_{1}} d\right)\left(d \gamma_{m_{2} n_{2} p_{2}} d^{\prime}\right)\left(d^{\prime} \gamma_{m_{3} n_{3} p_{3}} d^{\prime}\right)\left(d^{\prime} \gamma_{m_{4} n_{4} p_{4}} d^{\prime \prime}\right)\left(d^{\prime} \gamma_{m_{5} n_{5} p_{5}} d^{\prime \prime}\right) \\
& \left(d \gamma_{m_{1} n_{1} p_{1}} d^{\prime}\right)\left(d \gamma_{m_{2} n_{2} p_{2}} d^{\prime}\right)\left(d \gamma_{m_{3} n_{3} p_{3}} d^{\prime}\right)\left(d^{\prime} \gamma_{m_{4} n_{4} p_{4}} d^{\prime}\right)\left(d^{\prime \prime} \gamma_{m_{5} n_{5} p_{5}} d^{\prime \prime}\right) \\
& \left(d \gamma_{m_{1} n_{1} p_{1}} d^{\prime}\right)\left(d \gamma_{m_{2} n_{2} p_{2}} d^{\prime}\right)\left(d \gamma_{m_{3} n_{3} p_{3}} d^{\prime}\right)\left(d^{\prime} \gamma_{m_{4} n_{4} p_{4}} d^{\prime \prime}\right)\left(d^{\prime} \gamma_{m_{5} n_{5} p_{5}} d^{\prime \prime}\right)
\end{aligned}
$$


- $2 d, 3 d^{\prime} \in\left(\bar{\lambda} \gamma^{m} r\right)(d W)^{4}$

$$
\begin{aligned}
& \left(d \gamma_{m_{1} n_{1} p_{1}} d\right)\left(d \gamma_{m_{2} n_{2} p_{2}} d^{\prime \prime}\right)\left(d \gamma_{m_{3} n_{3} p_{3}} d^{\prime \prime}\right)\left(d^{\prime} \gamma_{m_{4} n_{4} p_{4}} d^{\prime}\right)\left(d^{\prime \prime} \gamma_{m_{5} n_{5} p_{5}} d^{\prime \prime}\right) \\
& \left(d \gamma_{m_{1} n_{1} p_{1}} d\right)\left(d \gamma_{m_{2} n_{2} p_{2}} d^{\prime}\right)\left(d \gamma_{m_{3} n_{3} p_{3}} d^{\prime \prime}\right)\left(d^{\prime} \gamma_{m_{4} n_{4} p_{4}} d^{\prime \prime}\right)\left(d^{\prime \prime} \gamma_{m_{5} n_{5} p_{5}} d^{\prime \prime}\right) \\
& \left(d \gamma_{m_{1} n_{1} p_{1}} d\right)\left(d \gamma_{m_{2} n_{2} p_{2}} d^{\prime \prime}\right)\left(d \gamma_{m_{3} n_{3} p_{3}} d^{\prime \prime}\right)\left(d^{\prime} \gamma_{m_{4} n_{4} p_{4}} d^{\prime \prime}\right)\left(d^{\prime} \gamma_{m_{5} n_{5} p_{5}} d^{\prime \prime}\right) \\
& \left(d \gamma_{m_{1} n_{1} p_{1}} d^{\prime \prime}\right)\left(d \gamma_{m_{2} n_{2} p_{2}} d^{\prime \prime}\right)\left(d \gamma_{m_{3} n_{3} p_{3}} d^{\prime \prime}\right)\left(d \gamma_{m_{4} n_{4} p_{4}} d^{\prime \prime}\right)\left(d^{\prime} \gamma_{m_{5} n_{5} p_{5}} d^{\prime}\right) \\
& \left(d \gamma_{m_{1} n_{1} p_{1}} d^{\prime}\right)\left(d \gamma_{m_{2} n_{2} p_{2}} d^{\prime}\right)\left(d \gamma_{m_{3} n_{3} p_{3}} d^{\prime \prime}\right)\left(d \gamma_{m_{4} n_{4} p_{4}} d^{\prime \prime}\right)\left(d^{\prime \prime} \gamma_{m_{5} n_{5} p_{5}} d^{\prime \prime}\right) \\
& \left(d \gamma_{m_{1} n_{1} p_{1}} d^{\prime}\right)\left(d \gamma_{m_{2} n_{2} p_{2}} d^{\prime \prime}\right)\left(d \gamma_{m_{3} n_{3} p_{3}} d^{\prime \prime}\right)\left(d \gamma_{m_{4} n_{4} p_{4}} d^{\prime \prime}\right)\left(d^{\prime} \gamma_{m_{5} n_{5} p_{5}} d^{\prime \prime}\right)
\end{aligned}
$$

- $2 d, 2 d^{\prime}, d^{\prime \prime} \in\left(\bar{\lambda} \gamma^{m} r\right)(d W)^{4}$

$$
\begin{aligned}
& \left(d \gamma_{m_{1} n_{1} p_{1}} d\right)\left(d \gamma_{m_{2} n_{2} p_{2}} d^{\prime}\right)\left(d \gamma_{m_{3} n_{3} p_{3}} d^{\prime \prime}\right)\left(d^{\prime} \gamma_{m_{4} n_{4} p_{4}} d^{\prime}\right)\left(d^{\prime \prime} \gamma_{m_{5} n_{5} p_{5}} d^{\prime \prime}\right) \\
& \left(d \gamma_{m_{1} n_{1} p_{1}} d\right)\left(d \gamma_{m_{2} n_{2} p_{2}} d^{\prime}\right)\left(d \gamma_{m_{3} n_{3} p_{3}} d^{\prime}\right)\left(d^{\prime} \gamma_{m_{4} n_{4} p_{4}} d^{\prime \prime}\right)\left(d^{\prime \prime} \gamma_{m_{5} n_{5} p_{5}} d^{\prime \prime}\right) \\
& \left(d \gamma_{m_{1} n_{1} p_{1}} d\right)\left(d \gamma_{m_{2} n_{2} p_{2}} d^{\prime}\right)\left(d \gamma_{m_{3} n_{3} p_{3}} d^{\prime \prime}\right)\left(d^{\prime} \gamma_{m_{4} n_{4} p_{4}} d^{\prime \prime}\right)\left(d^{\prime} \gamma_{m_{5} n_{5} p_{5}} d^{\prime \prime}\right) \\
& \left(d \gamma_{m_{1} n_{1} p_{1}} d^{\prime}\right)\left(d \gamma_{m_{2} n_{2} p_{2}} d^{\prime \prime}\right)\left(d \gamma_{m_{3} n_{3} p_{3}} d^{\prime \prime}\right)\left(d \gamma_{m_{4} n_{4} p_{4}} d^{\prime \prime}\right)\left(d^{\prime} \gamma_{m_{5} n_{5} p_{5}} d^{\prime}\right) \\
& \left(d \gamma_{m_{1} n_{1} p_{1}} d^{\prime}\right)\left(d \gamma_{m_{2} n_{2} p_{2}} d^{\prime}\right)\left(d \gamma_{m_{3} n_{3} p_{3}} d^{\prime}\right)\left(d \gamma_{m_{4} n_{4} p_{4}} d^{\prime \prime}\right)\left(d^{\prime \prime} \gamma_{m_{5} n_{5} p_{5}} d^{\prime \prime}\right) \\
& \left(d \gamma_{m_{1} n_{1} p_{1}} d^{\prime}\right)\left(d \gamma_{m_{2} n_{2} p_{2}} d^{\prime}\right)\left(d \gamma_{m_{3} n_{3} p_{3}} d^{\prime \prime}\right)\left(d \gamma_{m_{4} n_{4} p_{4}} d^{\prime \prime}\right)\left(d^{\prime} \gamma_{m_{5} n_{5} p_{5}} d^{\prime \prime}\right)
\end{aligned}
$$

- $2 d, d^{\prime}, 2 d^{\prime \prime} \in\left(\bar{\lambda} \gamma^{m} r\right)(d W)^{4}$

$$
\begin{aligned}
& \left(d \gamma_{m_{1} n_{1} p_{1}} d\right)\left(d \gamma_{m_{2} n_{2} p_{2}} d^{\prime}\right)\left(d \gamma_{m_{3} n_{3} p_{3}} d^{\prime}\right)\left(d^{\prime} \gamma_{m_{4} n_{4} p_{4}} d^{\prime}\right)\left(d^{\prime \prime} \gamma_{m_{5} n_{5} p_{5}} d^{\prime \prime}\right) \\
& \left(d \gamma_{m_{1} n_{1} p_{1}} d\right)\left(d \gamma_{m_{2} n_{2} p_{2}} d^{\prime}\right)\left(d \gamma_{m_{3} n_{3} p_{3}} d^{\prime \prime}\right)\left(d^{\prime} \gamma_{m_{4} n_{4} p_{4}} d^{\prime}\right)\left(d^{\prime} \gamma_{m_{5} n_{5} p_{5}} d^{\prime \prime}\right) \\
& \left(d \gamma_{m_{1} n_{1} p_{1}} d\right)\left(d \gamma_{m_{2} n_{2} p_{2}} d^{\prime}\right)\left(d \gamma_{m_{3} n_{3} p_{3}} d^{\prime}\right)\left(d^{\prime} \gamma_{m_{4} n_{4} p_{4}} d^{\prime \prime}\right)\left(d^{\prime} \gamma_{m_{5} n_{5} p_{5}} d^{\prime \prime}\right) \\
& \left(d^{\prime} \gamma_{m_{1} n_{1} p_{1}} d^{\prime}\right)\left(d \gamma_{m_{2} n_{2} p_{2}} d^{\prime}\right)\left(d \gamma_{m_{3} n_{3} p_{3}} d^{\prime}\right)\left(d \gamma_{m_{4} n_{4} p_{4}} d^{\prime \prime}\right)\left(d \gamma_{m_{5} n_{5} p_{5}} d^{\prime \prime}\right) \\
& \left(d \gamma_{m_{1} n_{1} p_{1}} d^{\prime}\right)\left(d \gamma_{m_{2} n_{2} p_{2}} d^{\prime}\right)\left(d \gamma_{m_{3} n_{3} p_{3}} d^{\prime}\right)\left(d \gamma_{m_{4} n_{4} p_{4}} d^{\prime}\right)\left(d^{\prime \prime} \gamma_{m_{5} n_{5} p_{5}} d^{\prime \prime}\right) \\
& \left(d \gamma_{m_{1} n_{1} p_{1}} d^{\prime}\right)\left(d \gamma_{m_{2} n_{2} p_{2}} d^{\prime}\right)\left(d \gamma_{m_{3} n_{3} p_{3}} d^{\prime}\right)\left(d \gamma_{m_{4} n_{4} p_{4}} d^{\prime \prime}\right)\left(d^{\prime} \gamma_{m_{5} n_{5} p_{5}} d^{\prime \prime}\right)
\end{aligned}
$$

- $2 d, 3 d^{\prime \prime} \in\left(\bar{\lambda} \gamma^{m} r\right)(d W)^{4}$

$$
\begin{aligned}
& \left(d \gamma_{m_{1} n_{1} p_{1}} d\right)\left(d^{\prime} \gamma_{m_{2} n_{2} p_{2}} d^{\prime}\right)\left(d \gamma_{m_{3} n_{3} p_{3}} d^{\prime}\right)\left(d \gamma_{m_{4} n_{4} p_{4}} d^{\prime}\right)\left(d^{\prime} \gamma_{m_{5} n_{5} p_{5}} d^{\prime \prime}\right) \\
& \left(d \gamma_{m_{1} n_{1} p_{1}} d^{\prime}\right)\left(d \gamma_{m_{2} n_{2} p_{2}} d^{\prime}\right)\left(d \gamma_{m_{3} n_{3} p_{3}} d^{\prime}\right)\left(d \gamma_{m_{4} n_{4} p_{4}} d^{\prime \prime}\right)\left(d^{\prime} \gamma_{m_{5} n_{5} p_{5}} d^{\prime}\right) \\
& \left(d \gamma_{m_{1} n_{1} p_{1}} d^{\prime}\right)\left(d \gamma_{m_{2} n_{2} p_{2}} d^{\prime}\right)\left(d \gamma_{m_{3} n_{3} p_{3}} d^{\prime}\right)\left(d \gamma_{m_{4} n_{4} p_{4}} d^{\prime}\right)\left(d^{\prime} \gamma_{m_{5} n_{5} p_{5}} d^{\prime \prime}\right)
\end{aligned}
$$

- $d, 4 d^{\prime} \in\left(\bar{\lambda} \gamma^{m} r\right)(d W)^{4}$

$$
\begin{aligned}
& \left(d \gamma_{m_{1} n_{1} p_{1}} d\right)\left(d \gamma_{m_{2} n_{2} p_{2}} d^{\prime}\right)\left(d \gamma_{m_{3} n_{3} p_{3}} d^{\prime \prime}\right)\left(d \gamma_{m_{4} n_{4} p_{4}} d^{\prime \prime}\right)\left(d^{\prime \prime} \gamma_{m_{5} n_{5} p_{5}} d^{\prime \prime}\right) \\
& \left(d \gamma_{m_{1} n_{1} p_{1}} d\right)\left(d \gamma_{m_{2} n_{2} p_{2}} d^{\prime \prime}\right)\left(d \gamma_{m_{3} n_{3} p_{3}} d^{\prime \prime}\right)\left(d \gamma_{m_{4} n_{4} p_{4}} d^{\prime \prime}\right)\left(d^{\prime} \gamma_{m_{5} n_{5} p_{5}} d^{\prime \prime}\right) \\
& \left(d \gamma_{m_{1} n_{1} p_{1}} d^{\prime}\right)\left(d \gamma_{m_{2} n_{2} p_{2}} d^{\prime \prime}\right)\left(d \gamma_{m_{3} n_{3} p_{3}} d^{\prime \prime}\right)\left(d \gamma_{m_{4} n_{4} p_{4}} d^{\prime \prime}\right)\left(d \gamma_{m_{5} n_{5} p_{5}} d^{\prime \prime}\right)
\end{aligned}
$$

- $d, 3 d^{\prime}, d^{\prime \prime} \in\left(\bar{\lambda} \gamma^{m} r\right)(d W)^{4}$

$$
\begin{aligned}
& \left(d \gamma_{m_{1} n_{1} p_{1}} d\right)\left(d \gamma_{m_{2} n_{2} p_{2}} d^{\prime \prime}\right)\left(d \gamma_{m_{3} n_{3} p_{3}} d^{\prime \prime}\right)\left(d \gamma_{m_{4} n_{4} p_{4}} d^{\prime \prime}\right)\left(d^{\prime} \gamma_{m_{5} n_{5} p_{5}} d^{\prime}\right) \\
& \left(d \gamma_{m_{1} n_{1} p_{1}} d\right)\left(d \gamma_{m_{2} n_{2} p_{2}} d^{\prime}\right)\left(d \gamma_{m_{3} n_{3} p_{3}} d^{\prime}\right)\left(d \gamma_{m_{4} n_{4} p_{4}} d^{\prime \prime}\right)\left(d^{\prime \prime} \gamma_{m_{5} n_{5} p_{5}} d^{\prime \prime}\right) \\
& \left(d \gamma_{m_{1} n_{1} p_{1}} d\right)\left(d \gamma_{m_{2} n_{2} p_{2}} d^{\prime}\right)\left(d \gamma_{m_{3} n_{3} p_{3}} d^{\prime \prime}\right)\left(d \gamma_{m_{4} n_{4} p_{4}} d^{\prime \prime}\right)\left(d^{\prime} \gamma_{m_{5} n_{5} p_{5}} d^{\prime \prime}\right) \\
& \left(d \gamma_{m_{1} n_{1} p_{1}} d^{\prime}\right)\left(d \gamma_{m_{2} n_{2} p_{2}} d^{\prime}\right)\left(d \gamma_{m_{3} n_{3} p_{3}} d^{\prime \prime}\right)\left(d \gamma_{m_{4} n_{4} p_{4}} d^{\prime \prime}\right)\left(d \gamma_{m_{5} n_{5} p_{5}} d^{\prime \prime}\right)
\end{aligned}
$$


- $d, 2 d^{\prime}, 2 d^{\prime \prime} \in\left(\bar{\lambda} \gamma^{m} r\right)(d W)^{4}$

$$
\begin{aligned}
& \left(d \gamma_{m_{1} n_{1} p_{1}} d\right)\left(d \gamma_{m_{2} n_{2} p_{2}} d^{\prime}\right)\left(d \gamma_{m_{3} n_{3} p_{3}} d^{\prime \prime}\right)\left(d \gamma_{m_{4} n_{4} p_{4}} d^{\prime \prime}\right)\left(d^{\prime} \gamma_{m_{5} n_{5} p_{5}} d^{\prime}\right) \\
& \left(d \gamma_{m_{1} n_{1} p_{1}} d\right)\left(d \gamma_{m_{2} n_{2} p_{2}} d^{\prime}\right)\left(d \gamma_{m_{3} n_{3} p_{3}} d^{\prime}\right)\left(d \gamma_{m_{4} n_{4} p_{4}} d^{\prime}\right)\left(d^{\prime \prime} \gamma_{m_{5} n_{5} p_{5}} d^{\prime \prime}\right) \\
& \left(d \gamma_{m_{1} n_{1} p_{1}} d^{\prime}\right)\left(d \gamma_{m_{2} n_{2} p_{2}} d^{\prime}\right)\left(d \gamma_{m_{3} n_{3} p_{3}} d^{\prime}\right)\left(d \gamma_{m_{4} n_{4} p_{4}} d^{\prime \prime}\right)\left(d \gamma_{m_{5} n_{5} p_{5}} d^{\prime \prime}\right)
\end{aligned}
$$

- $d, d^{\prime}, 3 d^{\prime \prime} \in\left(\bar{\lambda} \gamma^{m} r\right)(d W)^{4}$

$$
\begin{aligned}
& \left(d \gamma_{m_{1} n_{1} p_{1}} d\right)\left(d \gamma_{m_{2} n_{2} p_{2}} d^{\prime}\right)\left(d \gamma_{m_{3} n_{3} p_{3}} d^{\prime}\right)\left(d \gamma_{m_{4} n_{4} p_{4}} d^{\prime \prime}\right)\left(d^{\prime} \gamma_{m_{5} n_{5} p_{5}} d^{\prime}\right) \\
& \left(d \gamma_{m_{1} n_{1} p_{1}} d\right)\left(d \gamma_{m_{2} n_{2} p_{2}} d^{\prime}\right)\left(d \gamma_{m_{3} n_{3} p_{3}} d^{\prime}\right)\left(d \gamma_{m_{4} n_{4} p_{4}} d^{\prime}\right)\left(d^{\prime} \gamma_{m_{5} n_{5} p_{5}} d^{\prime \prime}\right) \\
& \left(d \gamma_{m_{1} n_{1} p_{1}} d^{\prime}\right)\left(d \gamma_{m_{2} n_{2} p_{2}} d^{\prime}\right)\left(d \gamma_{m_{3} n_{3} p_{3}} d^{\prime}\right)\left(d \gamma_{m_{4} n_{4} p_{4}} d^{\prime}\right)\left(d \gamma_{m_{5} n_{5} p_{5}} d^{\prime \prime}\right)
\end{aligned}
$$

- $d, 4 d^{\prime \prime} \in\left(\bar{\lambda} \gamma^{m} r\right)(d W)^{4}$

$$
\begin{aligned}
& \left(d \gamma_{m_{1} n_{1} p_{1}} d\right)\left(d \gamma_{m_{2} n_{2} p_{2}} d^{\prime}\right)\left(d \gamma_{m_{3} n_{3} p_{3}} d^{\prime}\right)\left(d \gamma_{m_{4} n_{4} p_{4}} d^{\prime}\right)\left(d^{\prime} \gamma_{m_{5} n_{5} p_{5}} d^{\prime}\right) \\
& \left(d \gamma_{m_{1} n_{1} p_{1}} d^{\prime}\right)\left(d \gamma_{m_{2} n_{2} p_{2}} d^{\prime}\right)\left(d \gamma_{m_{3} n_{3} p_{3}} d^{\prime}\right)\left(d \gamma_{m_{4} n_{4} p_{4}} d^{\prime}\right)\left(d \gamma_{m_{5} n_{5} p_{5}} d^{\prime}\right)
\end{aligned}
$$

- $5 d^{\prime} \in\left(\bar{\lambda} \gamma^{m} r\right)(d W)^{4}$

$$
\left(d \gamma_{m_{1} n_{1} p_{1}} d\right)\left(d \gamma_{m_{2} n_{2} p_{2}} d^{\prime \prime}\right)\left(d \gamma_{m_{3} n_{3} p_{3}} d^{\prime \prime}\right)\left(d \gamma_{m_{4} n_{4} p_{4}} d^{\prime \prime}\right)\left(d \gamma_{m_{5} n_{5} p_{5}} d^{\prime \prime}\right)
$$

- $4 d^{\prime}, d^{\prime \prime} \in\left(\bar{\lambda} \gamma^{m} r\right)(d W)^{4}$

$$
\left(d \gamma_{m_{1} n_{1} p_{1}} d\right)\left(d \gamma_{m_{2} n_{2} p_{2}} d^{\prime}\right)\left(d \gamma_{m_{3} n_{3} p_{3}} d^{\prime \prime}\right)\left(d \gamma_{m_{4} n_{4} p_{4}} d^{\prime \prime}\right)\left(d \gamma_{m_{5} n_{5} p_{5}} d^{\prime \prime}\right)
$$

- $3 d^{\prime}, 2 d^{\prime \prime} \in\left(\bar{\lambda} \gamma^{m} r\right)(d W)^{4}$

$$
\left(d \gamma_{m_{1} n_{1} p_{1}} d\right)\left(d \gamma_{m_{2} n_{2} p_{2}} d^{\prime}\right)\left(d \gamma_{m_{3} n_{3} p_{3}} d^{\prime}\right)\left(d \gamma_{m_{4} n_{4} p_{4}} d^{\prime \prime}\right)\left(d \gamma_{m_{5} n_{5} p_{5}} d^{\prime \prime}\right)
$$

- $2 d^{\prime}, 3 d^{\prime \prime} \in\left(\bar{\lambda} \gamma^{m} r\right)(d W)^{4}$

$$
\left(d \gamma_{m_{1} n_{1} p_{1}} d\right)\left(d \gamma_{m_{2} n_{2} p_{2}} d^{\prime}\right)\left(d \gamma_{m_{3} n_{3} p_{3}} d^{\prime}\right)\left(d \gamma_{m_{4} n_{4} p_{4}} d^{\prime}\right)\left(d \gamma_{m_{5} n_{5} p_{5}} d^{\prime \prime}\right)
$$

- $d^{\prime}, 4 d^{\prime \prime} \in\left(\bar{\lambda} \gamma^{m} r\right)(d W)^{4}$

$$
\left(d \gamma_{m_{1} n_{1} p_{1}} d\right)\left(d \gamma_{m_{2} n_{2} p_{2}} d^{\prime}\right)\left(d \gamma_{m_{3} n_{3} p_{3}} d^{\prime}\right)\left(d \gamma_{m_{4} n_{4} p_{4}} d^{\prime}\right)\left(d \gamma_{m_{5} n_{5} p_{5}} d^{\prime}\right)
$$

We have used short-hand notations. For example, the case where five $d$ 's of the six $d$ 's ( six since $n_{d}=6$ ) come from $\left(\bar{\lambda} \gamma^{m} r\right)(d W)(d W)(d W)(d W)$ has been denoted by

$$
5 d^{\prime} s \in\left(\bar{\lambda} \gamma^{m} r\right)(d W)(d W)(d W)(d W)
$$

There are multiple ways to show that each of these terms vanishes. We illustrate the computations with a few examples. Our first example is the first term in (C.32),

$$
\left(d^{\prime} \gamma_{m_{1} n_{1} p_{1}} d^{\prime}\right)\left(d^{\prime} \gamma_{m_{2} n_{2} p_{2}} d\right)\left(d^{\prime} \gamma_{m_{3} n_{3} p_{3}} d^{\prime \prime}\right)\left(d^{\prime} \gamma_{m_{4} n_{4} p_{4}} d^{\prime \prime}\right)\left(d^{\prime \prime} \gamma_{m_{5} n_{5} p_{5}} d^{\prime \prime}\right)
$$


The $d$-integration yields

$$
\left(\gamma_{m_{1} n_{1} p_{1}}\right)^{\kappa_{1}^{\prime} \kappa_{2}^{\prime}}\left(\gamma_{m_{2} n_{2} p_{2}}\right)^{\kappa_{3}^{\prime} \kappa_{1}}\left(\gamma_{m_{3} n_{3} p_{3}}\right)^{\kappa_{4}^{\prime} \kappa_{1}^{\prime \prime}}\left(\gamma_{m_{4} n_{4} p_{4}}\right)^{\kappa_{5}^{\prime} \kappa_{2}^{\prime \prime}}\left(\gamma_{m_{5} n_{5} p_{5}}\right)^{\kappa_{3}^{\prime \prime} \kappa_{4}^{\prime \prime}}
$$

The index contractions dictated by the third factor of Kronecker-deltas in (C.31) can be chosen as

$$
\alpha_{1}^{\prime \prime}=\kappa_{1}^{\prime \prime}, \quad \alpha_{2}^{\prime \prime}=\kappa_{2}^{\prime \prime}, \quad \alpha_{3}^{\prime \prime}=\rho_{n}^{\prime}, \quad \alpha_{4}^{\prime \prime}=\kappa_{3}^{\prime \prime}, \quad \alpha_{5}^{\prime \prime}=\kappa_{4}^{\prime \prime}
$$

It leads to

$$
\left(\gamma^{m_{3} n_{3} p_{3}} \gamma^{r^{\prime \prime}} \lambda\right)^{\kappa_{4}^{\prime}}\left(\gamma^{m_{4} n_{4} p_{4}} \gamma^{s^{\prime \prime}} \lambda\right)^{\kappa_{5}^{\prime}} \operatorname{Tr}\left(\gamma^{r^{\prime \prime} s^{\prime \prime} q^{\prime \prime}} \gamma^{m_{5} n_{5} p_{5}}\right) \delta_{\kappa_{1}^{\prime} \ldots \kappa_{5}^{\prime} \rho_{m}}^{\alpha_{1}^{\prime} \ldots \alpha_{2}^{\prime} \alpha^{\prime \prime}}
$$

which always reduces to an vanishing expression. Our second example is the sixth term in (C.35):

$$
\left(d \gamma_{m_{1} n_{1} p_{1}} d^{\prime}\right)\left(d \gamma_{m_{2} n_{2} p_{2}} d^{\prime \prime}\right)\left(d \gamma_{m_{3} n_{3} p_{3}} d^{\prime \prime}\right)\left(d^{\prime} \gamma_{m_{4} n_{4} p_{4}} d^{\prime \prime}\right)\left(d^{\prime} \gamma_{m_{5} n_{5} p_{5}} d^{\prime \prime}\right)
$$

The $d$-integration followed by the same choice as (C.55) yields,

$$
\left(\gamma^{m_{2} n_{2} p_{2}} \gamma^{r^{\prime \prime}} \lambda\right)^{\kappa_{2}}\left(\gamma^{m_{3} n_{3} p_{3}} \gamma^{s^{\prime \prime}} \lambda\right)^{\kappa_{3}}\left(\gamma^{m_{4} n_{4} p_{4}} \gamma^{r^{\prime \prime} s^{\prime \prime} q^{\prime \prime}} \gamma^{m_{5} n_{5} p_{5}}\right)^{\kappa_{2}^{\prime} \kappa_{3}^{\prime}} \delta_{\kappa_{1} \ldots \kappa_{5} \kappa_{6}}^{\alpha_{1} \ldots \alpha_{5} \rho_{m}} \delta_{\kappa_{1}^{\prime} \ldots \kappa_{5}^{\prime} \rho_{m}}^{\alpha_{1}^{\prime} \ldots \alpha_{3}^{\prime} \alpha_{3}^{\prime \prime}}
$$

where only the relevant factors are recorded. As one can easily check, it always leads to a vanishing expression. Let us take the fourth term in (C.39), the case where $2 d, 2 d^{\prime}, d^{\prime \prime} \in\left(\bar{\lambda} \gamma^{m} r\right)(d W)^{4}$, as the third example,

$$
\left(d \gamma_{m_{1} n_{1} p_{1}} d^{\prime}\right)\left(d \gamma_{m_{2} n_{2} p_{2}} d^{\prime \prime}\right)\left(d \gamma_{m_{3} n_{3} p_{3}} d^{\prime \prime}\right)\left(d \gamma_{m_{4} n_{4} p_{4}} d^{\prime \prime}\right)\left(d^{\prime} \gamma_{m_{5} n_{5} p_{5}} d^{\prime}\right)
$$

The steps similar to those above yield,

$$
\left(\gamma^{m_{2} n_{2} p_{2}} \gamma^{r^{\prime \prime}} \lambda\right)^{\kappa_{2}}\left(\gamma^{m_{3} n_{3} p_{3}} \gamma^{r^{\prime \prime} s^{\prime \prime} q^{\prime \prime}} \gamma^{m_{4} n_{4} p_{4}}\right)^{\kappa_{3} \kappa_{4}} \delta_{\kappa_{1} \ldots \kappa_{5} \kappa_{6}}^{\alpha_{1} \ldots \alpha_{5} \rho_{m}} \delta_{\kappa_{1}^{\prime} \ldots \kappa_{5}^{\prime} \rho_{m}}^{\alpha_{1}^{\prime} \ldots \alpha_{5}^{\prime} \alpha_{3}^{\prime \prime}}
$$

It again leads to a vanishing expression. 


\section{References}

[1] J. Polchinski, "Dirichlet-Branes and Ramond-Ramond Charges," Phys. Rev. Lett. 75, 4724 (1995) [arXiv:hep-th/9510017]; "Lectures on D-branes," arXiv:hepth/9611050

[2] J. Polchinski, String theory, vol 1,2, Cambridge

[3] C. V. Johnson, "D-brane primer," arXiv:hep-th/0007170.

[4] T. Banks, W. Fischler, S. H. Shenker and L. Susskind, "M theory as a matrix model: A conjecture," Phys. Rev. D 55 (1997) 5112 [arXiv:hep-th/9610043].

[5] N. Ishibashi, H. Kawai, Y. Kitazawa and A. Tsuchiya, "A large-N reduced model as superstring," Nucl. Phys. B 498, 467 (1997) [arXiv:hep-th/9612115].

[6] J. M. Maldacena, "The large N limit of superconformal field theories and supergravity," Adv. Theor. Math. Phys. 2, 231 (1998) [Int. J. Theor. Phys. 38, 1113 (1999)] [arXiv:hep-th/9711200].

[7] S. S. Gubser, I. R. Klebanov and A. M. Polyakov, "Gauge theory correlators from non-critical string theory," Phys. Lett. B 428, 105 (1998) [arXiv:hep-th/9802109].

[8] E. Witten, "Anti-de Sitter space and holography," Adv. Theor. Math. Phys. 2, 253 (1998) [arXiv:hep-th/9802150].

[9] O. Aharony, S. S. Gubser, J. M. Maldacena, H. Ooguri and Y. Oz, "Large N field theories, string theory and gravity," Phys. Rept. 323, 183 (2000) [arXiv:hepth/9905111].

[10] H. Kawai and T. Suyama, "AdS/CFT Correspondence as a Consequence of Scale Invariance," arXiv:0706.1163 [hep-th], ; "Some Implications of Perturbative Approach to AdS/CFT Correspondence," Nucl. Phys. B 794, 1 (2008) [arXiv:0708.2463 [hep-th]].

[11] I. Y. Park, "Strong coupling limit of open strings: Born-Infeld analysis," Phys. Rev. D 64, 081901 (2001) [arXiv:hep-th/0106078].

[12] H. B. Nielsen and P. Olesen, "Local Field Theory Of The Dual String," Nucl. Phys. B 57 (1973) 367.

[13] G. W. Gibbons, K. Hori and P. Yi, "String fluid from unstable D-branes," Nucl. Phys. B 596, 136 (2001) [arXiv:hep-th/0009061].

[14] A. Sen, "Fundamental strings in open string theory at the tachyonic vacuum," J. Math. Phys. 42, 2844 (2001) [arXiv:hep-th/0010240]. 
[15] I. Y. Park, "Scattering on D3-branes," Phys. Lett. B 660, 583 (2008) [arXiv:0708.3452 [hep-th]] ; "One loop scattering on D-branes," Eur. Phys. J. C 62: 783 (2009), arXiv:0801.0218 [hep-th]

[16] I. Y. Park, "Open string engineering of D-brane geometry," JHEP 0808, 026 (2008) [arXiv:0806.3330 [hep-th]].

[17] I. Y. Park, "Geometric counter-vertex for open string scattering on D-branes," arXiv:0902.1279 [hep-th], to appear in EPJC.

[18] Z. J. Zheng, J. B. Wu and C. J. Zhu, "Two-loop superstrings in hyperelliptic language. III: The four-particle amplitude," Nucl. Phys. B 663, 95 (2003) [arXiv:hepth/0212219].

[19] E. D'Hoker and D. H. Phong, "Two-Loop Superstrings VI: Non-Renormalization Theorems and the 4-Point Function," Nucl. Phys. B 715, 3 (2005) [arXiv:hepth/0501197].

[20] N. Berkovits, "Super-Poincare covariant quantization of the superstring," JHEP 0004, 018 (2000) [arXiv:hep-th/0001035] ; "ICTP lectures on covariant quantization of the superstring," arXiv:hep-th/0209059 ; "Pure spinor formalism as an N = 2 topological string," JHEP 0510, 089 (2005) [arXiv:hep-th/0509120].

[21] W. Siegel, "Classical Superstring Mechanics," Nucl. Phys. B 263, 93 (1986).

[22] N. Berkovits and O. Chandia, "Massive superstring vertex operator in $\mathrm{D}=10$ superspace," JHEP 0208, 040 (2002) [arXiv:hep-th/0204121].

[23] N. Berkovits and V. Pershin, "Supersymmetric Born-Infeld from the pure spinor formalism of the open superstring," JHEP 0301, 023 (2003) [arXiv:hepth/0205154].

[24] N. Berkovits and B. C. Vallilo, "Consistency of super-Poincare covariant superstring tree amplitudes," JHEP 0007, 015 (2000) [arXiv:hep-th/0004171].

[25] N. Berkovits and C. R. Mafra, "Some superstring amplitude computations with the non-minimal pure spinor formalism," JHEP 0611, 079 (2006) [arXiv:hepth/0607187].

[26] Y. Aisaka and Y. Kazama, "A new first class algebra, homological perturbation and extension of pure spinor formalism for superstring," JHEP 0302, 017 (2003) [arXiv:hep-th/0212316] ;

[27] P. A. Grassi and P. Vanhove, "Topological M theory from pure spinor formalism," Adv. Theor. Math. Phys. 9, 285 (2005) [arXiv:hep-th/0411167] ; "Higher-loop amplitudes in the non-minimal pure spinor formalism," JHEP 0905, 089 (2009) [arXiv:0903.3903 [hep-th]]. 
[28] G. Policastro and D. Tsimpis, "R**4, purified," Class. Quant. Grav. 23, 4753 (2006) [arXiv:hep-th/0603165].

[29] V. Alexandrov, D. Krotov, A. Losev and V. Lysov, "On Pure Spinor Superfield Formalism," JHEP 0710, 074 (2007) [arXiv:0705.2191 [hep-th]].

[30] C. R. Mafra, "Superstring Scattering Amplitudes with the Pure Spinor Formalism," arXiv:0902.1552 [hep-th].

[31] S. Gerigk and I. Kirsch, "On the Relation between Hybrid and Pure Spinor String Theory," JHEP 1003, 106 (2010) [arXiv:0912.2347 [hep-th]].

[32] H. Gomez and C. R. Mafra, "The Overall Coefficient of the Two-loop Superstring Amplitude Using Pure Spinors," arXiv:1003.0678 [hep-th].

[33] H. Gomez, "One-loop Superstring Amplitude From Integrals on Pure Spinors Space," arXiv:0910.3405 [Unknown].

[34] Y. Aisaka and N. Berkovits, "Pure Spinor Vertex Operators in Siegel Gauge and Loop Amplitude Regularization," JHEP 0907, 062 (2009) [arXiv:0903.3443 [hepth]].

[35] N. Berkovits and N. Nekrasov, "Multiloop superstring amplitudes from nonminimal pure spinor formalism," JHEP 0612, 029 (2006) [arXiv:hep-th/0609012].

[36] J. Hoogeveen and K. Skenderis, "BRST quantization of the pure spinor superstring," JHEP 0711, 081 (2007) [arXiv:0710.2598 [hep-th]] ; "Decoupling of unphysical states in the minimal pure spinor formalism I," JHEP 1001 (2010) 041 [arXiv:0906.3368 [hep-th]].

[37] P. Candelas and D. J. Raine, "Spontaneous Compactification And Supersymmetry In D = 11 Supergravity," Nucl. Phys. B 248, 415 (1984). 